JOURNAL OF

SYMPLECTIC GEOMETRY

Volume 12, Number 1, 49-104, 2014

\title{
THE GROUP OF CONTACT DIFFEOMORPHISMS FOR COMPACT CONTACT MANIFOLDS
}

\author{
John Bland AND Tom Duchamp
}

\begin{abstract}
For a compact contact manifold $M^{2 n+1}$, it is shown that the anisotropic Folland-Stein function spaces $\Gamma^{s}(M), s \geq(2 n+4)$ form an algebra. The notion of anisotropic regularity is extended to define the space of $\Gamma^{s}$-contact diffeomorphisms, which is shown to be a topological group under composition and a smooth Hilbert manifold. These results are used in a subsequent paper to analyse the action of the group of contact diffeomorphisms on the space of CR structures on a compact, three-dimensional manifold.
\end{abstract}

\section{Introduction}

Contact manifolds arise naturally in complex and CR geometry. The boundary of a strongly pseudoconvex domain is a contact manifold, and more generally, any strongly pseudoconvex CR manifold is a contact manifold. In each case, the $\bar{\partial}_{b}$-operator, which may be thought to embody the tangential Cauchy-Riemann equations, is a natural operator that arises in analysis. The associated second-order operator $\square_{b}$ is anisotropic, being second order in the holomorphic tangential directions and only first order in the transverse directions. In $[\mathbf{F S}]$, Folland and Stein introduced some anisotropic function spaces, the anisotropic Sobolev spaces $\Gamma^{s}$ and the anisotropic Banach spaces $\Gamma^{s, \alpha}$ to reflect this behaviour and showed that these operators are solvable with good estimates in these spaces; we henceforth refer to these as FollandStein spaces. Since the underlying symmetry group is the group of contact diffeomorphisms, in order to study the Hilbert space of CR structures up to equivalence, it is natural to hope for a Hilbert space structure on the group of contact diffeomorphisms which also respects this anisotropy.

Smooth contact diffeomorphisms were first studied by Gray $[\mathbf{G}]$. Later, Omori $[\mathbf{O 1}$, O2] showed that the space of contact diffeomorphisms is an inverse limit Hilbert (ILH ) Lie group. Omori's analysis used standard Hodge theory based on the de Rham complex and worked within the category of 
ordinary Sobolev spaces. However, since the contact distribution is a hyperplane distribution, natural compatible geometric structures will respect this anisotropy, as will the natural function spaces. These are the Folland-Stein spaces introduced in [FS].

Our main interest in the study of the group of contact diffeomorphisms is in its use as a symmetry group. In $[\mathbf{C L}]$, Cheng and Lee studied the action of the group of contact diffeomorphisms on CR structures and avoided using anisotropic spaces by working in the Nash Moser category to construct a transverse slice to the action of smooth contact diffeomorphisms. In $[\mathbf{B}]$, we restricted our attention to the case of the standard $S^{3} \subset \mathbb{C}^{2}$ and used explicit information to construct an anisotropic Hilbert space structure on contact diffeomorphisms near the identity; and in $[\mathbf{B i}]$, Biquard used a different method to construct a local parameterization for contact diffeomorphisms near the identity in the case when the contact manifold admits a free transverse $S^{1}$ action.

The main purpose of the current paper is to provide a general construction of an anisotropic Sobolev space structure on the space of contact diffeomorphisms; this structure agrees with that constructed by ad hoc methods in $[\mathbf{B}]$ and in $[\mathbf{B i}]$. Our analysis is based on the Hodge theory of the complex developed by Rumin in $[\mathbf{R}]$, which is naturally adapted to the contact structure and based on the anisotropic Folland-Stein spaces rather than the ordinary Sobolev spaces. The Folland-Stein space structure which we introduce on the space of contact diffeomorphisms plays the role of Ebin's structure on the diffeomorphism group of a general manifold $[\mathbf{E}],[\mathbf{E M}]$. In $[\mathbf{B D 3}]$, we use the results proved here to obtain normal form theorems for CauchyRiemann structures with only finite regularity.

Before discussing further the results in this paper, we briefly consider the geometric context. One of the early motivations for studying the space of $\mathrm{CR}$ structures was a suggestion by Kuranishi. In $[\mathbf{K u}]$, he observed that if one considers a normal isolated singularity in a complex space, and considers an open ball containing that singularity, then the intersection of the boundary of the ball with the complex space inherits a "partial complex structure"; that is, a Cauchy-Riemann structure which is in addition strongly pseudoconvex. In particular, the holomorphic tangent planes, which are tangent to the boundary of the sphere define a codimension one distribution which is totally non-integrable - that is, a contact distribution. Kuranishi observed that by Hartog's theorem, the Cauchy-Riemann structure on the link completely determines the complex structure on the interior, and hence the singularity up to equivalence, and vice versa. In $[\mathbf{K u}]$, he suggested that one could replace the study of the deformation space for normal isolated singularities with the deformation space for CR structures on their link; in the first case, this is a purely algebraic theory for singular spaces, whereas in the latter case, one is studying a space of smooth structures on a smooth 
manifold. Note that in complex dimensions 3 or more (CR dimensions 5 or more), the space of structures (up to equivalence) is finite-dimensional.

In [BlEp], we introduced the notion of "stably embeddable deformations" for CR structures and completed the formal part of Kuranishi's program. By "formal", we mean at the level of power series. In [Miy1, Miy2], Miyajima introduced a new double complex to do the analysis, and completed Kuranishi's program.

In three dimensions (corresponding to complex surfaces), the situation is more subtle due to the presence of non-embeddable structures. Indeed in $[\mathbf{B}]$, we showed that the space of non-embeddable structures is an infinitedimensional Hilbert space. On the other hand, results by Kiremidjian [Kir] show that these non-embeddable structures bound compact complex manifolds on the pseudoconcave side. In the case of the three sphere, these results were substantially refined by Epstein-Henkin $[\mathbf{E H}]$ providing considerable additional information.

It is worth noting that this infinite-dimensional space of non-embeddable structures corresponds to an infinite-dimensional space of geometries. In [BD1], we showed that these correspond in a natural way to deformations of the complex structure in a neighbourhood of a standard complex line $\mathbf{P}^{1}$ in $\mathbf{P}^{2}$. (The deformed manifolds are not embedded as complex submanifolds of $\mathbf{P}^{2}$.) In $[\mathbf{A B E}]$, we showed that in turn, at least at the linearized level about the standard structure, these spaces corresponded to a family of "geometries", namely the two-dimensional projective structures on a standard complex 2-ball.

These observations show that in a natural way, the space of CR structures up to equivalence defines a family of geometries up to equivalence. It is natural to study this space in more detail. In [BD3], we use the results of the current paper for this purpose. Note that in order to obtain the Hilbert space description it is necessary to use a weighted Folland-Stein space structure. This is rather easy to see on an intuitive level; indeed, two CR manifolds will be equivalent if there is a CR mapping from one to the other; finding a CR mapping is tantamount to solving a boundary Cauchy-Riemann equation, that is a CR equation; however, it is well known that sharp estimates can only be obtained using anisotropic Sobolev spaces, and indeed this is the purpose for which Folland and Stein introduced in $[\mathbf{F S}]$ what we now refer to as the Folland-Stein spaces.

Another example of this type of result is the work of Biquard $[\mathbf{B i}]$ in which he established the Positive Frequency Conjecture of Lebrun. In this paper, Biquard uses a twistor space construction to relate the existence of a filling of a conformal structure on the 3 sphere by a selfdual/antiselfdual Einstein metric on the 4 ball to the existence of a CR structure on an associated 5 manifold with positive/negative "frequency". Adapting arguments of $[\mathbf{B}]$ to his situation, he uses the presence of a free $S^{1}$ action in his model case 
to construct a weighted Sobolev space structure on the space of contact diffeomorphisms, and then uses this weighted Sobolev space structure to analyse the space of $\mathrm{CR}$ structures up to equivalence, thereby establishing the Positive Frequency Conjecture.

The paper is structured as follows. In Section 2, we introduce the anisotropic function spaces and show that, for $s \geq(2 n+4)$, they form an algebra. We believe that the sharp result here would be that the intersection of $\Gamma^{s}$ with the space of bounded functions is an algebra for all $s$; however, the result that we have stated is sufficient for our purposes. We also extend the machinery developed by Palais $[\mathbf{P a l}]$ for doing global analysis on manifolds to the context of anisotropic function spaces. In Section 2.6, we review Rumin's complex and state the results that we will use in the analysis of the space of contact diffeomorphisms. In Section 3, we show that the operations of composition and inversion are continuous in the topology induced by the anistropic structure. In Section 4, we construct a local coordinate system for the space of diffeomorphisms and then use Rumin's estimates to show the space of contact diffeomorphisms locally forms a smooth Hilbert submanifold within the coordinate chart. Using this result, we easily prove that the space of contact diffeomorphisms is a smooth Hilbert manifold modelled on the anisotropic function spaces.

1.1. Notation. We summarize here the notation and conventions used throughout the paper.

If $A$ is a subset of a topological space $X$, then $\bar{A}$ denotes the closure of $A$ in $X$. If $A$ and $B$ are subsets of $X$, then the notation $A \Subset B$ means that $A$ is compactly contained in $B$.

If $\mathcal{F}: \mathcal{A} \rightarrow \mathcal{B}$ is a map between Banach spaces, with norms $\|\cdot\|_{\mathcal{A}}$ and $\|\cdot\|_{\mathcal{B}}$, respectively, then the expression

$$
\|\mathcal{F}(f)\|_{\mathcal{B}} \prec\|f\|_{\mathcal{A}}
$$

means that there is a constant $C>0$ such that $\|\mathcal{F}(f)\|_{\mathcal{B}} \leq C\|f\|_{\mathcal{A}}$ for all $f \in \mathcal{A}$.

We give $\mathbb{R}^{m}$ the standard inner product $(\cdot, \cdot)$, and we let $|\cdot|$ denote the corresponding norm. The symbols $(\cdot, \cdot)_{s}$ and $\|\cdot\|_{s}$, for $s=0,1, \ldots$ denote the Folland-Stein inner products and norms, respectively.

If $N$ is a smooth manifold, then $T N$ and $T^{*} N$ denote its tangent and cotangent bundles, respectively; $\Lambda^{p} N$ denotes the $p$ th exterior power of $T^{*} N$; $\Omega^{p}(N)$ denotes the space of smooth $p$-forms on $N ; \mathcal{L}_{X} \beta$ denotes the Lie derivative of the form $\beta$ with respect to the vector field $X$; and $X\lrcorner \beta$ denotes interior evaluation. If $N$ has a Riemannian metric, then $|X|$ denotes the norm of the tangent vector $X$ with respect to that metric.

The symbol $C^{r}(E), r=0,1,2, \ldots, \infty$, denotes the space of $C^{r}$-sections of a fibre bundle $E \rightarrow M$, equipped with the topology of uniform convergence 
of derivatives up to order $r$ on compact sets. Similarly, $C^{r}(M, N)$ denotes the space of $C^{r}$ maps from $M$ to $N$.

We endow $\mathbb{R}^{2 n+1}$ with the contact structure defined by the one-form

$$
\eta_{0}=d x^{2 n+1}-\sum_{j=1}^{n} x^{n+j} d x^{j},
$$

where $\left(x^{1}, \ldots, x^{n}, x^{n+1}, \ldots, x^{2 n}, x^{2 n+1}\right)$ are the standard coordinates on $\mathbb{R}^{2 n+1}$, and we let $d V_{0}$ denote the standard volume form:

$$
d V_{0}=\frac{1}{n !} \eta_{0} \wedge\left(d \eta_{0}\right)^{n}
$$

We denote the contact distribution of $\eta_{0}$ by $H_{0} \subset T \mathbb{R}^{2 n+1}$, and we set

$$
\begin{gathered}
T_{0}=\frac{\partial}{\partial x^{2 n+1}}, \quad X_{j}=\frac{\partial}{\partial x^{j}}+x^{n+j} \frac{\partial}{\partial x^{2 n+1}}, \quad \text { and } \\
X_{n+j}=\frac{\partial}{\partial x^{n+j}}, \quad 1 \leq j \leq n .
\end{gathered}
$$

Observe that the collection $\left\{X_{j}, 1 \leq j \leq 2 n\right\}$ is a global framing for $H_{0}$. Note that the one-forms

$$
\eta_{0}, d x^{j}, d x^{n+j}, \quad 1 \leq j \leq n,
$$

are the dual coframe to $T_{0}, X_{j}, X_{n+j}, 1 \leq j \leq n$.

Let $f=\left(f^{1}, \ldots, f^{m}\right)$ be a smooth, $\mathbb{R}^{m}$-valued function defined on the closure of a domain $D \Subset \mathbb{R}^{2 n+1}$. We define

$$
X_{I} f= \begin{cases}X_{i_{1}} X_{i_{2}} \ldots X_{i_{t}} f, & \text { for } t>0, \\ f, & \text { for } t=0,\end{cases}
$$

where we have introduced the multi-index notation $I=\left(i_{1}, \ldots, i_{t}\right), 1 \leq i_{j} \leq$ $2 n$ and $X_{I} f=\left(X_{I} f^{1}, \ldots, X_{I} f^{m}\right)$. (For $t=0, I$ denotes the empty index $I=()$.) The integer $t$ is called the order of $I$ and written $|I|$.

Throughout this paper, $M$ denotes a fixed smooth, compact contact manifold of dimension $2 n+1$, with contact distribution $H \subset T M$. We call sections of $H$ horizontal vector fields. We let

$$
\pi_{H}: T^{*} M \rightarrow H^{*}
$$

denote the projection map; by abuse of notation, we also let

$$
\pi_{H}: \Lambda^{p} M \rightarrow \Lambda^{p} H^{*}
$$

denote the extension of $\pi_{H}$ to the exterior product bundles. For convenience, we assume that $M$ supports a fixed contact one-form ${ }^{1} \eta$. The characteristic (or Reeb) vector field $T$ is the unique vector field satisfying the conditions

\footnotetext{
${ }^{1}$ None of our results depend on this assumption, for if the line bundle $T M / H$ is nontrivial, we can lift to a double cover of $M$, where a global contact form does exist.
} 
$T\lrcorner \eta=1$ and $T\lrcorner d \eta=0$. We can then identify the dual contact distribution with the annihilator of $T$, i.e.,

$$
\left.H^{*}=\left\{\beta \in T^{*} M: T\right\lrcorner \beta=0\right\} \subset T^{*} M ;
$$

more generally

$$
\left.\Lambda^{p} H^{*}=\left\{\beta \in \Lambda^{p}(M): T\right\lrcorner \beta=0\right\},
$$

and we have the identity

$$
\left.\pi_{H}(\beta)=T\right\lrcorner(\eta \wedge \beta) .
$$

Two forms $\beta_{1}$ and $\beta_{2}$ on $M$ are said to be equal $\bmod \eta$, written

$$
\beta_{1}=\beta_{2} \bmod \eta,
$$

if and only if $\beta_{1}=\beta_{2}+\eta \wedge \alpha$, for some $\alpha \in \Omega^{*}(M)$. An easy exercise in the exterior calculus proves the equivalences

$$
\begin{gathered}
\beta_{1}=\beta_{2} \bmod \eta \Longleftrightarrow \eta \wedge\left(\beta_{1}-\beta_{2}\right)=0 \Longleftrightarrow \\
T\lrcorner\left(\left(\beta_{1}-\beta_{2}\right) \wedge \eta\right)=0 \Longleftrightarrow \pi_{H}\left(\beta_{1}\right)=\pi_{H}\left(\beta_{2}\right) .
\end{gathered}
$$

We will also fix an endomorphism $J: H \rightarrow H$ such that $J^{2}=-I d$ and such that the operator $X \mapsto d \eta(X, J X)$ is non-negative. (Such an endomorphism always exists.) We let $g$ denote the Riemannian metric defined by the formula

$$
g(X, Y)=\eta(X) \eta(Y)+d \eta(X, J Y)
$$

were we have extended $J$ to a map $J: T M \rightarrow T M$ by setting $J(T)=$ 0 . The endomorphism $J$ and the metric $g$ are said to be adapted to the contact structure. Finally, $*$ denotes the Hodge star operator associated to the metric $g$.

We say that a chart $\phi: U \rightarrow \mathbb{R}^{2 n+1}$ for $M$ is an adapted coordinate chart if $\eta=\phi^{*} \eta_{0}$. It follows that the identities $\phi_{*} T=T_{0}$ and $\phi_{*} H=H_{0}$ hold for $\phi$ adapted. An adapted atlas for $M$ is a finite, smooth atlas $\left\{\phi_{\alpha}: U_{\alpha} \rightarrow\right.$ $\left.\mathbb{R}^{2 n+1}\right\}$, consisting of adapted coordinate charts, together with open regions $D_{\alpha} \Subset \phi_{\alpha}\left(U_{\alpha}\right)$ such that $\left\{W_{\alpha}=\phi_{\alpha}^{-1}\left(D_{\alpha}\right)\right\}$ covers $M$. By compactness of $M$ and Darboux's Theorem for contact structures [Arn, p. 362], $M$ has an adapted atlas. An adapted coordinate chart for a fibre bundle $\pi: E \rightarrow M$ with $m$-dimensional fibres is a coordinate chart for $E$ of the form

$$
\psi: U \rightarrow \phi(V) \times \mathbb{R}^{m}: q \mapsto(\phi(\pi(q)), \chi(q))
$$

with $\psi$ surjective and where $\phi: V \rightarrow \mathbb{R}^{2 n+1}$ is an adapted chart for $M$. The chart is said to be centred at the point $q_{0}$ if in addition $\psi\left(q_{0}\right)=(0,0)$. If $\sigma: V \rightarrow U$ is a local section of $E$, the function $f_{\sigma}: \phi(V) \rightarrow \mathbb{R}^{m}$ defined by the formula

$$
f_{\sigma}=\chi \circ \sigma \circ \phi^{-1}
$$

is called the local representation of $\sigma$. 


\section{Global analysis on contact manifolds}

In this section, we develop some of the analytical machinery we need to study the space of contact diffeomorphisms of $M$. We begin by introducing the Folland-Stein spaces $\Gamma^{s}$ associated to a compact contact manifold. We then introduce the notions of horizontal jet of a section of a fibre bundle and define the notion of contact order of a differential operator. We close this section with a discussion of Rumin's Complex $[\mathbf{R}]$ and its associated Hodge theory, which we need in Section 4 to construct a local parameterization of the group of $\Gamma^{s}$-contact diffeomorphisms of a compact contact manifold $M$. Most of the results in this section are extensions of definitions and theorems in $[\mathbf{P a l}]$ and $[\mathbf{E}]$ to the context of contact manifolds.

2.1. Folland-Stein function spaces. Let $D$ be a bounded domain in $\mathbb{R}^{2 n+1}$. The Folland-Stein space $\Gamma^{s}\left(D, \mathbb{R}^{m}\right)$ is the Hilbert space completion of the set of smooth, $\mathbb{R}^{m}$-valued functions on $\bar{D}$ (the closure of $D$ ) with respect to the inner product

$$
(f, g)_{D, s}:=\sum_{0 \leq|I| \leq s} \int_{D}\left(X_{I} f X_{I} g\right) d V_{0} .
$$

The associated norm is written $\|f\|_{D, s}=\sqrt{(f, f)_{D, s}}$. When no confusion is likely to arise, we suppress reference to $D$ and write $\|f\|_{s}$; and we set $\Gamma^{s}(D):=\Gamma^{s}\left(D, \mathbb{R}^{1}\right)$.

Remark 2.1.1. Although we used the contact framing $\left\{X_{j}: 1 \leq j \leq 2 n\right\}$ of $H_{0}$ and the volume form $d V_{0}=\frac{1}{n !} \eta_{0} \wedge\left(d \eta_{0}\right)^{n}$ to define the inner product, an equivalent norm results if the framing is replaced by any smooth framing of $H_{0}$ and $d V_{0}$ is replaced by any smooth volume form on $\bar{D}$. In particular, suppose that $D^{\prime} \subset \mathbb{R}^{2 n+1}$ is another bounded domain and $F: \bar{D} \rightarrow \bar{D}^{\prime}$ is a smooth diffeomorphism that restricts to a contact diffeomorphism between $D$ and $D^{\prime}$. Then composition with $F$ induces an isomorphism

$$
\Gamma_{F}: \begin{cases}\Gamma^{s}\left(D^{\prime}, \mathbb{R}^{m}\right) & \rightarrow \Gamma^{s}\left(D, \mathbb{R}^{m}\right), \\ f & \mapsto f \circ F,\end{cases}
$$

between Banach spaces. To see this, note that because the derivative of $F$ respects the contact distribution, $X_{I}(f \circ F)$ is a linear combination of terms of the form $c_{J} \cdot\left(X_{J} f\right) \circ F,|J| \leq s$, where $c_{J}$ denotes a smooth function formed from $F$ and its derivatives. It follows that $f \circ F$ is of class $\Gamma^{s}$. We caution the reader that the condition that $F$ be a contact diffeomorphism is essential. For if $F$ does not preserve the contact distribution then the expansion of $X_{I}(f \circ F)$ will in general involve terms of the form $X_{J} f$ with $|J|>s$, which may not be square integrable.

The next lemma follows immediately from the definition of $\Gamma^{s}$. 
Lemma 2.1.2. The estimate

$$
\|f\|_{s} \prec \sum_{j}\left\|X_{j} f\right\|_{s-1}+\|f\|_{0}
$$

is satisfied for all $f \in \Gamma^{s}(D), s>0$.

We shall repeatedly make use of the following Sobolev Lemma for FollandStein spaces, which is an immediate corollary of [FS, Theorem 21.1].

Lemma 2.1.3. Let $D^{\prime} \Subset D \Subset \mathbb{R}^{2 n+1}$, and let $s=k+n+2, k \geq 0$. Let $f \in \Gamma^{s}\left(D, \mathbb{R}^{m}\right)$. Then the functions $X_{I} f$ are continuous on $\bar{D}^{\prime}$ for all multi-indices of order $|I| \leq k$. Moreover,

$$
\max _{x \in \bar{D}^{\prime}}\left|X_{I} f(x)\right| \prec\|f\|_{D, s}
$$

for all $|I| \leq k$. If $\|f\|_{D, s}<\infty$ for $s=2 k+n+2$, then $f$ is of class $C^{k}$ on $\bar{D}^{\prime}$. Moreover, the linear map

$$
\Gamma^{s}\left(D, \mathbb{R}^{m}\right) \rightarrow C^{k}\left(\bar{D}^{\prime}, \mathbb{R}^{m}\right)
$$

defined by restriction to $\bar{D}^{\prime}$ is continuous.

2.2. Estimates for algebraic operations. In this section, we prove some basic estimates. Lemmas 2.2.1 and 2.2.2 are needed for our proof in Section 3 that composition and inverses of contact diffeomorphisms are continuous operations. Lemma 2.2.3 and Proposition 2.3.5 are fundamental estimates used throughout the paper.

Lemma 2.2.1. Let $s \geq 2 n+3, k \leq s$ and consider open sets $D^{\prime} \Subset D \Subset$ $\mathbb{R}^{2 n+1}$. Then, for any functions $f \in \Gamma^{s}(D), g \in \Gamma^{k}(D)$,

$$
\|f \cdot g\|_{D^{\prime}, k} \prec\|f\|_{D, s} \cdot\|g\|_{D, k} .
$$

Consequently, multiplication extends to a smooth bilinear mapping

$$
\Gamma^{s}(D) \times \Gamma^{k}(D) \rightarrow \Gamma^{k}\left(D^{\prime}\right) .
$$

Proof. We need only to prove the estimate for smooth functions $f$ and $g$ on $D$. Recall that

$$
\|f \cdot g\|_{D^{\prime}, k}^{2}=\sum_{|I| \leq k} \int_{D^{\prime}}\left|X_{I}(f \cdot g)\right|^{2} d V_{0} .
$$

Applying the Leibniz rule, we find that

$$
\|f \cdot g\|_{D^{\prime}, k}^{2} \prec \sum_{|J|+|K| \leq k} \int_{D^{\prime}}\left|X_{J}(f)\right|^{2}\left|X_{K}(g)\right|^{2} d V_{0} .
$$


There are two cases to consider: $k \leq(n+1)$ and $k>n+1$. In the first case, $|J| \leq n+1$ for every summand, and we have the estimates

$$
\begin{aligned}
\int_{D^{\prime}}\left|X_{J} f\right|^{2}\left|X_{K} g\right|^{2} d V_{0} & \prec\left(\sup _{x \in D^{\prime}}\left|X_{J} f(x)\right|^{2}\right)\left\|X_{K} g\right\|_{D^{\prime}, 0}^{2} \\
& \prec\|f\|_{D,|J|+n+2}^{2}\|g\|_{D, k}^{2} \\
& \prec\|f\|_{D, s}^{2}\|g\|_{D, k}^{2},
\end{aligned}
$$

where we have used the Sobolev inequality ${ }^{2}$ (Lemma 2.1.3) at the penultimate inequality.

In the latter case, in each term either $|J| \leq n+1$ or $|K| \leq k-(n+2)$. In the first instance, we bound the term by $\|f\|_{D, s}^{2}\|g\|_{D, k}^{2}$ as before; in the latter case, we have

$$
\begin{aligned}
\int_{D^{\prime}}\left|X_{J}(f)\right|^{2}\left|X_{K}(g)\right|^{2} d V_{0} & \prec\left\|X_{J} f\right\|_{D^{\prime}, 0}^{2} \sup _{x \in D^{\prime}}\left|X_{K} g(x)\right|^{2} \\
& \prec\|f\|_{D,|J|}^{2}\|g\|_{D,|K|+n+2}^{2} \\
& \prec\|f\|_{D, s}^{2}\|g\|_{D, k}^{2},
\end{aligned}
$$

where we have again made use of Lemma 2.1.3. Summing over all terms gives the final estimate.

Lemma 2.2.2. Let $D^{\prime}$ and $D$ be open sets with $D^{\prime} \Subset D \Subset \mathbb{R}^{2 n+1}$, and let $f$ be a function in $\Gamma^{s}(D)$, where $s \geq 2 n+3$. Suppose that $1 / f$ is bounded from above on $\bar{D}^{\prime}$ by a positive constant $C>0$. Then

$$
\|1 / f\|_{D^{\prime}, s} \prec\left(1+\|f\|_{D, s}\right)^{s} .
$$

Consequently, $1 / f$ is contained in $\Gamma^{s}\left(D^{\prime}\right)$. Moreover, if $1 / f^{\prime}<C$ for another function $f^{\prime} \in \Gamma^{s}(D)$ then

$$
\left\|1 / f-1 / f^{\prime}\right\|_{D^{\prime}, s} \prec\left(1+\|f\|_{D, s}\right)^{s}\left(1+\left\|f^{\prime}\right\|_{D, s}\right)^{s}\left\|f-f^{\prime}\right\|_{D, s} .
$$

Proof. We have to estimate the quantities

$$
\int_{D^{\prime}}\left|X_{J}\left(\frac{1}{f}\right)\right|^{2} d V_{0}
$$

for $|J|=t \leq s$. Now, by the quotient and product rules, each such term is bounded by a sum of expressions of the form

$$
\int_{D^{\prime}}\left|\frac{X_{J_{1}} f \cdot X_{J_{2}} f \cdot \ldots X_{J_{p}} f}{f^{p+1}}\right|^{2} d V_{0},
$$

\footnotetext{
${ }^{2}$ We note here that when $|J|=n+1,|J|+n+2=2 n+3$; whence the condition $s \geq 2 n+3$ in the statement of the Lemma.
} 
where $\left|J_{1}\right|+\left|J_{2}\right|+\cdots+\left|J_{p}\right|=t$. Notice that $\left|J_{j}\right|>s / 2$ for at most one multi-index. Hence, computing as in Lemma 2.2.1, we have

$$
\begin{aligned}
\int_{D^{\prime}}\left|\frac{\left(X_{J_{1}} f\right) \cdot\left(X_{J_{2}} f\right) \cdots\left(X_{J_{p}}\right) f}{f^{p+1}}\right|^{2} d V_{0} & \prec C^{2(p+1)} \cdot\left(\|f\|_{D, s}^{2}\right)^{p-1}\|f\|_{D, s}^{2} \\
& \prec\left(1+\|f\|_{D, s}\right)^{s} .
\end{aligned}
$$

Summing over all terms gives the first estimate.

The second estimate follows immediately by applying the first estimate and applying Lemma 2.2 .1 to the quantity $1 / f-1 / f^{\prime}=\left(f^{\prime}-f\right) / f f^{\prime}$.

A minor modification of the proof of Lemma 2.2.1 gives an estimate for the product of several functions.

Lemma 2.2.3. Let $D^{\prime} \Subset D \Subset \mathbb{R}^{2 n+1}$, with $D^{\prime}$ and $D$ open and $s \geq 2 n+4$. Then

$$
\left\|f_{1} \cdots f_{p}\right\|_{D^{\prime}, s} \prec\left\|f_{1}\right\|_{D, s} \cdots\left\|f_{p}\right\|_{D, s}
$$

for all $f_{j} \in \Gamma^{s}(D)$ for $j=1,2, \ldots, p$.

Moreover, for $s>2 n+4$,

$$
\begin{aligned}
& \left\|f_{1} \cdots f_{p}\right\|_{D^{\prime}, s} \\
& \quad \prec \sum_{j=1}^{p}\left\|f_{1}\right\|_{D, s-1} \cdots\left\|f_{j-1}\right\|_{D, s-1}\left\|f_{j}\right\|_{D, s}\left\|f_{j+1}\right\|_{D, s-1} \cdots\left\|f_{p}\right\|_{D, s-1},
\end{aligned}
$$

for all $f_{j} \in \Gamma^{s}(D), j=1,2, \ldots, p$.

Proof. The proof is similar to the proof of Lemma 2.2.1. By the product rule,

$$
\begin{aligned}
\left\|f_{1} \cdots f_{p}\right\|_{D^{\prime}, s}^{2} & \prec \sum_{|J| \leq s} \int_{D^{\prime}}\left|X_{J}\left(f_{1} \cdots f_{p}\right)\right|^{2} d V_{0} \\
& \prec \sum_{\left|J_{1}\right|+\ldots+\left|J_{p}\right| \leq s} \int_{D^{\prime}}\left|X_{J_{1}} f_{1}\right|^{2} \cdots\left|X_{J_{p}} f_{p}\right|^{2} d V_{0},
\end{aligned}
$$

where $X_{I} f$ is defined in Section 2.1. We need only to bound each term in the right-hand summation. Since $s \geq 2 n+4$, it follows that $\left|J_{j}\right|>s / 2 \geq n+2$ for at most one multi-index, say $J_{j}$, in the right-hand sum and that (since $n+2 \leq s / 2)$

$$
\left|J_{i}\right|+n+2 \leq s / 2+n+2 \leq s \text { for } i \neq j
$$

Hence, by Lemma 2.1.3, $X_{J_{i}} f_{i}$ is continuous and $\sup _{x \in D^{\prime}}\left|X_{J_{i}} f_{i}\right| \prec\left\|f_{i}\right\|_{D, s}$. Consequently,

$$
\begin{aligned}
\int_{D^{\prime}}\left|X_{J_{1}} f_{1}\right|^{2} \cdots\left|X_{J_{p}} f_{p}\right|^{2} d V_{0} & \prec \prod_{i \neq j} \sup _{x \in D^{\prime}}\left|X_{J_{i}} f_{i}\right|^{2} \cdot \int_{D^{\prime}}\left|X_{J_{j}} f_{j}\right|^{2} d V_{0} \\
& \prec\left\|f_{1}\right\|_{D, s}^{2} \cdots\left\|f_{p}\right\|_{D, s}^{2}
\end{aligned}
$$

from which the first estimate follows. 
Now suppose that $s>2 n+4$. Then in the previous paragraph $\left|J_{i}\right|+n+2 \leq$ $s-1$; and $\sup _{x \in D^{\prime}}\left|X_{J_{i}} f_{i}\right| \prec\left\|f_{i}\right\|_{D, s-1}$ for $i \neq j$, yielding the estimate

$$
\begin{aligned}
& \left\|f_{1} \cdots f_{p}\right\|_{D^{\prime}, s}^{2} \\
& \prec \sum_{j=1}^{p}\left\|f_{1}\right\|_{D, s-1}^{2} \cdots\left\|f_{j-1}\right\|_{D, s-1}^{2}\left\|f_{j}\right\|_{D, s}^{2}\left\|f_{j+1}\right\|_{D, s-1}^{2} \cdots\left\|f_{p}\right\|_{D, s-1}^{2} \\
& \prec\left(\sum_{j=1}^{p}\left\|f_{1}\right\|_{D, s-1} \cdots\left\|f_{j-1}\right\|_{D, s-1}\left\|f_{j}\right\|_{D, s}\left\|f_{j+1}\right\|_{D, s-1} \cdots\left\|f_{p}\right\|_{D, s-1}\right)^{2} .
\end{aligned}
$$

2.3. The Folland-Stein space of sections of a vector bundle. In this section, we define the Folland-Stein of sections of a vector bundle over a contact manifold.

We begin by extending the definition of the Folland-Stein space $\Gamma^{s}\left(D, \mathbb{R}^{m}\right)$ of functions to the space $\Gamma^{s}\left(M, \mathbb{R}^{m}\right)$ of functions on a compact contact manifold. Let $\left(\phi_{\alpha}, U_{\alpha}, D_{\alpha}\right)$ be an adapted atlas for $M$. A function $f: M \rightarrow \mathbb{R}^{m}$ is said to be a $\Gamma^{s}$-function if the functions $f_{\alpha}=f \circ \phi_{\alpha}^{-1}$ lie in $\Gamma^{s}\left(D_{\alpha}, \mathbb{R}^{m}\right)$ for all $\alpha$. The formula

$$
(f, g)_{M, s}=\sum_{\alpha}\left(f_{\alpha}, g_{\alpha}\right)_{D_{\alpha}, s}
$$

makes $\Gamma^{s}\left(M, \mathbb{R}^{m}\right)$ into a separable Hilbert space. The Sobolev Lemma 2.1.3 clearly extends to this setting:

Lemma 2.3.1. Let $s=k+n+2$, and let $Y_{j}, j=1,2, \ldots, k$ be smooth sections of $H \subset T M$. Then for any function $f \in \Gamma^{s}\left(M, \mathbb{R}^{m}\right)$, the functions $Y_{1} Y_{2} \cdots Y_{k} f$ are continuous on M. Moreover,

$$
\max _{x \in M}\left|Y_{1} Y_{2} \cdots Y_{k} f(x)\right| \prec\|f\|_{M, s}
$$

If $\|f\|_{M, s}<\infty$ for $s=2 k+n+2$, then $f$ is of class $C^{k}$ on $M$. In particular for $s=2 k+n+2$, the linear map

$$
\Gamma^{s}\left(M, \mathbb{R}^{m}\right) \rightarrow C^{k}\left(M, \mathbb{R}^{m}\right)
$$

is continuous.

Similarly, Lemma 2.2.3 assumes the following global form:

Lemma 2.3.2. If $s \geq 2 n+4$

$$
\left\|f_{1} \cdots f_{p}\right\|_{M, s} \prec\left\|f_{1}\right\|_{M, s} \cdots\left\|f_{p}\right\|_{M, s}
$$


and if $s>2 n+4$,

$$
\begin{aligned}
\left\|f_{1} \cdots f_{p}\right\|_{M, s} \prec & \sum_{j=1}^{k}\left\|f_{1}\right\|_{M, s-1} \cdots\left\|f_{j-1}\right\|_{M, s-1}\left\|f_{j}\right\|_{M, s} \\
& \times\left\|f_{j+1}\right\|_{M, s-1} \cdots\left\|f_{p}\right\|_{M, s-1},
\end{aligned}
$$

for all $f_{j} \in \Gamma^{s}(M), j=1,2, \ldots, p$.

We define the Hilbert space of Folland-Stein sections $\Gamma^{s}(E)$ for $\pi: E \rightarrow$ $M$ a smooth vector bundle of rank $m$ as follows. View $\Gamma^{s}\left(M, \mathbb{R}^{r}\right), r \geq 1$, as the Folland-Stein space of sections of the trivial vector bundle $M \times \mathbb{R}^{r} \rightarrow M$. For $r$ sufficiently large, there is a vector bundle injection $E \stackrel{\iota}{\hookrightarrow} M \times \mathbb{R}^{r}$. Define an inner product on $C^{\infty}(E)$ by the formula

$$
(f, g)_{E, s}=(f \circ \iota, g \circ \iota)_{M, s}
$$

for $f, g \in C^{\infty}(E)$, and let $\Gamma^{s}(E)$ be the Hilbert space completion of $C^{\infty}(E)$ with respect to this inner product. It is not difficult to check that, although the inner product depends on $\iota$, the space $\Gamma^{s}(E)$ does not.

Remark 2.3.3. Because $\Gamma^{s}(E)$ is a closed subset of $\Gamma^{s}\left(M, \mathbb{R}^{r}\right)$, the Sobolev Lemma (2.3.1) extends to this setting,

The next proposition is the analogue of "Axiom B2" of Palais (see [Pal, p. 10]) in the setting of contact manifolds.

Proposition 2.3.4. Let $F: M \rightarrow N$ be a smooth contact diffeomorphism between two compact, contact manifolds. Let $E \rightarrow N$ be a smooth vector bundle over $N$ and let $F^{*} E \rightarrow M$ be its pull-back to $M$. Then the map $\sigma \mapsto \sigma \circ F$ is a Hilbert space isomorphism between $\Gamma^{s}(E)$ and $\Gamma^{s}\left(F^{*} E\right)$.

Proof. Choose a bundle injection $\iota: E \hookrightarrow N \times \mathbb{R}^{r}$, and let $\left\{\phi_{\alpha}: U_{\alpha} \rightarrow \mathbb{R}^{2 n+1}\right\}$ be an adapted atlas for $N$ (see Introduction section). Set $U_{\alpha}^{\prime}=F^{-1}\left(U_{\alpha}\right)$ and $\phi_{\alpha}^{\prime}=\phi_{\alpha} \circ F$. Then $\left\{\phi_{\alpha}^{\prime}: U_{\alpha}^{\prime} \rightarrow \mathbb{R}^{2 n+1}\right\}$ is an adapted atlas for $M$. Use this atlas to define the inner product on $\Gamma^{s}\left(M, \mathbb{R}^{r}\right)$. Then by construction

$$
(f \circ F, g \circ F)_{s, M}=(f, g)_{s, N}
$$

for all $f, g \in \Gamma^{s}\left(N, \mathbb{R}^{r}\right)$. Restricting $f$ and $g$ to sections of $E$ then gives the result.

The next proposition shows that $\Gamma^{s}$ satisfies "Axiom B5" of Palais ( [Pal, p. 39] for all $s \geq 2 n+4$.

Proposition 2.3.5. Let $E_{j} \rightarrow M, j=1,2$ be smooth vector bundles over $M$ and let $F: E_{1} \rightarrow E_{2}$ be a smooth (not necessarily linear) fibre-preserving map. Then the map

$$
\Gamma_{F}: \Gamma^{s}\left(E_{1}\right) \rightarrow \Gamma^{s}\left(E_{2}\right): \sigma \mapsto F \circ \sigma
$$

is a $C^{\infty}$ map for all $s \geq 2 n+4$. 
Moreover, if $E_{1}$ is equipped with norm $|\cdot|_{E_{1}}$, then for every $\sigma \in \Gamma^{s}\left(E_{1}\right)$ and every $c>0$ there is a polynomial $Q$ with non-negative coefficients of total degree at most s such that

$$
\left\|F \circ \sigma-F \circ \sigma^{\prime}\right\|_{s} \leq\left\|\sigma-\sigma^{\prime}\right\|_{s} Q^{s}\left(\|\sigma\|_{s},\left\|\sigma^{\prime}\right\|_{s}\right)
$$

for all $\sigma^{\prime} \in \Gamma^{s}\left(E_{1}\right)$, with $\left|\sigma^{\prime}-\sigma\right|<c$.

Proof. Our proof follows a similar argument in [Pal, Theorem 11.3]. We first show that $\Gamma_{F}$ satisfies the polynomial estimate $(*)$, from which continuity of $\Gamma_{F}$ follows. It suffices to work in local coordinates of an adapted atlas for $M$. Then $\Gamma^{s}$ sections of $E_{j}$ can be identified with elements of $\Gamma^{s}\left(D, \mathbb{R}^{m_{j}}\right)$, where $m_{j}$ is the fibre dimension of $E_{j}$. Let $f: D \rightarrow \mathbb{R}^{m_{1}}$ be the local coordinate representation of $\sigma$, and choose a constant $c>0$. Choose $\sigma^{\prime}$ so that its local representative $g \in \Gamma^{S}\left(D, \mathbb{R}^{m_{1}}\right)$ satisfies $\sup _{x \in D}|g(x)-f(x)|<c$. Then in local coordinates $F \circ \sigma(x)=F(x, f(x))$ and $F \circ \sigma^{\prime}(x)=F(x, g(x))$. By smoothness of $F$ and compactness of $\overline{D^{\prime}}$, all derivatives of $F$ are bounded and smooth on the set $\left\{(x, y): x \in \overline{D^{\prime}},|y-f(x)|<c\right.$. Hence, there is a fixed constant $C>0$ such that

$$
\left|F^{(k)}(x, y)\right|<C \quad \text { and } \quad\left|F^{(k)}(x, y)-F^{(k)}(x, z)\right|<C|y-z|
$$

for all $(x, y)$ and $(x, z)$ with $|y-f(x)| \leq c$ and $|z-g(x)| \leq c$, where $F^{(k)}(x, y)$ denotes any mixed partial derivative of $F$ of order $k \leq s$.

Next recall that $\|F \circ f-F \circ g\|_{D^{\prime}, s}^{2}$ is a sum of integrals of the form

$$
\int_{D^{\prime}}\left|X_{I}\{F(x, f(x))-F(x, g(x))\}\right|^{2} d V
$$

for $|I| \leq s$. By the chain rule, $X_{I}\{F(x, f(x))-F(x, g(x))\}$ is a finite sum of terms of the form

(iii)

$$
\begin{aligned}
F^{(k)} & (x, f(x)) \cdot X_{I_{1}} f(x) \cdots X_{I_{k}} f(x)-F^{(k)}(x, g(x)) \cdot X_{I_{1}} g(x) \cdots X_{I_{k}} g(x) \\
= & \left\{F^{(k)}(x, f(x))-F^{(k)}(x, g(x))\right\} \cdot X_{I_{1}} f(x) \cdots X_{I_{k}} f(x) \\
& +\left(F^{(k)}(x, g(x)) \cdot\left\{X_{I_{1}} f(x) \cdots X_{I_{k}} f(x)-X_{I_{1}} g(x) \cdots X_{I_{k}} g(x)\right\},\right.
\end{aligned}
$$

where $0 \leq k \leq s$ and $\sum_{j=1}^{k}\left|I_{j}\right| \leq s$. Applying (i) to (iii) gives the estimate

$$
\begin{aligned}
& \left|F^{(k)}(x, f(x)) \cdot X_{I_{1}} f(x) \cdots X_{I_{k}} f(x)-F^{(k)}(x, g(x)) \cdot X_{I_{1}} g(x) \cdots X_{I_{k}} g(x)\right| \\
& \quad \leq C|f(x)-g(x)| \cdot\left|X_{I_{1}} f(x) \cdots X_{I_{k}} f(x)\right|+C \mid X_{I_{1}} f(x) \cdots X_{I_{k}} f(x) \\
& \quad-X_{I_{1}} g(x) \cdots X_{I_{k}} g(x) \mid
\end{aligned}
$$


The right-hand side of (iv) can, in turn, be bounded by a finite sum of terms form

$$
\begin{aligned}
& C\left|X_{I_{0}}(f(x)-g(x))\right| \cdot\left|X_{I_{1}} f(x)\right| \ldots\left|X_{I_{k^{\prime}}} f(x)\right| \\
& \quad \cdot\left|X_{I_{k^{\prime}+1}} g(x)\right| \ldots\left|X_{I_{k^{\prime}+k^{\prime \prime}}} g(x)\right|
\end{aligned}
$$

where $0 \leq\left|I_{i}\right|$ and $\sum_{i=0}^{k^{\prime}+k^{\prime \prime}}\left|I_{i}\right| \leq s$. Substituting (v) into (ii) shows that $\|F \circ f-F \circ g\|_{D^{\prime}, s}^{2}$ is bounded by a sum of integrals of the form

$$
\begin{aligned}
& \int_{D} C^{2}\left|X_{I_{0}}(f(x)-g(x))\right| \cdot\left|X_{I_{1}} f(x)\right| \cdots\left|X_{I_{k^{\prime}}} f(x)\right| \\
& \quad \cdot\left|X_{I_{k^{\prime}+1}} g(x)\right| \cdots\left|X_{I_{k^{\prime}+k^{\prime \prime}}} g(x)\right|\left|X_{J_{0}}(f(x)-g(x))\right| \cdot\left|X_{J_{1}} f(x)\right| \cdots\left|X_{J_{\ell^{\prime}}} f(x)\right| \\
& \quad \cdot\left|X_{J_{\ell^{\prime}+1}} g(x)\right| \cdots\left|X_{U_{\ell^{\prime}+\ell^{\prime \prime}}} g(x)\right| d V_{0},
\end{aligned}
$$

where $0 \leq\left|J_{j}\right|$ and $\sum_{j=0}^{\ell^{\prime}+\ell^{\prime \prime}}\left|J_{j}\right| \leq s$.

Notice that $\left|I_{i}\right| \geq s / 2$ and $\left|J_{j}\right| \geq s / 2$ for at most one $i$ and at most one $j$, and since $s \geq 2 n+4$, the Sobolev Lemma 2.1.3 applies to show that the remaining factors in the integrand are all continuous, hence bounded on the compact set $\left\{(x, y): x \in \overline{D^{\prime}},|y-f(x)| \leq c\right\}$. It follows that the integral in (vi) is bounded by an expression of the form

$$
C^{\prime}\|f-g\|_{D, s}^{2}\|f\|_{D, s}^{k^{\prime}}\|g\|_{D, s}^{k^{\prime \prime}}\|f\|_{D, s}^{\ell^{\prime}}\|g\|_{D, s}^{\ell^{\prime \prime}}
$$

for $C^{\prime}$ a constant depending on $s, F, c$, and $D^{\prime}$. Since $k^{\prime}+k^{\prime \prime} \leq s$ and $\ell^{\prime}+\ell^{\prime \prime} \leq s$, it follows that

$$
\|F \circ f-F \circ g\|_{D^{\prime}, s} \prec\|f-g\|_{D, s} Q\left(\|f\|_{D, s},\|g\|_{D, s}\right),
$$

where $Q(u, v)$ is a polynomial of bidegree at most $s$ in $u$ and $v$, with nonnegative coefficients. Applying this to each chart in an adapted atlas yields the global estimate $(*)$.

We now show that $\Gamma_{F}$ is $C^{1}$ with derivative given by the formula

$$
d \Gamma_{F}=\Gamma_{\delta F}: \Gamma^{s}\left(E_{1}\right) \times \Gamma^{s}\left(E_{1}\right) \rightarrow \Gamma^{s}\left(E_{2}\right),
$$

where $\delta F: E_{1} \times_{M} E_{1} \rightarrow E_{2}$ is the smooth fibre bundle map defined by the formula

$$
\delta F_{x}(u, v)=\left.\frac{d}{d h}\right|_{h=0} F(u+h v), \text { for all } x \in M \text { and } u, v \in E_{1, x} .
$$

To show that

$$
\lim _{v \rightarrow 0} \frac{\left\|\Gamma_{F}(\sigma+v)-\Gamma_{F}(\sigma)-\Gamma_{\delta F}(\sigma, v)\right\|_{s}}{\|v\|_{s}}=0,
$$

first observe that $\delta F$ can be expressed as a smooth map of the form

$$
\delta F: E_{1} \rightarrow \operatorname{Hom}\left(E_{1}, E_{2}\right)
$$


Hence,

$$
\Gamma(\delta F): \Gamma^{s}\left(E_{1}\right) \rightarrow \Gamma^{s}\left(\operatorname{Hom}\left(E_{1}, E_{2}\right)\right)
$$

is continuous, and for all $\epsilon>0$ there is a $\delta_{1}$ such that

$$
\|\Gamma(\delta F)(\sigma+v)-\Gamma(\delta F)(\sigma)\|_{s}<\epsilon, \text { whenever }\|v\|_{s}<\delta_{1} .
$$

Using this observation, we compute as follows:

$$
\begin{aligned}
\Gamma_{F}(\sigma+v)-\Gamma_{F}(\sigma)-\Gamma_{\delta F}(\sigma, v) & =F(\sigma+v)-F(\sigma)-\delta F_{\sigma} \cdot v \\
& =\int_{0}^{1}\left\{\delta F_{\sigma+t v} \cdot v-\delta F_{\sigma} \cdot v\right\} d t \\
& =\int_{0}^{1}\left\{\Gamma_{\delta F}(\sigma+t v)(v)-\Gamma_{\delta F}(\sigma)(v)\right\} d t .
\end{aligned}
$$

Hence, if $\|v\|_{s}<\delta_{1}$ then

$$
\begin{aligned}
& \left\|\Gamma_{F}(\sigma+v)-\Gamma_{F}(\sigma)-\Gamma_{\delta F}(\sigma, v)\right\|_{s} \\
& \quad \leq \int_{0}^{1}\left\|\Gamma_{\delta F}(\sigma+t v)(v)-\Gamma_{\delta F}(\sigma)(v)\right\|_{s} d t<\epsilon\|v\|_{s} .
\end{aligned}
$$

This show that $\Gamma_{F}$ is differentiable at $\sigma$. That it is continuously differentiable follows from the identity $d \Gamma_{F}=\Gamma_{\delta F}$ and continuity of $\Gamma_{\delta F}$.

That $\Gamma_{F}$ is smooth follows by induction. For assume that for some $k>0$, $\Gamma_{F}$ is $C^{k}$, for all smooth $F: E_{1} \rightarrow E_{2}$, and all $E_{j}$. To show that $\Gamma_{F}$ is $C^{k+1}$, we need only to show that its derivative $d \Gamma_{F}: \Gamma^{s}\left(E_{1} \times E_{1}\right) \rightarrow \Gamma^{s}\left(E_{2}\right)$ is $C^{k}$. But $d \Gamma_{F}=\Gamma_{\delta F}$ and $\delta F$ is a smooth fibre bundle map. Consequently, $d \Gamma_{F}$ is $C^{k}$, completing the induction step.

2.4. The Folland-Stein space of sections of a fibre bundle. In this section, we define the Folland-Stein space $\Gamma^{s}(E)$ of sections of $E$ for $s \geq$ $2 n+4$ in the case where $\pi: E \rightarrow M$ is a smooth fibre bundle over $M$. For this range of $s, \Gamma^{s}(E)$ is a smooth infinite-dimensional manifold modelled on the Folland-Stein space of sections of certain vector bundles. The construction and the proof are due to Palais (see [Pal, Chapters 12 and 13] for details). We emphasize that this construction depends heavily on Propositions 2.3.4 and 2.3.5 (Palais' Axioms B2 and B5), which are satisfied for $s \geq 2 n+4$.

Let $\pi: E \rightarrow M$ be a fibre bundle, and choose a smooth section $\sigma \in$ $C^{\infty}(E)$. The bundle of vertical tangent vectors along $\sigma$ is the vector bundle defined by

$$
T_{\sigma}(E)=\left\{X \in T E_{\sigma(x)}: x \in M, d \pi(X)=0\right\} .
$$

Palais shows that there is a smooth fibre bundle isomorphism

$$
\psi_{\sigma}: T_{\sigma}(E) \stackrel{\simeq}{\longrightarrow} \mathcal{O}_{\sigma} \stackrel{\text { open }}{\subset} E,
$$


where $\mathcal{O}_{\sigma}$ is a neighbourhood of the image of $\sigma$. Palais also shows that the the image of every continuous section of $E$ is contained in a set of the form $\mathcal{O}_{\sigma}$ for some smooth section $\sigma$.

Consequently, every continuous section of $E$ can be identified with a continuous section of $T_{\sigma}(E)$ for some $\sigma \in C^{\infty}(E)$, and $C^{0}(E)$ can we written as the following union of open sets:

$$
C^{0}(E)=\bigcup_{\sigma \in C^{\infty}(E)} C^{0}\left(T_{\sigma}(E)\right)
$$

Since $s \geq 2 n+4 \geq n+2$, Lemma 2.3.1 applies to give continuous inclusions $\Gamma^{s}\left(T_{\sigma}(E)\right) \subset C^{0}\left(T_{\sigma}(E)\right)$.

We may thus define the Folland-Stein space $\Gamma^{s}(E)$ to be the union

$$
\Gamma^{s}(E)=\bigcup_{\sigma \in C^{\infty}(E)} \Gamma^{s}\left(T_{\sigma}(E)\right),
$$

equipped with the weakest topology such that $\Gamma_{\psi_{\sigma}}: \Gamma^{s}\left(T_{\sigma}(E)\right) \rightarrow \Gamma^{s}(E)$ is a continuous open map for all $\sigma \in C^{\infty}(E)$.

In fact, $\Gamma^{s}(E)$ is a Hilbert manifold with $C^{\infty}$ atlas given by the charts $\Gamma\left(\psi_{\sigma}^{-1}\right)$; and as Palais shows, smoothness of the transition functions for this atlas follows from Proposition 2.3.5.

The above construction is functorial:

Proposition 2.4.2 (Palais, Theorem 13.4). Let $E_{j} \rightarrow M, j=1,2$ be smooth fibre bundles over $M$ and let $F: E_{1} \rightarrow E_{2}$ be a smooth fibrepreserving map. Then the map

$$
\Gamma(F): \Gamma^{s}\left(E_{1}\right) \rightarrow \Gamma^{s}\left(E_{2}\right): \sigma \mapsto F \circ \sigma
$$

is a $C^{\infty}$ map of Hilbert manifolds for all $s \geq 2 n+4$.

Remark 2.4.3. By construction, the Sobolev Lemma 2.3.1 extends to define a continuous injection $\Gamma^{s}(E) \subset C^{k}(E)$, for $s=\max (2 k+n+2,2 n+4)$.

The case where $E$ is the trivial fibre bundle $E=M \times N \rightarrow M$ is an important special case:

Definition 2.4.4. Let $N$ be a smooth manifold without boundary. For $s \geq 2 n+4$, the Folland-Stein space $\Gamma^{s}(M, N)$ of maps from the contact manifold $M$ to the manifold $N$ is the Folland-Stein space of sections of the trivial fibre bundle $M \times N \rightarrow M$.

Corollary 2.4.5. Let $F: N \rightarrow N^{\prime}$ be a $C^{\infty}$ map between $C^{\infty}$ manifolds. Left composition with $F$ defines a $C^{\infty}$

$$
L_{F}^{s}: \Gamma^{s}(M, N) \rightarrow \Gamma^{s}\left(M, N^{\prime}\right): G \mapsto F \circ G
$$

for all $s \geq 2 n+4$. If $F: N \rightarrow N$ is a diffeomorphism of $N$ then $L_{F}^{s}$ is a diffeomorphism of $\Gamma^{s}(M, N)$. 
Proof. View composition with $F$ as a smooth bundle map $(x, y) \mapsto(x, F(y))$, and apply Proposition 2.4.2. If $F$ is a diffeomorphism then $R_{F^{-1}}^{s}$ is the smooth inverse of $R_{F}^{s}$.

2.5. Horizontal jets and differential operators. The goal of this section is to extend the framework of [Pal, Chapter 15] to the context of differential operators on contact manifolds.

Let $\pi: E \rightarrow M$ be a smooth fibre bundle over $M$, and choose an adapted coordinate chart

$$
\psi: U \rightarrow \phi(V) \times \mathbb{R}^{m}: p \mapsto(x, y),
$$

as defined in Section 1.1. Two smooth local sections $\sigma_{i}, i=1,2$, of $E$ defined on $V$, with local representations $f_{i}: \phi(V) \rightarrow \mathbb{R}^{m}$, are said to be contact equivalent up to order $k$ at a point $p \in V$ if and only if

$$
X_{I} f_{1}(\phi(p))=X_{I} f_{2}(\phi(p))
$$

for every multi-index $I$ with $0 \leq|I| \leq k$. It is easy to check that contact equivalence is an equivalence relation and that it is independent of coordinates. The horizontal $k$-jet of $\sigma$ at $p$, written $j_{H}^{k} \sigma(p)$, is the equivalence class of the local section $\sigma$ at $p \in M, J_{H}^{k} E$ denotes the space of all horizontal $k$-jets. The map $\pi: J_{H}^{k} E \rightarrow M$ defined by

$$
\pi\left(j_{H}^{k} \sigma(p)\right)=p
$$

makes the space of horizontal $k$-jets into a fibre bundle with fibres of dimension $m \cdot N_{k}$, where $N_{k}$ is the number of indices $A$ with $|A| \leq k$.

Remark 2.5.2. By virtue of the commutation relations among the vector fields $T_{0}, X_{1}, \ldots X_{2 n}$, any differential operator of the form $Y_{1} Y_{2} \cdots Y_{r}$, where $Y_{1}, \ldots, Y_{r}$ are arbitrary vector fields on an open set $V \subset M$, can be expressed uniquely in local coordinates as a linear combination of operators of the form

$$
D_{A}:=\underbrace{X_{1} \cdots X_{1}}_{a_{1}}, \underbrace{X_{2} \cdots X_{2}}_{a_{2}}, \ldots, \underbrace{X_{2 n} \cdots X_{2 n}}_{a_{2 n}} \underbrace{T_{0} \cdots T_{0}}_{a_{2 n+1}},
$$

where $A=\left(a_{1}, a_{2}, \ldots, a_{2 n}, a_{2 n+1}\right), 0 \leq a_{j}$. Moreover, if $Y_{1}, \ldots, Y_{r}$ are all horizontal, then $a_{1}+a_{2}+\cdots+a_{2 n}+2 a_{2 n+1}=r$. The integer $|A|=a_{1}+$ $\cdots+a_{2 n}+2 a_{2 n+1}$ is called the contact order of $D_{A}$. It follows that $\sigma_{1}$ and $\sigma_{2}$ are contact equivalent up to order $k$ at $p$ if and only if

$$
D_{A} f_{1}(\phi(p))=D_{A} f_{2}(\phi(p))
$$

for all multi-indices $A$ with $|A| \leq k$.

Lemma 2.5.3. Let $E \rightarrow M$ be a smooth fibre bundle with fibre dimension $m$. Then $J_{H}^{k} E \rightarrow M$ is a smooth fibre bundle. Moreover, if $\sigma$ is a smooth section 
of $E$ then $j_{H}^{k} \sigma: p \mapsto j_{H}^{k} \sigma(p)$ is a smooth section of $J_{H}^{k} E$. If $F: E_{1} \rightarrow E_{2}$ is a smooth map of fibre bundles, then so is the map

$$
J_{H}^{k}(F): J_{H}^{k} E_{1} \rightarrow J_{H}^{k} E_{2}: j_{H}^{k} \sigma(p) \mapsto j_{H}^{k}(F \circ \sigma)(p) .
$$

This construction is functorial, i.e., $J_{H}^{k}(G \circ F)=J_{H}^{k}(G) \circ J_{H}^{k}(F)$, for $G$ : $E_{2} \rightarrow E_{3}$ a smooth map of fibre bundles.

Proof. We give an outline of the proof, leaving some details to the reader. To define a coordinate chart for $J_{H}^{k} E$, choose a point $q_{0} \in E$ and let $p_{0}=$ $\pi\left(q_{0}\right)$. Let

$$
\psi: U \rightarrow \phi(V) \times \mathbb{R}^{n}: q \mapsto(x, y)
$$

be adapted coordinates for $E$ centred at $q_{0}$ (see Section 1.1); and let $\widetilde{U}$ be the set of horizontal $k$-jets of sections of $E$ defined on $V$ and with values in $U \subset E$. Viewing $\mathbb{R}^{m \cdot N_{k}}$ as the space of $m \times N_{k}$ matrices, we can define local fibre bundle coordinates

$$
\widetilde{\psi}: \widetilde{U} \rightarrow \mathbb{R}^{2 n+1} \times \mathbb{R}^{m \cdot N_{k}}
$$

by the formula

$$
\widetilde{\psi}\left(j_{H}^{k} \sigma(p)\right)=\left((x(p)),\left(D_{A} f_{\sigma}(p)\right)\right),
$$

where $f_{\sigma}$ is the local representation of $\sigma$ (Section 1.1), $D_{A} f_{\sigma}$ is the derivative of $f_{\sigma}$ with respect to the multi-index $A$, and we have given the set $\{A:|A| \leq k\}$ the lexicographical ordering. We need only to show that $\widetilde{\psi}$ is a bijection between $\widetilde{U}$ and $\phi(V) \times \mathbb{R}^{m \cdot N_{k}}$ for $U$ a sufficiently small neighbourhood of $q_{0}$. By the discussion in Remark 2.5.2, $\widetilde{\psi}$ is injective.

To prove surjectivity, choose a point $S=\left\{S_{A} \in \mathbb{R}^{m}:|A| \leq k\right\} \in \mathbb{R}^{m \cdot N_{k}}$, and consider the polynomial section

$$
y=\sigma_{S}(x)=\sum_{A} \frac{1}{A !} S_{A} \cdot\left(x^{1}\right)^{a_{1}} \cdots\left(x^{2 n}\right)^{a_{2 n}}\left(x^{2 n+1}\right)^{a_{2 n+1}},
$$

where we have adopted the notation $A !=a_{1} ! \cdots a_{2 n} ! a_{2 n+1} !$. Now for any point $p=\left(x^{1}, \ldots, x^{2 n+1}\right)$, the map

$$
S \mapsto \widetilde{\psi}\left(\sigma_{S}(p)\right)=\left(x, D_{A} \sigma_{S}(x)\right)
$$

may be viewed as a linear map $L_{x}: \mathbb{R}^{m \cdot N_{k}} \rightarrow \mathbb{R}^{m \cdot N_{k}}$. Note that $L_{x}$ depends smoothly on $x$. To show that $L_{x}$ is a bijection for $x$ sufficiently near 0 , we need only to verify that $L_{0}$ is injective. But a straightforward computation shows that

$$
D_{A^{\prime}} \sigma_{S}(0)= \begin{cases}S_{A} & \text { for } A^{\prime}=A \\ 0 & \text { otherwise }\end{cases}
$$


i.e., $L_{0}$ is the identity map. This shows that $\widetilde{\psi}$ is surjective on the fibre of $J_{H}^{k} E$ over all points $p$ sufficiently near $p_{0}$. Thus, after a possible shrinking of $U$, the map

$$
\widetilde{\psi}: \widetilde{U} \rightarrow \mathbb{R}^{2 n+1} \times \mathbb{R}^{m \cdot N_{k}}
$$

is a bijection between $\widetilde{U}$ and $\phi(U) \times \mathbb{R}^{m \cdot N_{k}}$.

By letting $q_{0}$ vary over all $E$, we obtain a smooth atlas for $J_{H}^{k} E$. We topologize $J_{H}^{k} E$ by requiring each of the charts $\widetilde{\psi}$ to be a homeomorphism. That these charts form a smooth atlas making $J_{H}^{k} E$ into a smooth manifold follows from the observation that if $\widetilde{\psi}$ and $\widetilde{\psi}^{\prime}$ are two charts then $\widetilde{\psi}^{\prime} \circ \widetilde{\psi}^{-1}$ is a diffeomorphism between $\widetilde{\phi}\left(\widetilde{U} \cap \widetilde{U}^{\prime}\right)$ and $\widetilde{\phi^{\prime}}\left(\widetilde{U} \cap \widetilde{U}^{\prime}\right)$. The proof follows by standard arguments in advanced calculus (i.e., the Inverse Function theorem and the Chain rule) and is left to the reader.

That $J_{H}^{k}(F)$ and $j_{H}^{k} \sigma$ are smooth follows from the Chain Rule, as does the identity $J_{H}^{k}(G \circ F)=J_{H}^{k}(G) \circ J_{H}^{k}(F)$.

Remark 2.5.4. In the special case where $E \rightarrow M$ is a smooth vector bundle, then so is $J_{H}^{k} E \rightarrow M$, with linear structure induced by the formula

$$
a_{1} j_{H}^{k} \sigma_{1}(p)+a_{2} j_{H}^{k} \sigma_{2}(p)=j_{H}^{k}\left(a_{1} \sigma_{1}+a_{2} \sigma_{2}\right)(p) .
$$

Lemma 2.5.5. Let $E \rightarrow M$ be a smooth fibre bundle over $M$. Then the map

$$
\Gamma\left(j_{H}\right): \Gamma^{s}(E) \rightarrow \Gamma^{s-k}\left(J_{H}^{k} E\right): \sigma \mapsto j_{H}^{k} \sigma
$$

is a smooth map of Hilbert manifolds for all $k$ and $s$ such that $s \geq 2 n+4+k$. In the special case where $E$ is a vector bundle, $\Gamma\left(j_{H}\right)$ is a bounded linear map of Hilbert spaces.

Proof. From the discussion in Section 2.1, it suffices to analyse $\Gamma\left(j_{H}\right)$ in the neighbourhood of a fixed section of $E$. By definition of the Hilbert manifold structure of $\Gamma^{s}(E)$, we may, without loss of generality, assume that $E$ is a vector bundle, and we must only show that the linear map $\Gamma\left(j_{H}\right)$ is bounded. By definition of the inner product on $\Gamma^{s}(E)$, the result then follows from a local computation: choose an open set $V \Subset M$ and a trivialization $E_{\mid \bar{V}} \simeq$ $\bar{V} \times \mathbb{R}^{m}$. Then a section $\sigma$ of $E$ over $V$ is given by an $\mathbb{R}^{m}$-valued function $f_{\sigma}$, and its horizontal $k$-jet $j_{H}^{k} \sigma: V \rightarrow J_{H}^{k} M$ can be identified with the $m \times N_{k}$ matrix-valued function

$$
j_{H}^{k} \sigma=\left(D_{A} f_{\sigma}\right)_{|A| \leq N_{k}}
$$


So

$$
\begin{aligned}
\left\|j_{H}^{k} \sigma\right\|_{V, s-k}^{2} & =\sum_{j} \sum_{0 \leq|J| \leq s-k} \int_{V}\left|X_{J}\left(j_{H}^{k} \sigma^{j}\right)\right|^{2} d V_{0} \\
& =\sum_{0 \leq|J| \leq s-k} \sum_{|A| \leq k} \int\left|X_{J} D_{A} f_{\sigma}\right|^{2} d V_{0} \\
& \leq \sum_{0 \leq|I| \leq s} \int\left|X_{I} f_{\sigma}\right|^{2} d V_{0} \leq\|\sigma\|_{V, s}^{2} .
\end{aligned}
$$

Definition 2.5.6. Let $E_{1}$ and $E_{2}$ be smooth fibre bundles over $M$. A differential operator of contact order $k$ from $E_{1}$ to $E_{2}$ is a map of the form

$$
D: C^{\infty}\left(E_{1}\right) \stackrel{j_{H}^{k}}{\longrightarrow} C^{\infty}\left(J_{H}^{k} E_{1}\right) \stackrel{F_{*}}{\longrightarrow} C^{\infty}\left(E_{2}\right),
$$

where $F: J_{H}^{k} E_{1} \rightarrow E_{2}$ is a smooth fibre bundle map and $F_{*}$ is defined by the formula

$$
F_{*}(\hat{\sigma})=F \circ \hat{\sigma} \text { for } \hat{\sigma} \in C^{\infty}\left(J_{H}^{k} E_{1}\right) .
$$

Proposition 2.5.7. Let $D$ be a differential operator of contact order $k$ as above. Then $D$ extends to a smooth map

$$
D: \Gamma^{s}\left(E_{1}\right) \stackrel{j_{H}^{k}}{\longrightarrow} \Gamma^{s-k}\left(J_{H}^{k} E_{1}\right) \stackrel{F_{*}}{\longrightarrow} \Gamma^{s-k}\left(E_{2}\right)
$$

for all $s \geq 2 n+4+k$.

In the special case where $E_{1}$ and $E_{2}$ are normed vector bundles, for every section $\sigma \in \Gamma^{s}\left(E_{1}\right)$ and every constant $c>0$, there is a polynomial $Q$ of degree at most $s-k$ such that the estimate

$$
\left\|D \sigma-D \sigma^{\prime}\right\|_{s-k} \leq\left\|\sigma-\sigma^{\prime}\right\|_{s} \cdot Q\left(\|\sigma\|_{s},\left\|\sigma^{\prime}\right\|_{s}\right)
$$

holds for every section $\sigma^{\prime} \in \Gamma^{s}\left(E_{1}\right)$ satisfying $\left|j_{H}^{k} \sigma^{\prime}\right| \leq c$.

Proof. The first part of the proposition is an immediate corollary to Propositions 2.5.5 and 2.3.5. The second part of the proposition follows from estimate in Proposition 2.3.5.

Remark 2.5.8. The restriction $s \geq 2 n+4+k$ in Proposition 2.5.7 can be relaxed to $s \geq k$ when $D$ is a linear operator, and the estimate assumes the form $\|D \sigma\|_{s-k} \leq C\|\sigma\|_{s}$. When $D$ is non-linear, the term $\|D \sigma\|_{s-k}$ involves products of $\sigma$ and its derivatives, and estimating these expressions uses Lemma 2.2.3, which assumes $s-k \geq 2 n+4$.

Examples 2.5.9. As examples, we consider the contact order of some basic operators that we need later. Let $M$ be a contact manifold with contact form $\eta$ and characteristic vector field $T$. 
(i) Lie differentiation with respect to the Reeb vector field $\mathcal{L}_{T}: \Gamma^{s}(M) \rightarrow$ $\Gamma^{s-2}(M): f \mapsto T(f)$ is a differential operator of contact order 2 . To see this, we work locally, using an adapted coordinate chart $\phi: U \rightarrow$ $\mathbb{R}^{2 n+1}$ as in Section 1.1. Note that $\phi_{*} T=T_{0}=\frac{\partial}{\partial x^{2 n+1}}$. But since $T_{0}=\left[X_{1}, X_{n+1}\right]$, the Lie bracket of the two horizontal vector fields, it follows that $T$ has contact order 2 .

(ii) The exterior derivative operator $d: \Gamma^{s}\left(\Lambda^{p} M\right) \rightarrow \Gamma^{s-2}\left(\Lambda^{p+1} M\right)$ : $\alpha \mapsto d \alpha$ is a differential operator of contact order 2 . We again work locally. First consider the case $p=0$, where $\alpha=f$, for $f$ a scalar function on $M$. In this case,

$$
d f=\sum_{k=1}^{2 n} X_{k}(f) d x^{k}+T_{0}(f) \eta_{0} .
$$

Since the term $T_{0}(f) \eta_{0}$ has contact order 2 , and all other terms depend only on the horizontal 1 -jet of $f$, we see that $d \alpha$ has contact order 2 . For $p>0, \alpha$ can be expressed in the form

$$
\alpha=\sum_{I} f_{I} d x^{I}+\sum_{K} g_{K} \eta_{0} \wedge d x^{K},
$$

where $d x^{I}=d x^{i_{1}} \wedge \cdots \wedge d x^{i_{p}}$ and $\eta_{0} \wedge d x^{K}=\eta_{0} \wedge d x^{k_{1}} \wedge \cdots \wedge d x^{k_{p-1}}$, where summation ranges over indices $I$ and $K$ of the forms $i_{1}<i_{2}<$ $\cdots<i_{p}<2 n+1$ and $k_{1}<k_{2}<\cdots<k_{p-1}<2 n+1$. Hence,

$$
d \alpha=\sum_{I} d f_{I} \wedge d x^{I}+\sum_{K} d g_{K} \wedge \eta_{0} \wedge d x^{K}+\sum_{K} g_{K} d \eta_{0} \wedge d x^{K},
$$

which is clearly of contact order 2 .

(iii) Exterior differentiation followed by projection onto $\Lambda^{*} H^{*}$ is a differential operator of contact order 1:

$$
\left.d_{H}: \Gamma^{s}\left(\Lambda^{p} M\right) \rightarrow \Gamma^{s-1}\left(\Lambda^{p+1} H^{*}\right): \alpha \mapsto d \alpha-\eta \wedge(T\lrcorner d \alpha\right) .
$$

To see this, let $\alpha$ be given in local coordinates as in (ii). Then an easy computation gives

$$
d_{H} \alpha=\sum_{I} d_{H} f_{I} \wedge d x^{I}+\sum_{K} g_{K} d \eta_{0} \wedge d x^{K} .
$$

But $d_{H} f_{I}=\sum_{k=1}^{2 n} X_{k}\left(f_{I}\right) d x^{k}$, which is of contact order 1 .

(iv) Now suppose $s \geq 2 n+4$, let $E \rightarrow M$ be a smooth fibre bundle, and let $\beta$ be a fixed, smooth $p$-form on the total space $E$. The restriction on $s$ ensures that the Folland-Stein space $\Gamma^{s}(E)$ is well-defined. (Since $\Lambda^{p} M \rightarrow M$ is a vector bundle, the restriction $s-2 \geq 2 n+4$ is not required to define $\Gamma^{s-2}\left(\Lambda^{p} M\right)$.) We claim that the differential operator

$$
P_{\beta}: \Gamma^{s}(E) \rightarrow \Gamma^{s-2}\left(\Lambda^{p} M\right): \sigma \mapsto \sigma^{*} \beta
$$


is a differential operator of contact order 2. To see this, we work in adapted product coordinates $(x, y) \in \mathbb{R}^{2 n+1} \times \mathbb{R}^{m}$ on $E$ (see Section 1.1). A section $\sigma$ of $E$ is then represented locally by a function $y=f_{\sigma}(x)$, which (by the Sobolev lemma) is at least of class $C^{1}$. The form $\beta$ can be written

$$
\begin{aligned}
\beta= & \sum_{|I|+|K|=p} A_{I, K}(x, y) d x^{I} \wedge d y^{K} \\
& +\sum_{|I|+|K|=p-1} B_{I, K}(x, y) \eta_{0} \wedge d x^{I} \wedge d y^{K},
\end{aligned}
$$

where $I$ and $K$ are the obvious increasing multi-indices. Note that the pullback $\sigma^{*} \beta$ is obtained by setting $y=f_{\sigma}(x)$ and expanding. But for a function $f_{\sigma}$, the differential $f_{\sigma} \mapsto d f_{\sigma}$ is a differential operator of contact order 2 , since it involves derivatives in the $T_{0}$ direction. Applying this observation to each term in the above expansion of $\beta$ shows that $\sigma \mapsto \sigma^{*} \beta$ has contact order 2 .

(v) For $s \geq 2 n+4, E$ and $\beta$ as above,

$$
P_{H, \beta}: \Gamma^{s}(E) \rightarrow \Gamma^{s-1}\left(\Lambda^{p} H^{*}\right): \sigma \mapsto \pi_{H} \sigma^{*} \beta
$$

is a differential operator of contact order 1. Using the expansion (2.5.10) above, we have the local identity

$$
\eta \wedge \sigma^{*} \beta=\sum_{|I|+|K|=p} A_{I, K}(x, y) \eta_{0} \wedge d x^{I} \wedge d f_{\sigma}^{K} .
$$

Now observe that

$$
d f_{\sigma}=\sum_{j=1}^{n} X_{j}\left(f_{\sigma}\right) d x^{j}+X_{n+j}\left(f_{\sigma}\right) d x^{n+j}+T_{0}\left(f_{\sigma}\right) \eta_{0} .
$$

It follows that the local expression for $\eta \wedge \sigma^{*} \beta$ only involves first derivatives of $f_{\sigma}$ with respect to $X_{1}, \ldots, X_{2 n}$. Hence, $\sigma \mapsto \eta \wedge \sigma^{*} \beta$ has contact order 1 . To see that $\sigma \mapsto \pi_{H} \sigma^{*} \beta$ has contact order 1 , note that by equation (1.1.1), $\left.\pi_{H} \sigma^{*} \beta=T\right\lrcorner\left(\eta \wedge \sigma^{*} \beta\right)$. Since $\left.\alpha \mapsto T\right\lrcorner \alpha$ is a smooth map of vector bundles, it preserves $\Gamma^{s}$-spaces. Hence, the composition $\left.\sigma \mapsto \eta \wedge \sigma^{*} \beta \mapsto T\right\lrcorner\left(\eta \wedge \sigma^{*} \beta\right)$ also has contact order 1 .

2.6. Rumin's complex. In $[\mathbf{R}]$, Rumin constructed a novel resolution

$$
\begin{aligned}
& 0 \hookrightarrow \mathbb{R} \longrightarrow \mathcal{R}^{0} \stackrel{d_{R}}{\longrightarrow} \mathcal{R}^{1} \stackrel{d_{R}}{\longrightarrow} \cdots \stackrel{d_{R}}{\longrightarrow} \mathcal{R}^{n} \stackrel{D_{R}}{\longrightarrow} \mathcal{R}^{n+1} \stackrel{d_{R}}{\longrightarrow} \mathcal{R}^{n+2} \\
& \stackrel{d_{R}}{\longrightarrow} \cdots \stackrel{d_{R}}{\longrightarrow} \mathcal{R}^{2 n+1} \longrightarrow 0
\end{aligned}
$$

of the constants on a contact manifold. In this section, we give a brief sketch Rumin's construction. 
For $n<p \leq 2 n+1, R^{p}$ denotes the subbundle of $\Lambda^{p} M$ given by

$$
R^{p}:=\left\{\beta \in \Lambda^{p} M: \eta \wedge \beta=0 \text { and } d \eta \wedge \beta=0\right\}
$$

and for $0 \leq p \leq n, R^{p}$ denotes the quotient bundle $R^{p}=\Lambda^{p}(M) / I^{p}$, where $I^{0}=0$ and

$$
I^{p}:=\left\{\eta \wedge \alpha+d \eta \wedge \beta: \alpha \in \Lambda^{p-1} M, \beta \in \Lambda^{p-2} M\right\} \text { for } 0<p \leq n .
$$

Also note that for $0 \leq p \leq n, R^{p}$ can be written as a quotient bundle of $\Lambda^{p} H^{*}$ :

$$
\tau: \Lambda^{p} H^{*} \rightarrow R^{p}=\Lambda^{p} H^{*} /\left(d \eta \wedge \Lambda^{p-2} H^{*}\right) .
$$

Let $\mathcal{R}^{p}=C^{\infty}\left(R^{p}\right)$. Then $\mathcal{R}^{0}=\Omega^{0}(M)$, and since we make the identification $H^{*}=R^{1}$ with the annihilator of $T$,

$$
\left.\mathcal{R}^{1} \equiv\left\{\alpha \in \Omega^{1}(M): T\right\lrcorner \alpha=0\right\} .
$$

The linear differential operators $d_{R}$ and $D_{R}$ are induced by the exterior derivative operator on forms. Let $\beta \in \mathcal{R}^{p}$ be any section of $R^{p}$. There are three cases to consider:

(i) For $p>n$, set $d_{R} \beta=d \beta$. It is easy to see that $d \beta$ is a section of $R^{p+1}$.

(ii) For $p<n$, set $d_{R} \beta=\pi_{R}(d \widetilde{\beta})$, where $\widetilde{\beta} \in \Omega^{p}(M)$ is any $p$-form with $\pi_{R} \widetilde{\beta}=\beta$ and $\pi_{R}: \Lambda^{p} M \rightarrow R^{p}$ denotes the quotient map. It is not difficult to check that $d_{R} \beta$ is independent of the choice of $\widetilde{\beta}$.

(iii) For $p=n$, set $D_{R}(\beta)=d \widetilde{\beta}$, where $\widetilde{\beta} \in \Omega^{n}(M)$ is an $n$-form satisfying the conditions $\pi_{R} \widetilde{\beta}=\beta$ and $d \widetilde{\beta} \in \mathcal{R}^{n+1}$. Rumin shows that a form $\widetilde{\beta}$ satisfying these conditions exists and that $d \widetilde{\beta}$ is independent of the choice of $\widetilde{\beta}$.

Rumin also shows that $d_{R}$ and $D_{R}$ are linear differential operators, with $d_{R}$ of contact order 1 and $D_{R}$ of contact order 2 . Using the star operator, Rumin proves that $\mathcal{R}^{k}$ is dual to $\mathcal{R}^{2 n+1-k}$ and that the adjoint operators satisfy the identities

$$
\delta_{R}=(-1)^{k} * d_{R} * \text { for } k \neq(n+1) \text { and } D_{R}^{*}=(-1)^{n+1} * D_{R} * .
$$

Thus, $\delta_{R}$ has contact order 1 and $D_{R}^{*}$ has contact order 2 . The next proposition then follows from Proposition 2.5.7 above.

Proposition 2.6.2 (Rumin). Let $\left(M^{2 n+1}, \eta\right)$ be a compact contact manifold with adapted metric $g$ and the associated complex $\left(\mathcal{R}^{*}, d_{R}\right)$. Then the following estimates hold:

$$
\begin{aligned}
& \left\{\begin{array}{ll}
\left\|d_{R} \alpha\right\|_{s-1} \leq c_{s}\|\alpha\|_{s}, & \text { for } k \neq n \\
\left\|D_{R} \alpha\right\|_{s-2} \leq c_{s}\|\alpha\|_{s}, & \text { for } k=n ;
\end{array}\right. \text { and } \\
& \begin{cases}\left\|\delta_{R} \alpha\right\|_{s-1} \leq c_{s}\|\alpha\|_{s}, & \text { for } k \neq n+1 \\
\left\|D_{R}^{*} \alpha\right\|_{s-2} \leq c_{s}\|\alpha\|_{s}, & \text { for } k=n+1 .\end{cases}
\end{aligned}
$$


Rumin $[\mathbf{R}]$ establishes a Hodge theory for this complex. The Laplace operators $\Delta_{R}: \mathcal{R}^{k} \rightarrow \mathcal{R}^{k}$ are defined as follows:

$$
\Delta_{R}= \begin{cases}(n-k) d_{R} \delta_{R}+(n-k+1) \delta_{R} d_{R}, & \text { for } 0 \leq k \leq(n-1), \\ \left(d_{R} \delta_{R}\right)^{2}+D_{R}^{*} D_{R}, & \text { for } k=n, \\ D_{R} D_{R}^{*}+\left(\delta_{R} d_{R}\right)^{2}, & \text { for } k=n+1, \\ (n-k+1) d_{R} \delta_{R}+(n-k) \delta_{R} d_{R}, & \text { for }(n+2) \leq k \leq(2 n+1) .\end{cases}
$$

Theorem 2.6.4 (Rumin). Let $M$ be a contact manifold with adapted metric $g$. The Laplace operators $\Delta_{R}$ are maximal hypoelliptic, and the following estimates are satisfied for $\alpha \in \mathcal{R}^{k}$ :

$$
\begin{cases}\|\alpha\|_{s+2} \leq c_{s}\left\|\Delta_{R} \alpha\right\|_{s}+\|\alpha\|_{0}, & \text { for } k \neq n,(n+1), \\ \|\alpha\|_{s+4} \leq c_{s}\left\|\Delta_{R} \alpha\right\|_{s}+\|\alpha\|_{0}, & \text { for } k=n,(n+1) .\end{cases}
$$

Remark 2.6.5. Rumin only establishes the estimates stated above for the case $s=0$ (see $[\mathbf{R}$, p. 290]). However, standard regularity theory yields the estimate for general $s>0$. For a self-contained proof, one may refer to $[$ BD2].

In the following corollary, parts (i) and (ii) were explicitly stated in $[\mathbf{R}]$; the commutation relations follow from the definitions; the other parts follow from the hypoelliptic estimates by standard arguments.

Corollary 2.6.6 (Rumin). Let $\left(M^{2 n+1}, \eta\right)$ be a compact contact manifold with adapted metric $g$ and the associated complex $\left(\mathcal{R}^{*}, d_{R}\right)$; let $\Delta_{R}$ be the associated Laplacian.

(i) The cohomology of the complex is finite-dimensional and represented by $\Delta_{R}$-harmonic forms.

(ii) There exist operators $G_{R}, H_{R}: \mathcal{R}^{k} \rightarrow \mathcal{R}^{k}$ such that

$$
I d=G_{R} \Delta_{R}+H_{R}=\Delta_{R} G_{R}+H_{R},
$$

inducing the orthogonal decompositions:

$$
\mathcal{R}^{k}=\operatorname{ker} \Delta_{R} \oplus \text { range } \Delta_{R}=\operatorname{ker} \Delta_{R} \oplus\left(\text { range } d_{R} \oplus \text { range } \delta_{R}\right) .
$$

In particular, each $\alpha \in \mathcal{R}^{k}$ has a Hodge decomposition

$\alpha= \begin{cases}H_{R}(\alpha) \oplus(n-k) G_{R} d_{R} \delta_{R}(\alpha) \oplus(n-k+1) G_{R} \delta_{R} d_{R}(\alpha), & \text { for } k<n, \\ H_{R}(\alpha) \oplus G_{R}\left(d_{R} \delta_{R}\right)^{2}(\alpha) \oplus G_{R} D_{R}^{*} D_{R}(\alpha), & \text { for } k=n .\end{cases}$

(iii) The following commutation relations are satisfied:

For $P$ any of the operators $d_{R}, \delta_{R}, D_{R}$ or $D_{R}^{*}$,

$$
P H_{R}=H_{R} P=0 \text {; }
$$


for $\alpha \in \mathcal{R}^{k}$

$$
d_{R} G_{R}(\alpha)= \begin{cases}\left(\frac{n-k-1}{n-k+1}\right) G_{R} d_{R}(\alpha), & \text { for } k \leq n-2, \\ \frac{1}{2} G_{R}\left(d_{R} \delta_{R} d_{R}\right)(\alpha), & \text { for } k=n-1,\end{cases}
$$

and

$$
\delta_{R} G_{R}(\alpha)=\left(\frac{n-k+2}{n-k}\right) G_{R} \delta_{R}(\alpha), \quad \text { for } k \leq n-1 .
$$

(iv) For $\alpha \in \mathcal{R}^{k}, \begin{cases}\left\|G_{R} \alpha\right\|_{s+2} \leq c_{s}\|\alpha\|_{s} & \text { for } k \neq n,(n+1) \\ \left\|G_{R} \alpha\right\|_{s+4} \leq c_{s}\|\alpha\|_{s} & \text { for } k=n,(n+1)\end{cases}$ and $\left\|H_{R}(\alpha)\right\|_{s} \leq c_{s}\|\alpha\|_{s}$.

Moreover, since the space of harmonic forms is finite-dimensional, $\|\alpha\|_{s} \leq c_{s}\|\alpha\|_{0}$ for all $\alpha \in \operatorname{ker} \Delta_{R}$. In particular, $H_{R}(\alpha)$ is of class $C^{\infty}$ for all $\alpha \in \Gamma^{s}\left(R^{k}\right)$.

2.7. Characterization of contact vector fields. In this section, we present a characterization of the closed subspace $\Gamma_{\text {cont }}^{s}(T M) \subset \Gamma^{s}(T M)$ of $\Gamma^{s}$ contact vector fields in terms of the Hodge decomposition of the Rumin complex. We use this characterization in Section 4 to give a parameterization of the space of contact diffeomorphisms near the identity by contact vector fields near 0 .

We begin by recalling a few well-known facts about contact vector fields. Recall that a smooth vector field $X$ is called a contact vector field if and only if $\mathcal{L}_{X}(\eta)=0 \bmod \eta$ (or, equivalently, $\pi_{H}\left(\mathcal{L}_{X}(\eta)\right)=0$ ). Write $X$ in the form $X=X^{0} T+X_{H}$, where $X_{H} \in H$ and $T$ is the Reeb vector field, and use the identity

$$
\left.\left.\left.\mathcal{L}_{X}(\eta)=X\right\lrcorner d \eta+d(X\lrcorner \eta\right)=X_{H}\right\lrcorner d \eta+d X^{0}
$$

to conclude that $X$ is a contact vector field if and only if it satisfies the standard identity

$$
\left.-X_{H}\right\lrcorner d \eta+T\left(X^{0}\right) \eta=d X^{0} .
$$

Recalling that $R^{1}=H^{*}$ and $d_{R} f=\pi_{H}(d f)$, for $f \in C^{\infty}(M)=\mathcal{R}^{0}$, we can express this characterization in terms of the Rumin complex as follows: $X$ is a contact vector field if and only if it satisfies the identity

$$
\left.d_{R} X^{0}=-X_{H}\right\lrcorner d \eta
$$

Next observe that $d \eta$ defines a vector bundle map

$$
\left.()^{b}: T M \rightarrow H^{*}: X \mapsto X^{b}=X\right\lrcorner d \eta,
$$


whose restriction to $H \subset T M$ is an isomorphism between the contact distribution and its dual space, and let

$$
()^{\sharp}: H^{*} \rightarrow H: \phi \mapsto \phi^{\sharp}
$$

denote its inverse. The map

$$
C^{\infty}(M, \mathbb{R}) \rightarrow C_{\text {cont }}^{\infty}: g \mapsto X_{g}=g T-\left(d_{R} g\right)^{\sharp}
$$

is an isomorphism between the space of smooth functions on $M$ and the space of smooth contact vector fields. This map then extends to an isomorphism between the weighted spaces:

$$
\Gamma^{s+1}(M) \rightarrow \Gamma_{\text {cont }}^{s}(T M): g \mapsto X_{g}=g T-\left(d_{R} g\right)^{\sharp} .
$$

The only new result here is the gain of one derivative in the Folland-Stein spaces, which follows easily from the two inclusions $g \in \Gamma^{s}$ and $d_{R} g \in \Gamma^{s}$.

We can now express the condition for $X$ to be a contact vector field in terms of the harmonic decomposition. Note, in particular, the additional regularity in $X^{0}$, the Reeb component of $X$. (The restriction $s \geq 2 n+4$ below ensures that $X$ is of class $C^{1}$.)

Lemma 2.7.3. A vector field $X \in \Gamma^{s}(T M), s \geq 2 n+4$, is contact if and only if it satisfies each of the following three conditions

$$
\begin{aligned}
& \left.X^{0}=H_{R}\left(X^{0}\right)-(n+1) G_{R} \delta_{R}(X\lrcorner d \eta\right), \\
& \left.H_{R}(X\lrcorner d \eta\right)=0, \\
& \left\{\begin{array}{l}
\left.d_{R}(X\lrcorner d \eta\right)=0, \quad \text { for } n>1, \\
\left.D_{R}(X\lrcorner d \eta\right)=0, \quad \text { for } n=1 .
\end{array}\right.
\end{aligned}
$$

Moreover, if $X \in \Gamma^{s}(T M)$ is a contact vector field, then $X^{0} \in \Gamma^{s+1}(M)$.

Proof. Suppose that $X \in \Gamma^{s}(T M)$. Applying the Hodge decomposition to equation (2.7.1) shows that $X$ is a contact vector field if and only if it satisfies each of the three conditions

$$
\begin{aligned}
& \left.\left.H_{R}\left(d_{R} X^{0}+X\right\lrcorner d \eta\right)=0, \quad \delta_{R}\left(d_{R} X^{0}+X\right\lrcorner d \eta\right)=0, \text { and } \\
& \begin{cases}\left.d_{R}\left(d_{R} X^{0}+X\right\lrcorner d \eta\right)=0 & \text { if } n>1, \\
\left.D_{R}\left(d_{R} X^{0}+X\right\lrcorner d \eta\right)=0 & \text { if } n=1 .\end{cases}
\end{aligned}
$$

The middle equation is equivalent to

$$
\left.\left.G_{R} \delta_{R}\left(d_{R} X^{0}+X\right\lrcorner d \eta\right)=\frac{1}{(n+1)}\left(G_{R} \Delta_{R} X^{0}\right)+G_{R} \delta_{R}(X\lrcorner d \eta\right)=0,
$$

from which the conditions (2.7.3) follow. Finally, suppose that $X \in \Gamma^{s}(T M)$ is a contact vector field. By (a) and Corollary 2.6.6, the function $X^{0}$ is an element of $\Gamma^{s+1}(M)$. 


\section{The topological group of contact diffeomorphisms}

Let $\mathcal{D}^{s}(M) \subset \Gamma^{s}(M, M), s \geq 2 n+4$ denote the subspace of $\Gamma^{s}$ diffeomorphisms of $M$. It is well known that the space of $C^{1}$-diffeomorphisms is an open subset of the space $C^{1}(M, M)$ of $C^{1}$-maps. Moreover, since $s \geq 2 n+4$, there is a continuous inclusion $\Gamma^{s}(M, M) \subset C^{1}(M, M)$. It follows that $\mathcal{D}^{s}(M)$ is an open subset of $\Gamma^{s}(M, M)$. It is, therefore, a smooth infinite-dimensional manifold; but it is not a group because $\mathcal{D}^{s}(M)$ is not closed under composition (see Remark 2.1.1).

Let $\mathcal{D}_{\text {cont }}^{\infty}(M) \subset \mathcal{D}^{s}(M)$ denote the subspace of $C^{\infty}$ contact diffeomorphisms. By definition, the space of $\Gamma^{s}$ contact diffeomorphisms of $M$ is the closed subspace $\mathcal{D}_{\text {cont }}^{s}(M):=\overline{\mathcal{D}_{\text {cont }}^{\infty}(M)} \subset \mathcal{D}^{s}(M)$. We show in this section that $\mathcal{D}_{\text {cont }}^{s}(M)$ is closed under composition and inversion and that both operations are continuous. Consequently, $\mathcal{D}_{\text {cont }}^{s}(M)$ is a topological group for $s \geq 2 n+4$. In Section 4, we prove that $\mathcal{D}_{\text {cont }}^{s}(M)$ is a smooth Hilbert manifold (see Theorem 4.4.1). Our approach in this section and in the following section parallels the treatment of the full diffeomorphism group given by Ebin $[\mathbf{E}]$.

3.1. Continuity of composition. To prove that composition is continuous, it is sufficient to work locally. Consider open domains $D \Subset \mathbb{R}^{2 n+1}$ and $\widetilde{D} \Subset \mathbb{R}^{2 n+1}$. By the Sobolev lemma (see Remark 2.4.3), there is a continuous inclusion $\Gamma^{s}\left(D, \mathbb{R}^{2 n+1}\right) \subset C^{1}\left(D, \mathbb{R}^{2 n+1}\right)$. Consequently, the topological subspace $\mathcal{D}_{\text {cont }}^{s}(D, \tilde{D})$ of $C^{1}$ contact diffeomorphisms $f$ with $f(\bar{D}) \subset \widetilde{D}$ is well defined.

Proposition 3.1.1. Let $s \geq 2 n+4$, and let $f \in \mathcal{D}_{\text {cont }}^{s}(D, \tilde{D})$ and $g \in$ $\Gamma^{k}\left(\widetilde{D}, \mathbb{R}^{m}\right)$ for $k \leq s$. Let $D^{\prime} \Subset D$ be an open set. Then the restriction to $D^{\prime}$ of the composition $g \circ f$ is an element of $\Gamma^{k}\left(D^{\prime}, \mathbb{R}^{m}\right)$. Moreover, the map

$$
\mu: \mathcal{D}_{\text {cont }}^{s}(D, \tilde{D}) \times \Gamma^{k}\left(\widetilde{D}, \mathbb{R}^{m}\right) \rightarrow \Gamma^{k}\left(D^{\prime}, \mathbb{R}^{m}\right):(f, g) \mapsto g \circ f
$$

is continuous.

Proof. Our proof mimics the proof of Ebin [E, Lemma 3.1] in the case of diffeomorphisms of a manifold. It proceeds by induction on $k$.

For $k=0$, we first note that $\|g \circ f\|_{D^{\prime}, 0}<\infty$ for any $D^{\prime} \Subset D$. Since $f$ is a $C^{1}$ diffeomorphism on $D$, its Jacobian determinant $J f$ is continuous and bounded below on $\bar{D}^{\prime}$ by a positive constant; and (by the change of variables formula for integration)

$$
\|g \circ f\|_{D^{\prime}, 0}=\int_{D^{\prime}}(g \circ f)^{2} d V_{0}=\int_{f\left(D^{\prime}\right)} g^{2}\left(1 / J f \circ f^{-1}\right) d V_{0}<\infty .
$$

To prove continuity at $(f, g)$, choose $\epsilon>0$. We will show that $\| g^{\prime} \circ f^{\prime}-g \circ$ $f \|_{D^{\prime}, 0}^{2}<4 \epsilon$ for $\left(f^{\prime}, g^{\prime}\right)$ sufficiently near $(f, g)$. To see this, choose $\delta>0$ such 
that $\max _{\overline{D^{\prime}}}\left(1 / J\left(f^{\prime}\right), 1 / J(f)\right)<\epsilon / \delta$ whenever $\left\|f^{\prime}-f\right\|_{D, s}<\delta$. Also choose a smooth function $g^{\infty}$ on the closure of $\widetilde{D}$ such that $\left\|g-g^{\infty}\right\|_{\widetilde{D}, 0}^{2}<\delta$, and set

$$
M=\left(\max _{x \in \widetilde{D}} \sum_{i=1}^{2 n+1}\left|\frac{\partial g^{\infty}(x)}{\partial x^{i}}\right|^{2}\right) .
$$

Then

$$
\begin{aligned}
\left\|g \circ f-g^{\prime} \circ f^{\prime}\right\|_{D^{\prime}, 0}^{2} \leq & \left\|g \circ f-g^{\infty} \circ f\right\|_{D^{\prime}, 0}^{2}+\left\|g^{\infty} \circ f-g^{\infty} \circ f^{\prime}\right\|_{D^{\prime}, 0}^{2} \\
& +\left\|g^{\infty} \circ f^{\prime}-g \circ f^{\prime}\right\|_{D^{\prime}, 0}^{2}+\left\|g \circ f^{\prime}-g^{\prime} \circ f^{\prime}\right\|_{D^{\prime}, 0}^{2} \\
\leq & \frac{\epsilon}{\delta} \int_{\widetilde{D}}\left|g-g^{\infty}\right|^{2} d V_{0}+M \cdot\left\|f-f^{\prime}\right\|_{D, 0}^{2} \\
& +\frac{\epsilon}{\delta} \int_{\widetilde{D}}\left|g-g^{\infty}\right|^{2} d V_{0}+\frac{\epsilon}{\delta} \int_{\widetilde{D}}\left|g-g^{\prime}\right|^{2} d V_{0} \\
\leq & 2 \epsilon+M \cdot\left\|f-f^{\prime}\right\|_{D, 0}^{2}+\left(\frac{\epsilon}{\delta}\right)\left\|g-g^{\prime}\right\|_{\widetilde{D}, 0}^{2} .
\end{aligned}
$$

Now let $\delta^{\prime}=\min \left(\delta, \frac{\epsilon}{M}\right)$. Then the last line is bounded by $4 \epsilon$ provided that $f^{\prime}$ and $g^{\prime}$ satisfy the inequalities

$$
\left\|f-f^{\prime}\right\|_{D, 0}^{2}<\delta^{\prime} \quad \text { and } \quad\left\|g-g^{\prime}\right\|_{\widetilde{D}, 0}^{2}<\delta^{\prime} .
$$

Assume that for some $k \geq 0$ the proposition holds for all $D, D^{\prime} \Subset D$, and $\widetilde{D}$. We first show that $g \circ f$ is an element of $\Gamma^{k+1}\left(D^{\prime}\right), k+1 \leq s$, for all $g \in \Gamma^{k+1}\left(\widetilde{D}, \mathbb{R}^{m}\right)$. To do this, we need only to show that $X_{j}(g \circ f)$ is in $\Gamma^{k}\left(D^{\prime}\right)$ for $1 \leq j \leq 2 n$, where $X_{j}$ are the horizontal vector fields defined in Section 1.1. Begin by observing that since $f$ is a contact diffeomorphism, it's derivative $f_{*}$ respects the contact distribution on $\mathbb{R}^{2 n+1}$ :

$f_{H, *}: H_{x} \rightarrow H_{f(x)}: X_{j}(x) \mapsto f_{*}\left(X_{j}(x)\right)=\sum_{i=1}^{2 n+1} A_{j}^{i}(x) X_{i}(f(x)), \quad 1 \leq j \leq 2 n$,

where $A_{j}^{i} \in \Gamma^{s-1}(D, \mathbb{R})$ depend continuously on $f$. This permits us to compute as follows using the chain rule:

$$
X_{j}(g \circ f)=d g\left(f_{*}\left(X_{j}\right)\right)=\sum_{i=1}^{2 n} A_{j}^{i} \cdot X_{i}(g) \circ f .
$$

By the induction hypothesis, $X_{i}(g) \circ f \in \Gamma^{k}\left(D^{\prime \prime}\right)$ for any open set $D^{\prime \prime}$ such that $D^{\prime} \Subset D^{\prime \prime} \Subset D$. Since $s-1 \geq 2 n+3$, we can apply Lemma 2.2.1 to the products $A_{j}^{i} \cdot\left(X_{i}(g) \circ f\right)$ conclude that $A_{j}^{i} \cdot\left(X_{i}(g) \circ f\right) \in \Gamma^{k}\left(D^{\prime}\right)$, which in turn shows that $X_{j}(g \circ f) \in \Gamma^{k}\left(D^{\prime}\right)$. To complete the induction step, we have to prove continuity of composition. First note that if $g^{\prime}$ is near $g$ in $\Gamma^{k+1}(\widetilde{D})$ then $X_{i}\left(g^{\prime}\right)$ is near $X_{i}(g)$ in $\Gamma^{k}(\widetilde{D})$. Now choose a fixed open set $D^{\prime \prime}$ with 
$D^{\prime} \Subset D^{\prime \prime} \Subset D$. By the induction hypothesis, if $f^{\prime}$ is near $f$ in $\Gamma^{s}(D)$, then $X_{i}\left(g^{\prime}\right) \circ f^{\prime}$ is near $X_{i}(g) \circ f$ in $\Gamma^{k}\left(D^{\prime \prime}\right)$. But then by Lemma 2.2.1 it follows that $X_{j}\left(g^{\prime} \circ f^{\prime}\right)=\sum_{i=1}^{2 n}\left(A_{j}^{i}\right)^{\prime} \cdot\left(X_{i}\left(g^{\prime}\right) \circ f^{\prime}\right)$ is near $X_{j}(g \circ f)=\sum_{i=1}^{2 n} A_{j}^{i} \cdot\left(X_{i}(g) \circ f\right)$ in $\Gamma^{k}\left(D^{\prime}\right)$.

Corollary 3.1.4. Let $M$ be a compact contact manifold of dimension $2 n+1$, and let $N$ be a smooth manifold of dimension $m$. Then the composition map

$$
\mu: \mathcal{D}_{\text {cont }}^{s}(M) \times \Gamma^{k}(M, N) \rightarrow \Gamma^{k}(M, N):(F, G) \mapsto G \circ F
$$

is continuous for $2 n+4 \leq k \leq s$. In case $N=\mathbb{R}^{m}$, the map is continuous for $2 n+4 \leq s$ and $0 \leq k \leq s$.

Proof. In case $N=\mathbb{R}^{m}$, choose $s \geq 2 n+4$ and $0 \leq k \leq s$. Continuity of $\mu$ follows easily from the previous proposition. We next consider the case where $N$ is an arbitrary smooth manifold and the definition of $\Gamma^{k}(M, N)$ requires that $2 n+4 \leq k$. Fix $F \in \mathcal{D}_{\text {cont }}^{s}(M)$ and $G \in \Gamma^{k}(M, N)$. First note that the restriction $2 n+4 \leq k \leq s$ ensures that the spaces $\mathcal{D}_{\text {cont }}^{s}(M)$ and $\Gamma^{k}(M, N)$ are both well defined and that both $F$ and $G$ are of class $C^{1}$. Choose adapted atlases $\left\{\left(\phi_{\alpha}, U_{\alpha}, D_{\alpha}\right)\right\}$ and $\left\{\left(\widetilde{\phi}_{\alpha}, \widetilde{U}_{\alpha}, \widetilde{D}_{\alpha}\right)\right\}$ for $M$, and charts $\left\{\psi_{\alpha}, V_{\alpha}, B_{\alpha}\right\}$ for $N$, such that for all $\alpha$,

$$
F\left(\overline{U_{\alpha}}\right) \subset \widetilde{U}_{\alpha}, F_{\alpha}\left(\overline{D_{\alpha}}\right) \subset \widetilde{D}_{\alpha}, \quad \text { and } \quad G\left(\overline{\widetilde{U}_{\alpha}}\right) \subset V_{\alpha},
$$

where $F_{\alpha}=\widetilde{\phi}_{\alpha} \circ F \circ \phi_{\alpha}^{-1} \in \Gamma^{s}\left(U_{\alpha}, \widetilde{U}_{\alpha}\right)$ and $G_{\alpha}=\psi_{\alpha} \circ G \circ \widetilde{\phi}_{\alpha}^{-1} \in \Gamma^{k}\left(\widetilde{U}_{\alpha}, \mathbb{R}^{m}\right)$. Set $H_{\alpha}=G_{\alpha} \circ F_{\alpha}\left(=\psi_{\alpha} \circ G \circ F \circ \phi_{\alpha}^{-1}\right)$. By Proposition 3.1.1, $H_{\alpha} \in \Gamma^{k}\left(\widetilde{U}_{\alpha}, \mathbb{R}^{m}\right)$ for all $\alpha$, showing that $G \circ F$ is an element of $\Gamma^{k}(M, N)$. To prove continuity of $\mu$, consider the open neighbourhoods of $F, G$, and $H=G \circ F=\mu(F, G)$

$$
\begin{aligned}
& O\left(F, \epsilon,\left\{U_{\alpha}\right\}\right) \\
& \quad=\left\{F^{\prime} \in \mathcal{D}_{\text {cont }}^{s}(M): F^{\prime}\left(\overline{U_{\alpha}}\right) \subset \widetilde{U}_{\alpha} \text { for all } \alpha, \max _{\alpha}\left\|F_{\alpha}^{\prime}-F_{\alpha}\right\|_{U_{\sigma}, s}<\epsilon\right\}, \\
& O\left(G, \epsilon,\left\{\widetilde{U}_{\alpha}\right\}\right) \\
& \quad=\left\{G^{\prime} \in \Gamma^{k}(M, N): G^{\prime}\left(\overline{\widetilde{U}_{\alpha}}\right) \subset V_{\alpha} \text { for all } \alpha, \max _{\alpha}\left\|G_{\alpha}^{\prime}-G_{\alpha}\right\|_{\widetilde{U}_{\sigma}, k}<\epsilon\right\}, \\
& O\left(H, \epsilon,\left\{U_{\alpha}\right\}\right) \\
& \quad=\left\{H^{\prime} \in \Gamma^{k}(M, N): G^{\prime}\left(\overline{U_{\alpha}}\right) \subset V_{\alpha} \text { for all } \alpha, \max _{\alpha}\left\|H_{\alpha}^{\prime}-H_{\alpha}\right\|_{U_{\alpha}, k}<\epsilon\right\} .
\end{aligned}
$$

By definition of the topology of $\Gamma^{k}(M, N)$, every open neighbourhood of $H$ contains a set of the form $O\left(H, \epsilon,\left\{U_{\alpha}\right\}\right)$ for sufficiently small $\epsilon$. Moreover, by Proposition 3.1.1, for every $\epsilon>0$ there exists a $\delta>0$ such that

$$
H^{\prime}=G^{\prime} \circ F^{\prime} \in O\left(H, \epsilon,\left\{U_{\alpha}\right\}\right)
$$


for all $F^{\prime} \in O\left(F, \delta,\left\{U_{\alpha}\right\}\right)$ and $G^{\prime} \in O\left(G, \delta,\left\{\widetilde{U}_{\alpha}\right\}\right)$. Therefore,

$$
\mu\left(O\left(F, \delta,\left\{D_{\alpha}\right\}\right) \times O\left(G, \delta,\left\{\widetilde{D}_{\alpha}\right\}\right)\right) \subset O\left(G \circ F, \epsilon,\left\{U_{\alpha}\right\}\right) .
$$

This completes the proof of continuity of $\mu$.

3.2. Continuity of inversion. The proof of continuity of inversion relies on the next lemma.

Lemma 3.2.1. Let $f \in \mathcal{D}_{\text {cont }}^{s}(D, \tilde{D}), s \geq 2 n+4$ be a contact diffeomorphism with $f(D) \Subset \widetilde{D}$, and let $\widetilde{D}^{\prime} \Subset f(D)$. Then $f^{-1} \in \mathcal{D}_{\text {cont }}^{s}(M)\left(\widetilde{D}^{\prime}, D\right)$. Moreover, the map $\iota: f^{\prime} \mapsto f^{\prime-1}, f^{\prime} \in\left\{g \in \mathcal{D}_{\text {cont }}^{s}(D, \widetilde{D}): \widetilde{D}^{\prime} \subset g(D)\right\}$ is continuous at $f^{\prime}=f$.

Proof. Since $s \geq 2 n+4, f$ is a $C^{1}$ contact diffeomorphism on every compact subset of $D$. Hence, $f^{-1}$ is a $C^{1}$ contact diffeomorphism on every open, compactly contained, subset of $f(D)$. Let $A: D \rightarrow G L(2 n)$ be the matrix valued function defined by $A_{j}^{i}$, where $A_{j}^{i} \in \Gamma^{s-1}(D)$ are defined as in the proof of Proposition 3.1.1. Because the process of inverting $A$ only involves multiplication, addition, and division of functions in $\Gamma^{s-1}$, and because $s-1>2 n+3$, we can invoke Lemmas 2.2.1 and 2.2.2 to conclude that $A^{-1} \in \Gamma^{s-1}\left(D^{\prime}, G L(2 n)\right)$ for all $D^{\prime} \Subset D$.

Next observe that equation (3.1.3) with $g$ replaced by $g \circ f^{-1}$ assumes the form

$$
X_{j}(g)=\sum_{i=1}^{2 n} A_{j}^{i} \cdot X_{i}\left(g \circ f^{-1}\right) \circ f .
$$

Multiplying by $B=A^{-1}$ and composing with $f^{-1}$ then yields the formula

$$
X_{k}\left(g \circ f^{-1}\right)=\sum_{j}\left(B_{k}^{j} \cdot X_{j}(g)\right) \circ f^{-1} .
$$

To show that $f^{-1} \in \Gamma^{s}\left(\widetilde{D}^{\prime}, \mathbb{R}^{2 n+1}\right)$ for every open set $\widetilde{D}^{\prime} \Subset f(D)$, it suffices to show that $X_{I}\left(f^{-1}\right) \in \Gamma^{s-k}\left(\widetilde{D}^{\prime}\right)$ for every multi-index $I$ with $|I|=k$ (see Section 1.1). Following the argument on [E, page 17], we proceed by induction on $k$ to show that, for all $I$ with $|I|=k$,

$$
X_{I}\left(f^{-1}\right)=g_{I} \circ f^{-1} \text { with } g_{I} \in \Gamma^{s-k} .
$$

To see that (3.2.3) holds for $k=1$, let $g=i d_{\mathbb{R}^{2 n+1}}$ in (3.2.2) to get

$$
X_{i}\left(f^{-1}\right)=\left(\sum_{j}\left(B_{i}^{j} \cdot X_{j}\left(i d_{\mathbb{R}^{2 n+1}}\right)\right) \circ f^{-1}:=g_{i} \circ f^{-1},\right.
$$


and recall that $B \in \Gamma^{s-1}$ to conclude that $g_{i} \in \Gamma^{s-1}$. Now assume that (3.2.3) holds for $s>k>0$, and let $I=(i, J)$, for $J$ a multi-index with $|J|=k$. Then applying (3.2.2) gives

$$
X_{I}\left(f^{-1}\right)=X_{i}\left(g_{J} \circ f^{-1}\right)=\left(\sum_{j}\left(B_{i}^{j} \cdot X_{j}\left(g_{J}\right)\right)\right) \circ f^{-1}:=g_{I} \circ f^{-1} .
$$

Since $B_{i}^{j} \in \Gamma^{s-1}, s-1 \geq 2 n+3$, and $X_{j}\left(g_{J}\right) \in \Gamma^{s-k-1}$, we can invoke Lemma 2.2.1 to conclude that $X_{I}\left(f^{-1}\right)$ is of the form $g_{I} \circ f^{-1}$, for $g_{I} \in$ $\Gamma^{s-k-1}$. This completes the induction step. We now know that $X_{I}\left(f^{-1}\right)=$ $g_{I} \circ f^{-1}$ with $g_{I} \in \Gamma^{s-k} \subset \Gamma^{0}$, for all $I$ with $|I| \leq s$. But since $f^{-1}$ is of class $C^{1}$, the composition $X_{I}\left(f^{-1}\right)=g_{I} \circ f^{-1}$ is also in $\Gamma^{0}$ for all $I$ with $|I| \leq s$. Hence, $f^{-1}$ is in $\Gamma^{s}\left(\widetilde{D}^{\prime}, D\right)$ for all $\widetilde{D}^{\prime} \Subset f(D)$.

To prove continuity of the map $\iota: f^{\prime} \mapsto f^{\prime-1}$, we first show by finite induction that the map

$$
f^{\prime} \mapsto g_{I}^{\prime}:=X_{I}\left(f^{\prime-1}\right) \circ f^{\prime} \in \Gamma^{0}\left(\widetilde{D}^{\prime}, D\right)
$$

depends continuously on $f^{\prime} \in \mathcal{D}_{\text {cont }}^{s}$ for all $I$ with $|I| \leq s$. Let $k=1$, and note that by definition of $A$ (see equation (3.1.2)), the assignment $f^{\prime} \mapsto A^{\prime} \mapsto$ $B^{\prime}=A^{\prime-1} \in \Gamma^{s-1}$ depends continuously on $f^{\prime} \in \mathcal{D}_{\text {cont }}^{s}$. Hence, $g_{i}^{\prime} \in \Gamma^{s-1}$ depends continuously on $f^{\prime} \in \mathcal{D}_{\text {cont }}^{s}$. Now assume that $f^{\prime} \mapsto g_{J}^{\prime} \in \Gamma^{s-k}$ depends continuously on $f^{\prime}$ for all $J,|J|=k$. Set $I=(i, J)$. Then $X_{i}\left(g_{J}^{\prime}\right) \in$ $\Gamma^{s-k-1}$ depends continuously on $f^{\prime}$. Hence by (3.2.4), $g_{I}^{\prime} \in \Gamma^{s-k-1}$ depends continuously on $f^{\prime}$, completing the induction step.

Thus, for any multi-index $I$ with $|I| \leq s$,

$$
\begin{aligned}
& \left\|X_{I}\left(f^{\prime-1}-f^{-1}\right)\right\|_{\widetilde{D}^{\prime}, 0} \\
& \left.\quad=\| g_{I}^{\prime} \circ f^{\prime-1}-g_{I} \circ f^{-1}\right) \|_{\widetilde{D}^{\prime}, 0} \\
& \quad \leq\left\|g_{I}^{\prime} \circ f^{\prime-1}-g_{I} \circ f^{\prime-1}\right\|_{\widetilde{D}^{\prime}, 0}+\left\|g_{I} \circ f^{\prime-1}-g_{I} \circ f^{-1}\right\|_{\widetilde{D}^{\prime}, 0} .
\end{aligned}
$$

Because the map $f^{\prime} \mapsto f^{\prime-1}$ is continuous in the $C^{1}$-topology, by making making $\left\|f^{\prime}-f\right\|_{\widetilde{D}^{\prime}, s}$ sufficiently small, we ensure that $\left\|X_{I}\left(f^{\prime-1}-f^{-1}\right)\right\|_{\widetilde{D}^{\prime}, 0}$ is arbitrarily small for all $I$ with $|I| \leq s$. This concludes the proof of continuity of $\iota$.

Theorem 3.2.5. Let $s \geq 2 n+4$. Then $\mathcal{D}_{\text {cont }}^{s}(M)$ is a topological group with group multiplication

$$
\mu: \mathcal{D}_{\text {cont }}^{s}(M) \times \mathcal{D}_{\text {cont }}^{s}(M) \rightarrow \mathcal{D}_{\text {cont }}^{s}(M):(F, G) \mapsto G \circ F
$$

and group inverse

$$
\iota: \mathcal{D}_{\text {cont }}^{s}(M) \rightarrow \mathcal{D}_{\text {cont }}^{s}(M): F \mapsto F^{-1} .
$$


Proof. Continuity of $\mu$ is contained in Corollary 3.1.4. Continuity of $\iota$ follows from Lemma 3.2.1 by an argument similar to the one used in the proof of Corollary 3.1.4. In brief, for fixed $F$, choose adapted atlases $\left\{\left(\phi_{\alpha}, U_{\alpha}, D_{\alpha}\right)\right\}$, and $\left\{\left(\phi_{\alpha}^{\prime}, U_{\alpha}^{\prime}, D_{\alpha}^{\prime}\right)\right\}$ such that $D_{\alpha}^{\prime} \subset F_{\alpha}\left(D_{\alpha}\right)$ for all $\alpha$. Then by Lemma 3.2.1, for all $\epsilon>0$ there there is a $\delta>0$ such that for all $G \in O(F, \delta)$ we have $G^{-1} \in O\left(F^{-1}, \epsilon\right)$.

\section{The smooth manifold of contact diffeomorphisms}

In this section, we obtain a local coordinate chart for the set of contact diffeomorphisms in a neighbourhood of the identity. As a corollary, we show that for $s \geq 2 n+4$, the topological manifold $\mathcal{D}_{\text {cont }}^{s}(M)$ is a smooth submanifold of the smooth manifold $\mathcal{D}^{s}(M)$ of $\Gamma^{s}$ diffeomorphisms of $M$.

4.1. The smooth manifold of contact diffeomorphisms. We begin by constructing a smooth atlas for $\mathcal{D}^{s}(M)$. Our construction is based on the following well known parameterization of smooth diffeomorphisms near the identity diffeomorphism by smooth vector fields. Fix a $C^{\infty}$ metric adapted to the contact structure (see Section 1.1), and let exp : $T M \rightarrow M$ denote its exponential map. Recall that exp is the $C^{\infty}$ map defined by the formula

$$
\exp (X):=\gamma_{x, X}(1)
$$

where $\gamma_{x, X}: \mathbb{R} \rightarrow M$ is the unique geodesic curve with $\gamma_{x, X}(0)=x, \gamma^{\prime}(0)=$ $X \in T_{x} M$. Also recall that for $|X|$ sufficiently small, $|X|$ is equal to the Riemannian distance between $x$ and $\exp (X)$. Next consider the map $\chi$ from the space of $C^{1}$-vector fields to the space of $C^{1}$-maps

$$
\chi: C^{1}(T M) \rightarrow C^{1}(M, M): X \mapsto F_{X},
$$

where $F_{X}$ is the $C^{1}$ map defined by composition

$$
F_{X}: M \stackrel{X}{\longrightarrow} T M \stackrel{\exp }{\longrightarrow} M .
$$

By compactness of $M$, there is a number $r>0$ such that any two points at distance less then $r$ apart are joined by a unique length minimizing geodesic. Let $B_{r} M \subset T M$ denote the bundle over $M$ of tangent vectors of length less than $r$. It is a well-known theorem in Riemannian geometry that $\chi$ restricts to a diffeomorphism between the space $C^{1}\left(B_{r} M\right)$ of $C^{1}$-vector fields of length less than $r$ and the open set

$$
\left\{F \in C^{1}(M, M): \operatorname{dist}_{M}(x, F(x))<r, \text { for all } x \in M\right\},
$$

where $\operatorname{dist}_{M}$ denotes Riemannian distance.

Proposition 4.1.2. For $s \geq 2 n+4$, the map $\chi$ restricts to a smooth map

$$
\chi^{s}: \Gamma^{s}(T M) \rightarrow \Gamma^{s}(M, M): X \mapsto F_{X}=\exp \circ X
$$


on the space of $\Gamma^{s}$-vector fields. Moreover, there is an open neighbourhood $\mathcal{U} \subset \Gamma^{2 n+4}(T M)$ of the zero section, such that for all $s \geq 2 n+4, \chi^{s}$ restricts to a diffeomorphism

$$
\chi^{s}: \mathcal{U}^{s} \equiv \mathcal{U} \cap \Gamma^{s}(T M) \rightarrow \mathcal{D}^{s}(M)
$$

between $\mathcal{U}^{s}$ and a neighbourhood of the identity $i d_{M} \in \mathcal{D}^{s}(M)$.

Proof. First observe that a map $F: M \rightarrow M$ can be viewed as a section of the trivial fibre bundle $\pi_{M}: M \times M \rightarrow M:(x, y) \mapsto x$ and that exp defines a smooth map of fibre bundles

$$
\widetilde{\exp }: T M \rightarrow M \times M: X \mapsto(\pi(X), \exp (X)),
$$

where $\pi: T M \rightarrow M$ is projection onto the base point. Smoothness of $\chi^{s}$ then follows from Proposition 2.3.5. Let $U_{r} M=\left\{(x, y) \in M \times M\right.$ : $\operatorname{dist}_{M}(x, y)<$ $r\}$. Then $\widetilde{\exp }: B_{r} M \rightarrow U_{r} M$ is a smooth fibre bundle isomorphism. By Proposition 2.4.2, the restriction of $\chi$ to $\Gamma^{s}$-spaces

$$
\chi^{s}: \Gamma^{s}\left(B_{r} M\right) \rightarrow \Gamma^{s}\left(U_{r} M\right)
$$

is therefore a diffeomorphism. To complete the proof, let $\mathcal{U}$ denote the preimage of the open set $\Gamma^{2 n+4}\left(U_{r} M\right) \cap \mathcal{D}^{2 n+4}(M)$ under $\chi^{2 n+4}$.

Remark 4.1.3. We can use $\chi^{s}$ to construct a smooth atlas for smooth manifold $\mathcal{D}^{s}(M)$. Let $G$ be a smooth diffeomorphism of $M$. By Corollary 3.1.4, that composition on the left with $G$ gives a smooth diffeomorphism of $\mathcal{D}^{s}(M)$. Consequently, the map

$$
\chi_{G}^{s}:=L_{G} \circ \chi^{s}: \mathcal{U}^{s} \rightarrow \mathcal{D}^{s}(M): X \mapsto G \circ F_{X}
$$

is a local diffeomorphism. Since the set of $C^{\infty}$ diffeomorphisms of $M$ is dense in $\mathcal{D}^{s}(M)$, letting $G$ range over all diffeomorphisms gives a smooth atlas. Since composition of smooth maps is smooth, smoothness of the transition functions is automatic.

Remark 4.1.4. We recall the standard construction of the tangent bundle $\pi_{\mathcal{D}(M)}: T \mathcal{D}^{s}(M) \rightarrow \mathcal{D}^{s}(M)$ (see [Ham, Pal] for background). To get a tangent vector to $\mathcal{D}^{s}(M)$ at $F_{0} \in \mathcal{D}^{s}(M)$, let $t \mapsto F_{t}$ be a smooth curve in $\mathcal{D}^{s}(M)$ passing through $F_{0}$. Then $F_{t}$ is a smooth family of $C^{1}$ diffeomorphisms, so we can differentiate pointwise with respect to $t$ to obtain the vector field

$$
X: M \rightarrow T M: x \mapsto \dot{F}_{0}(x) \in T M_{F_{0}(x)}
$$

over $F_{0}$, where we have used the notation $\dot{F}_{0}(x):=\left.\frac{d F_{t}(x)}{d t}\right|_{t=0}$. Conversely, given a vector field $X \in \Gamma^{s}(M, T M)$ with $\pi \circ X=F_{0}$, observe that for small $t$ the composition

$$
F_{t}: M \stackrel{t X}{\longrightarrow} T M \stackrel{\exp }{\longrightarrow} M
$$


is a smooth family in $\mathcal{D}^{s}(M)$ and that $X=\dot{F}_{0}$. An easy way to obtain the manifold structure on the total space $T \mathcal{D}^{s}(M)$ is to note that, by Corollary 2.4.5, composition with the projection map $\pi: T M \rightarrow M$ induces a smooth map $L_{\pi}^{s}: \Gamma^{s}(M, T M) \rightarrow \Gamma^{s}(M, M)$. Let $T \mathcal{D}^{s}(M)$ be the preimage of $\mathcal{D}^{s}(M)$ under $L_{\pi}^{s}$ and let $\pi_{\mathcal{D}(M)}=L_{\pi}^{s}$.

4.2. Characterization of contact diffeomorphisms. Recall that a diffeomorphism $F$ is a contact diffeomorphism if and only if the pullback $F^{*} \eta$ is a multiple of $\eta$. Since this condition is equivalent to the equation $\pi_{H}\left(F^{*} \eta\right)=0$, where $\pi_{H}: T^{*} M \rightarrow H^{*}$ is the quotient map, the space of $\Gamma^{s}$ contact diffeomorphisms near the identity is parameterized by the subspace

$$
\mathcal{V}^{s} \equiv\left\{X \in \mathcal{U}^{s}: \pi_{H}\left(F_{X}^{*} \eta\right)=0\right\} \subset \Gamma^{s}(T M) .
$$

It is convenient to view the equation $\pi_{H}\left(F_{X}^{*} \eta\right)=0$ in terms of the Rumin complex. Note that $R^{1} \equiv H^{*}$; hence $F_{X} \in \mathcal{U}^{s}$ is a contact diffeomorphism if and only if it satisfies the equation

$$
\pi_{R}\left(F_{X}^{*} \eta\right)=0
$$

This suggests studying the non-linear differential operator

$$
X \mapsto \pi_{R}\left(F_{X}^{*} \eta\right)
$$

in more detail. Our goal is to show that

$$
\pi_{R}\left(F_{X}^{*} \eta\right)=\pi_{R} \mathcal{L}_{X} \eta+\pi_{R} \circ Q_{\eta}(X),
$$

where $\mathcal{L}_{X}$ denotes Lie differentiation with respect to the vector field $X$ and $Q_{\eta}(X)$ is a smooth differential operator that vanishes to second order as $X \rightarrow 0$. Part (ii) of the next proposition shows that $X \mapsto \pi_{R}\left(F_{X}^{*} \eta\right)$ is a smooth differential operator of contact order 1; that it has the form of equation (4.2.2) is a corollary to Lemma 4.2.7.

Proposition 4.2.3. The following maps are smooth (non-linear) differential operators for all $s \geq 2 n+4$ :

(i) $\Gamma^{s}(T M) \rightarrow \Gamma^{s-2}\left(T^{*} M\right): X \mapsto F_{X}^{*} \eta$,

(ii) $\Gamma^{s}(T M) \rightarrow \Gamma^{s-1}\left(R^{1}\right): X \mapsto \pi_{R} F_{X}^{*} \eta$,

(iii) $\Gamma^{s}(T M) \rightarrow \Gamma^{s-1}\left(R^{2}\right): X \mapsto d_{R}\left(\pi_{R} F_{X}^{*} \eta\right)=\pi_{R}\left(d F_{X}^{*} \eta\right)=\pi_{R} F_{X}^{*}(d \eta)$, for $n>1$.

Proof. View $F_{X}: M \rightarrow M$ as a section of the trivial bundle $M \times M \rightarrow M$. Since $s \geq 2 n+4$, Example 2.5.9 parts (iv) and (v) apply to yield (i) and (ii). To prove (iii), apply Example 2.5.9 (v) to the smooth 2-form $d \eta$ to conclude that the map

$$
X \mapsto \pi_{H}\left(F_{X}^{*} d \eta\right)
$$


is a smooth differential operator of contact order 1 . Next observe that $\pi_{R}=$ $\tau \circ \pi_{H}$, where $\tau$ is the quotient map given by (2.6.1). Finally, since $d_{R} \beta=$ $\pi_{R}(d \beta)$, the composition

$$
X \mapsto \pi_{H}\left(F_{X}^{*}(d \eta)\right) \mapsto \tau\left(\pi_{H}\left(F_{X}^{*} d \eta\right)\right)=\pi_{R} F_{X}^{*}(d \eta)=\pi_{R} d\left(F_{X}^{*} \eta\right)
$$

is also a smooth differential operator of contact order 1 .

To show that $\pi_{H}\left(F_{X}^{*} \eta\right)$ has the form of equation (4.2.2), we work locally, choosing an adapted atlas $\phi_{\alpha}: U_{\alpha} \rightarrow \mathbb{R}^{2 n+1}$ for $M$ and a collection of open sets $W_{\alpha} \Subset U_{\alpha}$ covering $M$ as in Section 1.1. By compactness of $M$, there is a constant $c>0$ such that $\exp (x, X) \in U_{\alpha}$ for all $x \in \overline{W_{\alpha}}$, all $X \in T M_{x}$, with $|X|<c$, and all $\alpha$.

Let $X$ be a $C^{1}$ vector field with $|X|<c$. Fix a chart, say $\phi_{\alpha}$, and set $U=$ $U_{\alpha}$ and $W=W_{\alpha}$. To simplify notation, we adopt the Einstein summation conventions, letting Roman indices range from 1 to $2 n+1$. Then there exist smooth functions $B_{i j}^{k}(x, X)$ (locally defined) on $T M$ such that

$$
\exp ^{k}(x, X)=x^{k}+X^{k}+B_{i j}^{k}(x, X) X^{i} X^{j} .
$$

This follows simply from the second-order Taylor's formula with integral remainder for the exponential map. Indeed, for fixed $X \in T_{x} M$, let $\gamma(t)=$ $\exp (x, t X)$ be a geodesic. Then

$$
\gamma^{k}(1)=\gamma^{k}(0)+\dot{\gamma}^{k}(0)-\int_{0}^{1}(1-t) \Gamma_{i j}^{k}(\gamma(t)) \dot{\gamma}^{i}(t) \dot{\gamma}^{j}(t) d t
$$

where $\Gamma_{i j}^{k}$ are the Christoffel symbols, and we have used the geodesic equation $\ddot{\gamma}^{k}+\Gamma_{i j}^{k} \dot{\gamma}^{i} \dot{\gamma}^{j}=0$. Let $y=\exp (x, X)$. Since $\gamma(t)=\exp (x, t X)=y(x, t X)$, then

and this becomes

$$
\dot{\gamma}^{i}(t)=\dot{y}^{i}(x, t X)=\frac{\partial y^{i}(x, t X)}{\partial X^{j}} X^{j},
$$

$$
\begin{aligned}
\gamma^{k}(1)= & \gamma^{k}(0)+\dot{\gamma}^{k}(0)-\int_{0}^{1}(1-t) \Gamma_{a b}^{k}(\exp (x, t X)) \frac{\partial y^{a}}{\partial X^{i}}(x, t X) \\
& \times \frac{\partial y^{b}}{\partial X^{j}}(x, t X) X^{i} X^{j} d t
\end{aligned}
$$

when

$$
B_{i j}^{k}(x, X)=-\int_{0}^{1}(1-t) \Gamma_{a b}^{k}(\exp (x, t X)) \frac{\partial y^{a}}{\partial X^{i}}(x, t X) \frac{\partial y^{b}}{\partial X^{j}}(x, t X) d t
$$

Lemma 4.2.7. Let $\psi$ be a smooth $q$-form on $M$ and choose a coordinate patch $U=U^{\alpha}$, with $W=W_{\alpha} \Subset U$. Let $c>0$ be chosen so that $\exp (x, X) \in$ $U$ for all $x \in \bar{W}$ and all $X \in T_{x} M$ with $|X|<c$. Then there are (locally defined) smooth fibre bundle maps

$$
Q_{i j}^{1}:\left.\left.B M\right|_{W} \rightarrow \Lambda^{q} M\right|_{W} \quad \text { and } \quad Q_{i j}^{2}:\left.\left.B M\right|_{W} \rightarrow \Lambda^{q-1} M\right|_{W},
$$


where $B M=\{X \in T M:|X|<c\}$, such that for any $C^{1}$ vector field $X: M \rightarrow B M \subset T M$ the equation

$$
F_{X}^{*} \psi=\psi+\mathcal{L}_{X} \psi+Q_{i j}^{1}(X) X^{i} X^{j}+Q_{i j}^{2}(X) \wedge X^{i} d X^{j}
$$

is satisfied on all of $W$.

Proof. Begin with the special case of a 0 -form $u \in C^{\infty}(M, \mathbb{R})$. Then $F_{X}^{*} u(x)=u \circ \exp (x, X)$, and applying Taylor's formula with integral remainder to the function $f(t)=u\left(x+t X+t^{2} B_{i j}(x, t X) X^{i} X^{j}\right)$ and setting $t=1$ yields the formula

$$
(u \circ \exp )(x, X)=u(x)+\mathcal{L}_{X} u(x)+Q_{i j}(x, X) X^{i} X^{j},
$$

for $Q_{i j}(x, X)$ smooth functions on $\left.B M\right|_{W}$, such that $Q_{i j}=Q_{j i}$. Next consider the special case $\psi=d x^{k}$, and compute as follows, using what we have just proved:

$$
\begin{aligned}
F_{X}^{*}\left(d x^{k}\right) & =d\left(\exp _{X}^{*} x^{k}\right) \\
& =d\left(x^{k}+\mathcal{L}_{X}\left(x^{k}\right)+Q_{i j}(x, X) X^{i} X^{j}\right) \\
& =d x^{k}+\mathcal{L}_{X} d x^{k}+d\left(Q_{i j}(x, X) X^{i} X^{j}\right) \\
& =d x^{k}+\mathcal{L}_{X} d x^{k}+d\left(Q_{i j}(x, X)\right) X^{i} X^{j}+2 Q_{i j}(x, X) X^{i} d X^{j} \\
& =d x^{k}+\mathcal{L}_{X} d x^{k}+Q_{i j}^{1} X^{i} X^{j}+Q_{i j}^{2}(x, X) X^{i} d X^{j}
\end{aligned}
$$

for $Q_{i j}^{1}=\frac{\partial Q_{i j}}{\partial x^{k}} d x^{k}, Q_{i j}^{2}=\frac{\partial Q_{i k}}{\partial X^{j}} X^{k}+2 Q_{i j}=\frac{\partial\left(Q_{i k} X^{k}\right)}{\partial X^{j}}+Q_{i j}$. Because every $p$-form can be expressed as a linear combination of products of terms as above, the general result follows easily by induction.

Remark 4.2.8. Henceforth, we will use the notation

$$
Q_{\psi}(X):=F_{X}^{*}(\psi)-\psi-\mathcal{L}_{X} \psi
$$

to denote the non-linear part of the pull-back $F_{X}^{*} \psi$. The lemma states that in local coordinates

$$
Q_{\psi}(X)=Q_{i j}^{1}(X) X^{i} X^{j}+Q_{i j}^{2}(X) \wedge X^{i} d X^{j},
$$

where $Q_{i j}^{1}$ and $Q_{i j}^{2}$ are smooth functions on $\left.B M\right|_{W} \subset T M$, which depend on the smooth form $\psi$.

4.3. Parameterization of contact diffeomorphisms. The condition for $F_{X}$ to be a contact diffeomorphism is the vanishing of the one-form $F_{X}^{*} \eta \bmod$ $\eta$. Remark 4.2 .8 applied to $\psi=\eta$ and the identity $\pi_{R} \eta=0$ show that $F_{X}$ is a contact diffeomorphism if and only if

$$
\pi_{R} \mathcal{L}_{X} \eta+\pi_{R} Q_{\eta}(X)=0 .
$$


Since $Q_{\eta}(X)$ vanishes to second order at $X=0$, the linearization of this equation is

$$
\pi_{R} \mathcal{L}_{X} \eta=0
$$

i.e., the condition that $X$ be a contact vector field. This suggests using the implicit function theorem in Banach spaces to construct a parameterization of the space of contact diffeomorphisms near the identity by the space contact vector fields near zero.

We are going to construct a smooth map between Hilbert spaces of the form

$$
\Phi: \Gamma^{s}(T M) \rightarrow \Gamma^{s+1}(M) \times \mathcal{H}^{s}: X \mapsto g_{X} \oplus \gamma_{X},
$$

where $\mathcal{H}^{s}$ is a second Hilbert space (to be determined), such that

(i) $F_{X}$ is a contact diffeomorphism if and only if $\gamma_{X}=0$,

(ii) the derivative of $\Phi$ is invertible at the origin.

By the inverse function theorem, $\Phi$ is locally invertible and the map

$$
g \mapsto \chi^{s}\left(\Phi^{-1}(g, 0)\right)
$$

gives a smooth parameterization of the contact diffeomorphisms in $\mathcal{D}_{\text {cont }}^{s}(M)$ near the identity by real valued functions in $\Gamma^{s+1}(M)$ near zero.

A natural guess for the map $\Phi$ is

$$
X=X^{0} T+X_{H} \mapsto\left(X^{0}, \pi_{R} F_{X}^{*} \eta\right),
$$

for, as we have already observed, $F_{X}$ is contact if and only if $\pi_{R} F_{X}^{*} \eta=0$, and $X^{0}$ parameterizes contact vector fields (see Section 2.7). Unfortunately, this map is not invertible. Indeed, its linearization at the origin involves a differential operator that loses too many derivatives.

The trick to circumventing this difficulty is to exploit some hidden smoothness in the Hodge decomposition of one-forms in the Rumin complex. For smooth data, choose $\Phi$ to be of the form

$$
\Phi:\left\{\begin{aligned}
C^{\infty}(T M) & \rightarrow C^{\infty}(M, \mathbb{R}) \oplus \operatorname{range}\left(\delta_{R}\right) \oplus \operatorname{ker}\left(\delta_{R}\right) \\
& \subset C^{\infty}(M, \mathbb{R}) \oplus C^{\infty}(M, \mathbb{R}) \oplus \mathcal{R}^{1} \\
X & \mapsto g_{X} \oplus \alpha_{X} \oplus \omega_{X} .
\end{aligned}\right.
$$

When $n>1$, the Hodge theory shows that the projection $\pi_{R} \alpha \in \mathcal{R}^{1}$ of a general one-form $\alpha \in \Omega^{1}$ has the decomposition

$$
\begin{aligned}
\pi_{R} \alpha & =G_{R}\left\{(n-1) d_{R} \delta_{R}+n \delta_{R} d_{R}\right\} \pi_{R} \alpha+H_{R} \pi_{R} \alpha \\
& =(n-1) G_{R} d_{R} \delta_{R} \pi_{R} \alpha+n G_{R} \delta_{R} \pi_{R} d \alpha+H_{R} \pi_{R} \alpha .
\end{aligned}
$$

Applying the commutation relations of Corollary 2.6.6(iii), gives

$$
\pi_{R} \alpha=(n+1) d_{R} G_{R} \delta_{R} \pi_{R} \alpha+n G_{R} \delta_{R} \pi_{R} d \alpha+H_{R} \pi_{R} \alpha .
$$


This, together with the identity $d F_{X}^{*} \eta=F_{X}^{*} d \eta$ give the following decomposition of $\pi_{R} F_{X}^{*} \eta$ :

$$
\pi_{R} F_{X}^{*} \eta=(n+1) d_{R} G_{R} \delta_{R}\left(\pi_{R} F_{X}^{*} \eta\right)+n G_{R} \delta_{R}\left(\pi_{R} F_{X}^{*} d \eta\right)+H_{R}\left(\pi_{R} F_{X}^{*} \eta\right) .
$$

Referring now to our natural guess (4.3.1), we reassemble it using equation (2.7.3)(a) for $X^{0}$ and the identity (4.3.2) to define the map $\Phi$ by the formulas

$$
\left\{\begin{array}{l}
\left.g_{X}=-(n+1) G_{R} \delta_{R}\left(X_{H}\right\lrcorner d \eta\right)+H_{R}\left(X^{0}\right), \\
\alpha_{X}=(n+1) G_{R} \delta_{R}\left(\pi_{R} F_{X}^{*} \eta\right), \\
\omega_{X}=n G_{R} \delta_{R} d_{R}\left(\pi_{R} F_{X}^{*} \eta\right)+H_{R}\left(\pi_{R} F_{X}^{*} \eta\right) .
\end{array}\right.
$$

Indeed, we observe that $\pi_{R} F_{X}^{*} \eta=d_{R} \alpha_{X}+\omega_{X}$ by equation (4.3.2), and in the case where $X$ is a contact vector field, then $g_{X}=X_{0}$ by Lemma 2.7.3. In the case $n=1$, we have to adjust the map $\Phi$ to reflect the Hodge decomposition at $\mathcal{R}^{n}=\mathcal{R}^{1}$ :

$$
\pi_{R} F_{X}^{*} \eta=G_{R}\left(d_{R} \delta_{R}\right)^{2}\left(\pi_{R} F_{X}^{*} \eta\right)+G_{R} D_{R}^{*} D_{R}\left(\pi_{R} F_{X}^{*} \eta\right)+H_{R}\left(\pi_{R} F_{X}^{*} \eta\right) .
$$

Applying the commutation relation 2.6.6(iii) to the 0 -form $\delta_{R}\left(\pi_{R} F_{X}^{*} \eta\right.$ ), yields the identity

$$
\pi_{R} F_{X}^{*} \eta=2 d_{R} G_{R} \delta_{R}\left(\pi_{R} F_{X}^{*} \eta\right)+G_{R} D_{R}^{*} D_{R}\left(\pi_{R} F_{X}^{*} \eta\right)+H_{R}\left(\pi_{R} F_{X}^{*} \eta\right) .
$$

Because $n+1=2$, the formulas for $g, \alpha$, and $\omega$ become

$$
\left\{\begin{array}{l}
\left.g_{X}=-(n+1) G_{R} \delta_{R}\left(X_{H}\right\lrcorner d \eta\right)+H_{R}\left(X^{0}\right), \\
\alpha_{X}=(n+1) G_{R} \delta_{R}\left(\pi_{R} F_{X}^{*} \eta\right), \\
\omega_{X}=G_{R} D_{R}^{*} D_{R}\left(\pi_{R} F_{X}^{*} \eta\right)+H_{R}\left(\pi_{R} F_{X}^{*} \eta\right),
\end{array}\right.
$$

and only the formula for $\omega_{X}$ has changed.

Remark 4.3.5. Observe that by construction $\pi_{R} F_{X}^{*} \eta=d_{R} \alpha_{X}+\omega_{X}$. Since $d_{R}$ is injective on range $\left(\delta_{R}\right), \alpha_{X}=0$ if and only if $d_{R} \alpha_{X}=0$. Consequently, $F_{X}$ is a contact diffeomorphism if and only if $\gamma_{X}=\alpha_{X} \oplus \omega_{X}=0 \oplus 0$.

Remark 4.3.6. In the case $n=1$, the map $\Phi$ is, roughly speaking, the same as the map defined in $[\mathbf{B}]$; however, in that paper, the use of the complex Laplacian and complex operators necessitated an additional splitting into real and imaginary parts - roughly doubling the number of terms.

Proposition 4.3.7. Let $\Phi$ be the map defined above and let

$$
\mathcal{H}^{s}:=\Gamma^{s}\left(\operatorname{range}\left(\delta_{R}\right)\right) \oplus \Gamma^{s}\left(\operatorname{ker}\left(\delta_{R}\right)\right) \subset \Gamma^{s}(M) \oplus \Gamma^{s}\left(R^{1}\right) .
$$

Then for $s \geq 2 n+4$, the map $\Phi$ extends to a smooth map

$$
\Phi:\left\{\begin{array}{cl}
\Gamma^{s}(T M) & \rightarrow \Gamma^{s+1}(M) \oplus \mathcal{H}^{s} \\
X & \mapsto g_{X} \oplus \gamma_{X}:=g_{X} \oplus\left(\alpha_{X} \oplus \omega_{X}\right)
\end{array}\right.
$$


The linearization of $\Phi$ at the zero vector field is given by

$$
d \Phi(X)=\left\{\begin{aligned}
\{- & \left.\left.(n+1) G_{R} \delta_{R}\left(X_{H}\right\lrcorner d \eta\right)+H_{R}\left(X^{0}\right)\right\} \\
& \oplus\left\{(n+1) G_{R} \delta_{R}\left(d_{R} X^{0}+X_{H}\right\lrcorner d \eta\right) \oplus\left(n G_{R} \delta_{R} d_{R}\left(X_{H}\right\lrcorner d \eta\right) \\
& \left.\left.+H_{R}\left(X_{H}\right\rfloor d \eta\right)\right\}, \quad \text { for } n>1, \\
\{- & \left.\left.(n+1) G_{R} \delta_{R}\left(X_{H}\right\lrcorner d \eta\right)+H_{R}\left(X^{0}\right)\right\} \\
& \oplus\left\{(n+1) G_{R} \delta_{R}\left(d_{R} X^{0}+X_{H}\right\lrcorner d \eta\right) \oplus\left(G_{R} D_{R}^{*} D_{R}\left(X_{H}\right\lrcorner d \eta\right) \\
& \left.\left.\left.+H_{R}\left(X_{H}\right\lrcorner d \eta\right)\right)\right\}, \quad \text { for } n=1 .
\end{aligned}\right.
$$

Moreover, the linearization of $\Phi$ is invertible with inverse given by

$$
g \oplus(\alpha \oplus \omega) \mapsto(g+\alpha) T+\left(-d_{R} g+\omega\right)^{\sharp},
$$

where $\sharp$ is the isomorphism from horizontal one-forms to horizontal vector fields induced by the two-form $d \eta$.

The proof relies on two lemmas.

Lemma 4.3.8. Let $F \in \Gamma^{s}(M, M), s \geq 2 n+4$, be a diffeomorphism, and let $\beta \in \Omega^{k}$ be a smooth $k$-form, $k \leq n$. Then the form $\pi_{R} F^{*} \beta$ lies in $\Gamma^{s-1}\left(R^{k}\right)$.

Proof. This is an immediate corollary to Example 2.5.9 (v) and the observation that for $q \leq n, \pi_{R}=\tau \circ \pi_{H}$ where $\tau$ is the quotient map (2.6.1).

Lemma 4.3.9. Let $n=1$, and let $F: M \rightarrow M$ be $a \Gamma^{s}$ (possibly not contact) diffeomorphism, and let $s \geq 6(=2 n+4)$. Then $D_{R} \pi_{R} F^{*} \eta$ is in $\Gamma^{s-2}\left(R^{2}\right)$.

Proof. Recall the definition of the operator $D_{R}$ (see Section 2.6). For any $\alpha \in \mathcal{R}^{1} \subset \Omega^{1}(M), D_{R} \alpha=d(\widetilde{\alpha}+f \eta)$, where $\widetilde{\alpha} \in \Omega^{1}(M)$ is any form such that $\pi_{R}(\widetilde{\alpha})=\alpha$ and $f \in \mathcal{R}^{0}$ is the unique function such that $\eta \wedge d(\widetilde{\alpha}+f \eta)=0$. Let $\Lambda$ be the linear isomorphism

$$
\Lambda: \mathcal{R}^{0} \rightarrow \mathcal{R}^{3}=\Omega^{3}(M): h \mapsto h \eta \wedge d \eta
$$

Note that $\Lambda$ is defined by a smooth vector bundle isomorphism; it, therefore, extends to an isomorphism between the spaces $\Gamma^{s}(M)$ and $\Gamma^{s}\left(\Lambda^{3} M\right)$ for all $s$. Consider now the case $\alpha=\pi_{R} F^{*} \eta$. The condition defining $f$ is

$$
\eta \wedge\left(F^{*} d \eta+f d \eta\right)=0
$$

By Example 2.5.9(v), $\eta \wedge F^{*} d \eta$ is in $\Gamma^{s-1}\left(R^{3}\right)$, forcing $f$ to be in $\Gamma^{s-1}\left(R^{0}\right)$. Compute as follows

$$
D_{R}\left(\pi_{R} F^{*} \eta\right)=d F^{*} \eta+d(f \eta)=F^{*} d \eta+d f \wedge \eta+f d \eta .
$$

Since wedging with $\eta$ kills all terms in $d f \wedge \eta$ involving differentiation in directions transverse to the contact distribution, it follows that $d(f \eta)$ is in $\Gamma^{s-2}\left(\Lambda^{2} M\right)$. Thus, $D_{R} \alpha$ is in $\Gamma^{s-2}$, concluding the proof of the lemma. 
Remark 4.3.10. The result of Lemma 4.3.9 is somewhat surprising. Because $\pi_{R} F^{*} \eta$ is in $\Gamma^{s-1}$ and $D_{R}$ is an operator of contact order 2, one would expect $D_{R} \pi_{R} F^{*} \eta$ only to lie in $\Gamma^{s-3}$.

Proof of Proposition 4.3.7. By Proposition 4.1.2, the map $\chi^{s}: X \mapsto F_{X}$ is smooth; and for sufficiently small $X, F_{X}$ is a $\Gamma^{s}$-diffeomorphism. Recall that $\pi_{R} F^{*} \eta$ is in $\Gamma^{s-1}\left(R^{1}\right)$. The linear operators $d_{R}, \delta_{R}, D_{R}, D_{R}^{*}, H_{R}$ and $\pi_{R}$ are all bounded as maps as follows:

$$
\begin{aligned}
& d_{R}, \delta_{R}: \Gamma^{s} \rightarrow \Gamma^{s-1}, H_{R}: \Gamma^{s-1} \rightarrow \Gamma^{s}, \text { and } \pi_{R}: \Gamma^{s} \rightarrow \Gamma^{s}, \\
& G_{R}: \Gamma^{s}\left(R^{k}\right) \rightarrow \Gamma^{s+2}\left(R^{k}\right), \text { for } k<n, \\
& D_{R}, D_{R}^{*}: \Gamma^{s} \rightarrow \Gamma^{s-2}, \quad G_{R}: \Gamma^{s}\left(R^{k}\right) \rightarrow \Gamma^{s+4}\left(R^{k}\right), \text { for } k=n .
\end{aligned}
$$

and the individual terms have the following regularity properties:

$$
G_{R} \delta_{R}\left(\pi_{R} F_{X}^{*} \eta\right) \in \Gamma^{s}, \quad G_{R} \delta_{R} d_{R}\left(\pi_{R} F_{X}^{*} \eta\right) \in \Gamma^{s}, \quad H_{R}\left(\pi_{R} F_{X}^{*} \eta\right) \in C^{\infty},
$$

for $n>1$; and for $n=1$ :

$$
G_{R} \delta_{R}\left(\pi_{R} F_{X}^{*} \eta\right) \in \Gamma^{s}, \quad G_{R} D_{R}^{*} D_{R}\left(\pi_{R} F_{X}^{*} d \eta\right) \in \Gamma^{s}, \quad H_{R}\left(\pi_{R} F_{X}^{*} \eta\right) \in C^{\infty} .
$$

Thus, $\Phi$ is smooth and maps between the spaces as indicated.

The only non-linear terms in the map $\Phi$ arise from the presence of $F_{X}^{*} \eta$. The linearization of this term at the zero vector field is $\mathcal{L}_{X} \eta=d X^{0}+$ $\left.X_{H}\right\lrcorner d \eta$. When we substitute this into the map $\Phi$, we obtain easily the linearization and its inverse.

Let $\mathcal{U} \subset \Gamma^{2 n+4}(T M)$ be an open neighbourhood of the zero section such that $F_{X}$ is a $\Gamma^{s}$-diffeomorphism for all $X \in \mathcal{U}^{s}:=\mathcal{U} \cap \Gamma^{s}(T M), s \geq 2 n+4$. The next theorem shows that the subset $\mathcal{V}^{s} \subset \mathcal{U}^{s}$ on which $F_{X}$ is a contact diffeomorphism is a smooth submanifold which is smoothly parameterized by the space of $\Gamma^{s}$-contact vector fields near zero.

Theorem 4.3.11. For $\mathcal{U}$ sufficiently small, for all $s \geq 2 n+4$, the set

$$
\mathcal{V}^{s}=\left\{X \in \mathcal{U}^{s}: \Phi(X)=\left(g_{X}, 0,0\right)\right\}
$$

is a smooth submanifold of $\mathcal{U}^{s}:=\mathcal{U} \cap \Gamma_{\text {cont }}^{s}(T M)$, smoothly parameterized by the map

$$
\Psi: \Gamma_{\text {cont }}^{s}(T M) \cap \mathcal{U}^{s} \rightarrow \mathcal{V}^{s}: X \mapsto \Phi^{-1}\left(g_{X} \oplus 0\right) .
$$

Moreover, the map $\Psi$ is of the form

$$
\Psi(X)=X+B(X)(X, X)
$$

where $B:\left(\Gamma_{\text {cont }}^{s}(T M) \cap \mathcal{U}\right) \times \Gamma_{\text {cont }}^{s}(T M) \times \Gamma_{\text {cont }}^{s}(T M) \rightarrow \Gamma^{s}(T M)$ is smooth and bilinear in the last two factors. 
Proof. It suffices to prove the theorem for $s=2 n+4$. That $\mathcal{V}^{s} \subset \mathcal{U}^{s}$ is a smooth submanifold follows from Proposition 4.3.7 and the inverse function theorem in Banach spaces. To define $\Psi$, let $\pi$ denote the projection

$$
\pi(g, \gamma)=(g, 0)
$$

in the notation in the statement of Proposition 4.3.7, and let $\Psi=$

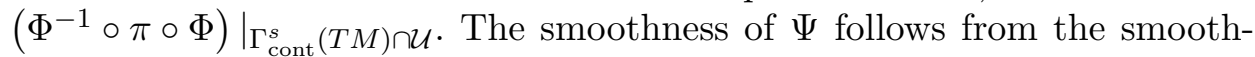
ness of $\Phi$. A simple calculation shows that $\left.d \Psi\right|_{0}(X)=X$, for all $X \in$ $\Gamma_{\text {cont }}^{s}(T M)$, which (together with the inverse function theorem) shows that $\Psi$ parameterizes $\mathcal{V}^{s}$. The form of the operator $B$ is given by Taylor's formula with integral remainder for smooth operators on Banach spaces:

$$
B(X)=\int_{0}^{1}(1-t) D^{2} \Psi(t X) d t .
$$

(See, e.g. [Ham, Theorem 3.5.6].)

Remark 4.3.13. In view of the isomorphism $\Gamma^{s+1}(M) \simeq \Gamma_{\text {cont }}^{s}(T M)$ given by (2.7.2), the map $g \mapsto \Psi\left(X_{g}\right)$ defines a smooth parameterization of $\mathcal{V}^{s}$ by $\Gamma^{s+1}$-functions in a neighbourhood of $0 \in \Gamma^{s+1}(M, \mathbb{R})$.

4.4. The smooth structure on the space of contact diffeomorphisms. The map $\Psi$ of Theorem 4.3.7 gives a parameterization of the subspace $\mathcal{V}^{s} \subset \mathcal{U}^{s}$. We now show that this parameterization in turn induces a smooth structure on the space $\mathcal{D}_{\text {cont }}^{s}(M)$ of all $\Gamma^{s}$-contact diffeomorphisms.

Theorem 4.4.1. Let $(M, \eta)$ be a compact contact manifold. For $s \geq 2 n+4$, the space of $\Gamma^{s}$ contact diffeomorphisms is a smooth Hilbert manifold.

Proof. We first show that the intersection of $\mathcal{D}_{\text {cont }}^{s}(M)$ with a neighbourhood of the identity is a smooth submanifold of $\mathcal{D}^{s}(M)$. To see this, let $\chi^{s}$ : $\mathcal{U}^{s} \rightarrow \mathcal{D}^{s}(M)$ be the diffeomorphism onto a neighbourhood of the identity given in Proposition 4.1.2. By Theorem 4.3.11, we can shrink $\mathcal{U}^{s}$ if necessary so that $\mathcal{V}^{s}$ is a smooth submanifold of $\mathcal{U}^{s}$. Now set $\mathcal{O}_{\text {Id }}^{s}=\chi^{s}\left(\mathcal{U}^{s}\right)$. Since $\chi^{s}: \mathcal{U}^{s} \rightarrow \mathcal{O}_{\text {Id }}^{s}$ is a diffeomorphism and

$$
\mathcal{D}_{\text {cont }}^{s}(M) \cap \mathcal{O}_{\text {Id }}^{s}=\chi^{s}\left(\mathcal{V}^{s}\right),
$$

it follows that $\mathcal{D}_{\text {cont }}^{s}(M) \cap \mathcal{O}_{\text {Id }}^{s}$ is a smooth submanifold of $\mathcal{D}^{s}(M)$.

Next consider the open set $\mathcal{O}_{F}^{s}=F\left(\mathcal{O}_{\text {Id }}^{s}\right) \subset \mathcal{D}^{s}(M)$, where $F$ is an arbitrary $C^{\infty}$ contact diffeomorphism. Noting that $G \in \mathcal{D}^{s}(M)$ is a contact diffeomorphism if and only if $F^{-1} \circ G$ is a contact diffeomorphism shows that the equality

$$
\mathcal{O}_{F}^{s} \cap \mathcal{D}_{\text {cont }}^{s}(M)=F\left(\mathcal{V}^{s}\right)
$$

holds. Finally, recall that composition on left with $F$ is a smooth diffeomorphism of $\mathcal{D}^{s}(M)$ (see Remark (4.1.3)) to conclude that $\mathcal{O}_{F}^{s} \cap \mathcal{D}_{\text {cont }}^{s}(M)$ is a smooth submanifold of $\mathcal{D}^{s}(M)$. 
It remains only to show that every element of $\mathcal{D}_{\text {cont }}^{s}(M)$ is contained in $\mathcal{O}_{F}^{s}$ for some smooth contact diffeomorphism $F$. To see this, choose any $G \in \mathcal{D}_{\text {cont }}^{s}(M)$ and let $F_{k}$ be a sequence of smooth contact diffeomorphisms converging to $G$. Because composition and inversion are continuous operations, $F_{k}^{-1} \circ G \rightarrow i d_{M}$ as $k \rightarrow \infty$. Therefore, $F_{k}^{-1} \circ G \in \mathcal{O}_{\text {Id }}^{s}$ for $k$ sufficiently large. Consequently, $G$ is contained in $\mathcal{O}_{F_{k}}^{s}=F_{k}\left(\mathcal{O}_{i d_{M}}^{s}\right)$ for $k$ sufficiently large.

Remark 4.4.2. We can construct a smooth atlas for $\mathcal{D}_{\text {cont }}^{s}(M)$ as follows. By Theorem 4.3.11 and Remark 4.3.13, there is an open neighbourhood $\mathcal{O}^{s+1}$ of $0 \in \Gamma^{s+1}(M, \mathbb{R})$ such that

$$
\chi_{\text {cont }}^{s+1}: \mathcal{O}^{s+1} \rightarrow \mathcal{D}_{\text {cont }}^{s}(M): g \mapsto \chi^{s} \circ \Psi^{s}\left(X_{g}\right)
$$

is a homeomorphism onto $\mathcal{O}_{\text {Id }}^{s} \cap \mathcal{D}_{\text {cont }}^{s}(M)$. Its inverse is a coordinate chart centred at the identity diffeomorphism. Composition with a smooth contact diffeomorphism $G$ then yields the map

$$
\chi_{\text {cont }, G}^{s+1}: \mathcal{O}^{s+1} \rightarrow \mathcal{D}_{\text {cont }}^{s}(M): g \mapsto G \circ \chi_{\text {cont }}^{s+1}(g),
$$

whose inverse is a coordinate chart centred at $G$. The argument in the last paragraph of the proof of Theorem 4.4.1 shows that the set of all such charts forms a smooth atlas for $\mathcal{D}_{\text {cont }}^{s}(M)$.

We next address the global topology of $T \mathcal{D}_{\text {cont }}^{s}(M)$. Because $\mathcal{D}_{\text {cont }}^{s}(M)$ is a closed submanifold of $\mathcal{D}^{s}(M)$, there is a smooth inclusion $T \mathcal{D}_{\text {cont }}^{s}(M) \subset$ $T \mathcal{D}^{s}(M)$. Using the fact that $\mathcal{D}^{s}(M)$ is an open subset of $\Gamma^{s}(M, M)$, and letting $\Gamma^{s}(M,|T M|)$ denote the space of $\Gamma^{s}$-maps from $M$ into the total space of $T M$ (i.e., forgetting the vector bundle structure on $T M$ ), one sees immediately that the tangent bundle of $\mathcal{D}^{s}(M)$ is the open subset

$$
\operatorname{TD}^{s}(M)=\left\{X \in \Gamma^{s}(M,|T M|): \pi \circ X \in \mathcal{D}^{s}(M)\right\}
$$

with bundle projection $T \mathcal{D}^{s}(M) \rightarrow \mathcal{D}^{s}(M): X \mapsto \pi \circ X$. A standard computation with Lie derivatives applied to a one-parameter family of contact diffeomorphisms then shows that a $\Gamma^{s}$-vector field $X: M \rightarrow|T M|$ is in $T \mathcal{D}_{\text {cont }}^{s}(M)$ if and only if $F=\pi \circ X$ is $\mathcal{D}_{\text {cont }}^{s}(M)$ and $X \circ F^{-1} \in \Gamma^{s}(T M)$ is a contact vector field. As the next proposition shows, $T \mathcal{D}_{\text {cont }}^{s}(M)$ is a trivial vector bundle:

Proposition 4.4.4. Let $X_{g}$ denote the contact vector field associated to the generating function $g$. The map

$$
\mathcal{D}_{\text {cont }}^{s}(M) \times \Gamma^{s}(M, \mathbb{R}) \longrightarrow T \mathcal{D}_{\text {cont }}^{s}(M):(g, F) \mapsto X_{g} \circ F,
$$

is a continuous vector bundle isomorphism.

Remark 4.4.5. Composition with a $\Gamma^{s}$-contact diffeomorphism is a continuous, but not differentiable, operation. Consequently, the trivialization in 
Proposition 4.4.4 is not smooth. We discuss the smoothness of composition in Section 4.5.

Proposition 4.4.4 is a corollary to a more general construction. Let $\pi: E \rightarrow M$ be a smooth vector bundle over $M$, and let $|E|$ denote the total space of $E$, viewed as a smooth manifold, forgetting its vector bundle structure. Recall that $\Gamma^{s}(M,|E|)$ denotes the space of $\Gamma^{s}$-maps from $M$ into $|E|$. Because $\pi$ is smooth, Corollary 2.4.5 applies to show that the map

$$
L_{\pi}^{s}: \Gamma^{s}(M,|E|) \rightarrow \Gamma^{s}(M, M): G \mapsto \pi \circ G .
$$

is smooth. Let $\Gamma_{\mathcal{D}_{\text {cont }}}^{s}(M, E)=\left(L_{\pi}^{s}\right)^{-1}\left(\mathcal{D}_{\text {cont }}^{s}(M)\right) \subset \Gamma^{s}(M,|E|)$ and let

$$
\pi_{\mathcal{D}}: \Gamma_{\mathcal{D}_{\text {cont }}}^{s}(M, E) \longrightarrow \mathcal{D}_{\text {cont }}^{s}(M)
$$

be the restriction of $L_{\pi}^{s}$ to $\Gamma_{\mathcal{D}_{\text {cont }}}^{s}(M, E)$. Note that the vector bundle structure on $E$ induces a vector space structure on the the fibres of $\pi_{\mathcal{D}}$, and we call $\pi_{\mathcal{D}}: \Gamma_{\mathcal{D}_{\text {cont }}}^{s}(M, E) \rightarrow \mathcal{D}_{\text {cont }}^{s}(M)$ the (vector) bundle of $\Gamma^{s}$-sections of $E$ over contact diffeomorphisms.

Lemma 4.4.7. Let $\pi: E \rightarrow M$ be a smooth vector bundle over $M$. Then for $s \geq 2 n+4$, the map $\Phi_{E}: \mathcal{D}_{\text {cont }}^{s}(M) \times \Gamma^{s}(E) \rightarrow \Gamma_{\mathcal{D}_{\text {cont }}}^{s}(M, E):(F, \sigma) \mapsto \sigma \circ F$ is a continuous, vector bundle isomorphism between $\Gamma_{\mathcal{D}_{\text {cont }}}^{s}(M, E)$ and the trivial vector bundle.

Proof. Consider first the special case where $E \rightarrow M$ is the trivial bundle $M \times \mathbb{R}^{r} \rightarrow M$. The diffeomorphism $\Gamma^{s}\left(M,\left|M \times \mathbb{R}^{r}\right|\right) \simeq \Gamma^{s}(M, M) \times \Gamma^{s}\left(M, \mathbb{R}^{r}\right)$ restricts to a diffeomorphism

$$
\Gamma_{\mathcal{D}_{\text {cont }}}^{s}\left(M, M \times \mathbb{R}^{r}\right) \simeq \mathcal{D}_{\text {cont }}^{s}(M) \times \Gamma^{s}\left(M, \mathbb{R}^{r}\right)
$$

with respect to which $\Phi_{M \times \mathbb{R}^{r}}$ assumes the form

$\Phi_{M \times \mathbb{R}^{r}}: \mathcal{D}_{\text {cont }}^{s}(M) \times \Gamma^{s}\left(M, \mathbb{R}^{r}\right) \rightarrow \Gamma_{\mathcal{D}_{\text {cont }}}^{s}\left(M, M \times \mathbb{R}^{r}\right):(F, \sigma) \mapsto(F, \sigma \circ F)$, with inverse

$\Phi_{M \times \mathbb{R}^{r}}^{-1}: \Gamma_{\mathcal{D}_{\text {cont }}}^{s}\left(M, M \times \mathbb{R}^{r}\right) \rightarrow \mathcal{D}_{\text {cont }}^{s}(M) \times \Gamma^{s}\left(M, \mathbb{R}^{r}\right):(F, \sigma) \mapsto\left(F, \sigma \circ F^{-1}\right)$.

Continuity of $\Phi_{M \times \mathbb{R}^{r}}^{-1}$ follows from continuity of composition with $F$ (see Corollary 3.1.4); and continuity of $\Phi_{M \times \mathbb{R}^{r}}^{-1}$ follows from continuity of inversion (see Theorem 3.2.5).

Now consider the general case. By construction, $\Phi_{E}$ is bijective, preserves basepoint, and is linear on each fibre. To see that $\Phi_{E}$ is continuous, note that since the map $E \rightarrow M \times E: e \mapsto(\pi(e), e)$ is smooth, so is the induced map

$$
\iota: \Gamma^{s}(E) \hookrightarrow \Gamma^{s}(M \times E)
$$

defined by the formula $\iota(\sigma): x \mapsto(x, \sigma(x))$. This observation, together with Corollary 2.4.5 implies continuity of $\Phi_{E}$. It remains only to show that $\Phi_{E}^{-1}$ is continuous. Let $j: E \rightarrow M \times \mathbb{R}^{r}$ be a smooth vector bundle inclusion into a trivial bundle, and let $s: M \times \mathbb{R}^{r} \rightarrow E$ be a smooth vector bundle 
map with $s \circ j=i d_{E}$. Continuity of $\Phi_{E}^{-1}$ is proved by expressing $\Phi_{E}^{-1}$ as the following composition of continuous maps:

$$
\begin{aligned}
\Gamma_{\mathcal{D}_{\text {cont }}}^{s}(M, E) & \stackrel{L_{j}^{s}}{\longrightarrow} \Gamma_{\mathcal{D}_{\text {cont }}}^{s}\left(M, M \times \mathbb{R}^{r}\right) \simeq \mathcal{D}_{\text {cont }}^{s}(M) \times \Gamma^{s}\left(M, \mathbb{R}^{r}\right) \\
& \stackrel{\Phi_{M \times \mathbb{R}^{r}}^{-1}}{\longrightarrow} \mathcal{D}_{\text {cont }}^{s}(M) \times \Gamma^{s}\left(M, \mathbb{R}^{r}\right) \\
& \stackrel{i d_{\mathcal{D}_{\text {cont }}^{s}(M)} \times L_{s}^{s}}{\longrightarrow} \mathcal{D}_{\text {cont }}^{s}(M) \times \Gamma^{s}(M, E),
\end{aligned}
$$

concluding the proof of the lemma.

We close this section with a formula for the derivative of $\chi_{\text {cont }}^{s}$, which we need in Section 4.5. For $g \in \mathcal{O}^{s+1}$, we denote by $T_{F_{g}} \mathcal{D}_{\text {cont }}^{s}(M)$ the tangent space to $\mathcal{D}_{\text {cont }}^{s}(M)$ at the contact diffeomorphism $F_{g}$, and for $h \in \Gamma^{s+1}(M, \mathbb{R})$, we set

$$
Y_{(g, h)}=D \chi_{\operatorname{cont} g}^{s+1}(h): M \rightarrow T M,
$$

where $Y_{(g, h)} \in T_{F_{g}} \mathcal{D}_{\text {cont }}^{s}(M)$; and we set $X_{(g, h)}=Y_{(g, h)} \circ F_{g}^{-1} \in \Gamma_{\text {cont }}^{s}(T M)$.

Lemma 4.4.8. For $s \geq 2 n+4$, the map

$$
\mathcal{O}^{s+1} \times \Gamma^{s+2}(M, \mathbb{R}) \rightarrow \Gamma_{\text {cont }}^{s}(T M):(g, h) \mapsto X_{(g, h)}
$$

is continuous. Moreover, for every $g \in \mathcal{O}^{s+1}$ and $\epsilon>0$, there is a $\delta>0$ such that

$$
\left\|X_{\left(g_{1}, h\right)}-X_{(g, h)}\right\|_{s}<\epsilon\|h\|_{s+1}
$$

for all $g_{1} \in \mathcal{O}^{s+1}$ such that $\left\|g_{1}-g\right\|_{s+1}<\delta$ and all $h \in \Gamma^{s+2}(M, \mathbb{R})$.

Proof. Continuity of the map is clear. The estimate is the restatement of the fact that the derivative $D \chi_{\text {cont }, g}^{s}$ depends continuously on $g$.

4.5. Differentiability of composition. We showed in Section 3 that composition

$$
\mu: \mathcal{D}_{\text {cont }}^{s}(M) \times \Gamma^{k}(M, \mathbb{R}) \rightarrow \Gamma^{k}(M, \mathbb{R}):(F, u) \mapsto u \circ F
$$

is a continuous operation for $2 n+4 \leq k \leq s$, but composition is not $C^{1}$, as the following counterexample shows. Choose $u \in \Gamma^{k}(M, \mathbb{R})$ with $T(u) \notin$ $\Gamma^{k}(M \mathbb{R})$, where $T$ is the Reeb vector field on $M$. Then the one-parameter family $F_{t}$ of contact diffeomorphisms given by the flow of the Reeb vector field $T$ is a smooth curve in $\mathcal{D}_{\text {cont }}^{s}(M)$; and differentiability of $\mu$ would imply that the limit

$$
\lim _{t \rightarrow 0} \frac{\mu\left(u, F_{t}\right)-\mu\left(u, F_{0}\right)}{t}=T(u)
$$

would be an element of $\Gamma^{k}(M, \mathbb{R})$. But this contradicts our choice of $u$. The next theorem shows that we can recover smoothness by strengthening the regularity assumption on $u$. 
Theorem 4.5.1. Let $M$ be a compact contact manifold of dimension $2 n+1$ and let $N$ be a smooth manifold. Then the map

$$
\mu: \mathcal{D}_{\text {cont }}^{s}(M) \times \Gamma^{k+2}(M, N) \rightarrow \Gamma^{k}(M, N):(F, G) \mapsto G \circ F
$$

is continuously differentiable for $2 n+4 \leq k \leq s$. In case $N=\mathbb{R}^{m}$, $\mu$ is continuously differentiable for $0 \leq k \leq s, 2 n+4 \leq s$.

Proof. Assume that the theorem holds in the special case where $N=\mathbb{R}^{m}$, with $m$ arbitrary. Let $\iota: N \hookrightarrow \mathbb{R}^{m}$ be a closed embedding, and let $U \subset \mathbb{R}^{m}$ be a tubular neighbourhood of $N$, with projection map $\pi: U \rightarrow N$. By Corollary 2.4.5, the maps $\iota$ and $\pi$ induce smooth maps

$$
\widetilde{\iota}: \Gamma^{k}(M, N) \rightarrow \Gamma^{k}(M, U) \quad \text { and } \quad \widetilde{\pi}: \Gamma^{k}(M, U) \rightarrow \Gamma^{k}(M, N)
$$

for all $k \geq 2 n+4$. Because $\Gamma^{k}(M, U)$ is an open subset of $\Gamma^{k}\left(M, \mathbb{R}^{m}\right)$ by assumption, we know that

$$
\mu: \mathcal{D}_{\text {cont }}^{s}(M) \times \Gamma^{k+2}(M, U) \rightarrow \Gamma^{k}(M, U):(F, u) \mapsto u \circ F
$$

is a $C^{1}$ map. It follows that the composition

$$
\begin{aligned}
\mathcal{D}_{\text {cont }}^{s}(M) \times \Gamma^{k+2}(M, N) \stackrel{i d \times \widetilde{\iota}}{\longrightarrow} \mathcal{D}_{\text {cont }}^{s}(M) \times \Gamma^{k+2}(M, U) \stackrel{\mu}{\longrightarrow} \Gamma^{k}(M, U) \\
\stackrel{\tilde{\pi}}{\longrightarrow} \Gamma^{k}(M, N)
\end{aligned}
$$

is a $C^{1}$ map.

It remains to prove the theorem in the case $N=\mathbb{R}^{m}$. Because $\Gamma^{k}\left(M, \mathbb{R}^{m}\right)$ is the $m$ th fold product of $\Gamma^{k}(M, \mathbb{R})$, we need only to prove it for $m=1$; and by Remark 4.4.2, it suffices to restrict to an open neighbourhood of the identity in $\mathcal{D}_{\text {cont }}^{s}(M)$. Now for $\mathcal{O}^{s+1} \subset \Gamma^{s+1}(M)$ a sufficiently small neighbourhood of 0 , the map

$$
\chi_{\text {cont }}^{s+1}: \mathcal{O}^{s+1} \longrightarrow \mathcal{D}_{\text {cont }}^{s}(M): g \mapsto F_{g}
$$

is a smooth parameterization of a neighbourhood of the identity contact diffeomorphism. (Here and in the following we set $F_{g}=F_{\Psi\left(X_{g}\right)}$, where $X_{g}$ denotes the contact vector field with generating function $g$.) With this notation, the proof reduces to proving that the map

$$
\mu: \Gamma^{k+2}(M, \mathbb{R}) \times \mathcal{O}^{s+1} \longrightarrow \Gamma^{k}(M, \mathbb{R}):(u, g) \mapsto u \circ F_{g}
$$

is $C^{1}$. The next proposition completes the proof.

Proposition 4.5.2. For $s \geq 2 n+4$ and $s \geq k \geq 0$ and for $\mathcal{O}^{s+1} \subset$ $\Gamma^{s+1}(M, \mathbb{R})$ a sufficiently small neighbourhood of 0 , the map

$$
\mu: \Gamma^{k+2}(M, \mathbb{R}) \times \mathcal{O}^{s+1} \longrightarrow \Gamma^{k}(M, \mathbb{R}):(u, g) \mapsto u \circ F_{g} .
$$

is $C^{1}$ with derivative at $(u, g)$ given by the formula

$$
\left.D \mu_{(u, g)}:(v, h) \mapsto v \circ F_{g}+\left(X_{(g, h)}\right\rfloor d u\right) \circ F_{g},
$$

for $(v, h) \in \Gamma^{k+2}(M, \mathbb{R}) \times \Gamma^{s+1}(M, \mathbb{R})$. 
Proof. Because $\mu$ is a map between Banach spaces, to show that it is $C^{1}$, we need only to show that the two partial derivatives of $\mu$ with respect to the first and second variables

$$
\begin{aligned}
& D_{1} \mu: \Gamma^{k+2}(M, \mathbb{R}) \times \mathcal{O}^{s+1} \rightarrow L\left(\Gamma^{k+2}(M, \mathbb{R}), \Gamma^{k}(M, \mathbb{R})\right), \\
& D_{2} \mu: \Gamma^{k+2}(M, \mathbb{R}) \times \mathcal{O}^{s+1} \rightarrow L\left(\Gamma^{s+1}(M, \mathbb{R}), \Gamma^{k}(M, \mathbb{R})\right)
\end{aligned}
$$

exist and are continuous ${ }^{3}$. We shall obtain formulas for $D_{1} \mu$ and $D_{2} \mu$. The formula for $D \mu_{(u, g)}(v, h)$ then follows immediately from the well-known identity

$$
D \mu_{(u, g)}(v, h)=D_{1} \mu_{(u, g)}(v)+D_{2} \mu_{(u, g)}(h) .
$$

To see that $D_{1} \mu$ exists, note that by Corollary 3.1.4, $\mu$ is continuous. The map $\mu$ is linear in the first variable and, therefore, differentiable with respect to the first variable, with derivative given by $D_{1} \mu_{(u, g)}(v)=v \circ F_{g}$. Continuity of $D_{1} \mu$ is proved in Lemma 4.5.4 below.

We next claim that

$$
\left.D_{2} \mu_{(u, g)}(h)=\left(X_{(g, h)}\right\lrcorner d u\right) \circ F_{g} .
$$

To prove the claim, first note that because $s \geq 2 n+4$, the functions $g, h$, $u$, as well as the map $F_{g}$, are of class at least $C^{1}$. Moreover, because $\chi_{\text {cont }}^{s+1}$ is smooth, the family $t \mapsto F_{g+t h}$ of contact diffeomorphisms is a smooth family. Consequently, we can compute pointwise at $x \in M$, employing the chain rule as follows:

$$
\begin{aligned}
D_{2} \mu_{(u, g)}(h)(x) & =\lim _{t \rightarrow 0} \frac{u\left(F_{g+t h}(x)\right)-u\left(F_{g}(x)\right)}{t}=\left.\frac{d u\left(F_{g+t h}(x)\right)}{d t}\right|_{t=0} \\
& \left.=d u\left(D \chi_{\text {cont }, g}^{s+1}(h)(x)\right)=Y_{(g, h)}(x)\right\lrcorner d u_{F_{g}(x)} \\
& \left.=\left(X_{(g, h)}\right\lrcorner d u\right) \circ F_{g}(x) .
\end{aligned}
$$

To prove that $\mu$ is differentiable with respect to the second variable, we need to verify the formula

$$
\lim _{h \rightarrow 0} \frac{\left.\| u \circ F_{g+h}-u \circ F_{g}-\left(X_{(g, h)}\right\rfloor d u\right) \circ F_{g} \|_{k}}{\|h\|_{s+1}}=0
$$

and we need to prove continuity of $D_{2} \mu$. We do this in Lemma 4.5.10.

Lemma 4.5.4. $D_{1} \mu: \Gamma^{k+2}(M, \mathbb{R}) \times \mathcal{O}^{s+1} \rightarrow L\left(\Gamma^{k+2}(M, \mathbb{R}), \Gamma^{k}(M, \mathbb{R})\right)$ is continuous for $s \geq 2 n+4$ and all $k \geq 0$.

Proof. By definition of continuity, we must show that for any $g_{0} \in \mathcal{O}^{s+1}$ and any $\epsilon>0$, there is a $\delta>0$, such that the condition

$$
\left\|v \circ F_{g}-v \circ F_{g_{0}}\right\|_{k}<\epsilon\|v\|_{k+2}
$$

\footnotetext{
${ }^{3}$ We use the notation $L\left(\mathcal{H}_{1}, \mathcal{H}_{2}\right)$ to denote the Banach space of bounded linear maps between Hilbert spaces $\mathcal{H}_{1}$ and $\mathcal{H}_{2}$.
} 
is satisfied for all $v \in \Gamma^{k}(M, \mathbb{R})$ and all $g \in \mathcal{O}^{s+1}$ with $\left\|g-g_{0}\right\|_{s+1}<\delta$. We need only to prove the estimate for $v$ a smooth test function.

Set $h=g-g_{0}$. Because the map $g \mapsto F_{g}$ is smooth, and $v$ is smooth, for fixed $x \in M$, the function $\gamma_{x}: t \mapsto v\left(F_{g_{0}+t h}(x)\right)$ is a $C^{1}$ function of $t$. Consequently, we can compute as follows using the chain rule:

$$
\begin{aligned}
v \circ F_{g}(x)-v \circ F_{g_{0}}(x) & =\int_{0}^{1} \frac{d}{d t} v\left(F_{g_{0}+t h}(x)\right) d t \\
& \left.=\int_{0}^{1}\left(X_{\left(g_{0}+t h, h\right)}\right\rfloor d v\right) \circ F_{g_{0}+t h}(x) d t .
\end{aligned}
$$

Viewing $\left.t \mapsto\left(X_{\left(g_{0}+t h, h\right)}\right\rfloor d v\right) \circ F_{g_{0}+t h}$ as a continuous curve in the Hilbert space $\Gamma^{k}(M, \mathbb{R})$ yields the inequality

$$
\left.\left\|v \circ F_{g}(x)-v \circ F_{g_{0}}(x)\right\|_{k} \leq \int_{0}^{1} \|\left(X_{\left(g_{0}+t h, h\right)}\right\lrcorner d v\right) \circ F_{g_{0}+t h} \|_{k} d t .
$$

Hence, to prove the lemma it suffices to show that, for $\delta>0$ sufficiently small, the estimate

$$
\left.\|\left(X_{g, h}\right\lrcorner d v\right) \circ F_{g}\left\|_{k}<\epsilon\right\| v \|_{k+2}
$$

holds for all $g \in \mathcal{O}^{s+1}$ and $h \in \Gamma^{s+1}(M, \mathbb{R})$ with $\left\|g-g_{0}\right\|_{s+1}<\delta$ and $\|h\|_{s+1}<\delta$. To obtain (4.5.5), first observe that since interior evaluation

$$
\left.\Gamma^{s}(T M) \times \Gamma^{k}\left(T^{*} M\right) \rightarrow \Gamma^{k}(M, \mathbb{R}):(X, \beta) \mapsto X\right\lrcorner \beta
$$

is a smooth bilinear map (see Proposition 2.4.2), the estimate

$$
\| X\lrcorner \beta\left\|_{k} \prec\right\| X\left\|_{s}\right\| \beta \|_{k}
$$

holds. Note also that by continuity of composition (see Corollary 3.1.4) and linearity in $v$,

$$
\left\|v \circ F_{g_{0}}\right\|_{k} \prec\|v\|_{k}
$$

for all $v$. Continuity also shows that we can choose $\delta>0$ small, so that

$$
\left\|v \circ F_{g}-v \circ F_{g_{0}}\right\|_{k}<1
$$

holds, provided $\|v\|_{k}<\delta$ and $\left\|g-g_{0}\right\|_{s+1}<\delta$. By linearity in $v$, setting $C_{2}=1 / \delta$, we get the estimate

$$
\left\|v \circ F_{g}-v \circ F_{g_{0}}\right\|_{k}<C_{2}\|v\|_{k}
$$

provided $\left\|g-g_{0}\right\|_{s+1}<\delta$. Finally, note that because $\chi_{\text {cont }}^{s+1}$ is smooth, its derivative $D \chi_{\text {cont }}^{s+1}$ is continuous in the operator norm. We can therefore choose $\delta>0$ so small that

$$
\left\|X_{(g, h)}-X_{\left(g_{0}, h\right)}\right\|_{s}<\epsilon\|h\|_{s+1}
$$


for all $h$, provided $\left\|g-g_{0}\right\|_{s+1}<\delta$. Choosing $\delta>0$, so that all of the above estimates hold and choosing $g$ so that $\left\|g-g_{0}\right\|_{s+1}<\delta$, we can then estimate as follows:

$$
\begin{aligned}
\| & \left.\left(X_{(g, h)}\right\lrcorner d v\right) \circ F_{g} \|_{k} \\
& \left.\left.\left.\leq \|\left(X_{(g, h)}\right\lrcorner d v\right) \circ F_{g_{0}}\left\|_{k}+\right\|\left(X_{(g, h)}\right\lrcorner d v\right) \circ F_{g}-\left(X_{(g, h)}\right\lrcorner d v\right) \circ F_{g_{0}} \|_{k} \\
& \left.\left.\left.\left.\prec \|\left(X_{(g, h)}\right\lrcorner d v\right) \circ F_{g_{0}}\left\|_{k}+\right\| X_{(g, h)}\right\rfloor d v\left\|_{k} \prec\right\| X_{(g, h)}\right\lrcorner d v\left\|_{k}+\right\| X_{(g, h)}\right\lrcorner d v \|_{k} \\
& \left.\prec \| X_{(g, h)}\right\lrcorner d v\left\|_{k} \prec\right\| X_{(g, h)}\left\|_{s} \cdot\right\| v \|_{k+2} \\
& \left.\prec\left(\|\left(X_{\left(g_{0}, h\right)}\right\lrcorner d v\right)\left\|_{s}+\epsilon\right\| h \|_{s+1}\right) \cdot\|v\|_{k+2} \\
& \prec\|h\|_{s+1} \cdot\|v\|_{k+2} .
\end{aligned}
$$

The estimate (4.5.5) follows by decreasing $\delta$, if necessary, and requiring $\|h\|_{s+1}<\delta$.

Lemma 4.5.10. For $2 n+4 \leq s, k \leq s$, and $\mathcal{O}^{s+1}$ as in Lemma 4.5.4, the derivative $D_{2} \mu: \Gamma^{k+2}(M, \mathbb{R}) \times \mathcal{O}^{s+1} \rightarrow L\left(\Gamma^{s+1}(M, \mathbb{R}), \Gamma^{k}(M, \mathbb{R})\right)$ exists, is continuous, and given by the formula

$$
\left.D_{2} \mu_{(u, g)}(h)=Y_{(g, h)}\right\lrcorner\left(d u \circ F_{g}\right),
$$

for $g \in \mathcal{O}^{s+1}, u \in \Gamma^{k}(M, \mathbb{R})$, and $h \in \Gamma^{s+1}(M, \mathbb{R})$.

Proof. To prove that $D_{2} \mu$ exists, choose $\left(u, g_{0}\right) \in \Gamma^{k+2}(M, \mathbb{R}) \times \mathcal{O}^{s+1}$. We need to show that

$$
\lim _{h \rightarrow 0} \frac{\left.\| u \circ F_{g_{0}+h}-u \circ F_{g_{0}}-\left(X_{\left(g_{0}, h\right)}\right\rfloor d u\right) \circ F_{g_{0}} \|_{k}}{\|h\|_{s+1}}=0 .
$$

Choose $\epsilon>0$. We need to find $\delta>0$ such that

$$
\left.\| u \circ F_{g_{0}+h}-u \circ F_{g_{0}}-\left(X_{\left(g_{0}, h\right)}\right\lrcorner d u\right) \circ F_{g_{0}}\left\|_{k}<\epsilon\right\| h \|_{s+1} \text { for }\|h\|_{s+1}<\delta .
$$

To this end, compute as follows for $g \in \mathcal{O}^{s+1}$ near $g_{0}$, setting $h=g-g_{0}$ :

$$
\begin{aligned}
\| u & \left.\circ F_{g_{0}+h}-u \circ F_{g_{0}}-\left(X_{\left(g_{0}, h\right)}\right\rfloor d u\right) \circ F_{g} \|_{k} \\
& \left.=\| \int_{0}^{1}\left\{\frac{d\left(u \circ F_{g_{0}+t h}\right)}{d t}-\left(X_{\left(g_{0}, h\right)}\right\rfloor d u\right) \circ F_{g_{0}}\right\} d t \|_{k} \\
& \left.\leq \int_{0}^{1} \|\left\{\frac{d\left(u \circ F_{g_{0}+t h}\right)}{d t}-\left(X_{\left(g_{0}, h\right)}\right\rfloor d u\right) \circ F_{g_{0}}\right\} \|_{k} d t \\
& \left.\left.=\int_{0}^{1} \|\left(X_{\left(g_{0}+t h, h\right)}\right\rfloor d u\right) \circ F_{g_{0}+t h}-\left(X_{\left(g_{0}, h\right)}\right\lrcorner d u\right) \circ F_{g_{0}} \|_{k} d t .
\end{aligned}
$$

Consequently, to prove (4.5.12), it suffices to find $\delta>0$ so that the inequality

$$
\left.\left.\|\left(X_{(g, h)}\right\lrcorner d u\right) \circ F_{g}-\left(X_{\left(g_{0}, h\right)}\right\lrcorner d u\right) \circ F_{g_{0}}\left\|_{k}<\epsilon\right\| h \|_{s+1}
$$

holds for all $\left\|g-g_{0}\right\|_{s+1}<\delta$. But by Proposition 4.5.2, we can choose $\delta>0$ so that 
$\left\|v \circ F_{g}-v \circ F_{g_{0}}\right\|_{k}<\epsilon\|v\|_{k+2}$ for all $v \in \Gamma^{k}(M, \mathbb{R})$; and with this choice of $\delta$, we can estimate as follows:

$$
\begin{aligned}
& \left.\left.\|\left(X_{(g, h)}\right\lrcorner d u\right) \circ F_{g}-\left(X_{\left(g_{0}, h\right)}\right\lrcorner d u\right) \circ F_{g_{0}} \|_{k} \\
& \left.\left.\left.\quad \leq \mid\left(X_{(g, h)}\right\lrcorner d u\right) \circ F_{g}-\left(X_{g, h}\right\lrcorner d u\right) \circ F_{g_{0}}\left\|_{k}+\right\|\left(X_{(g, h)}\right\lrcorner d u\right) \circ F_{g_{0}} \\
& \left.\quad-\left(X_{\left(g_{0}, h\right)}\right\lrcorner d u\right) \circ F_{g_{0}} \|_{k} \\
& \left.\left.\quad \prec \epsilon \| X_{(g, h)}\right\lrcorner d u\left\|_{k}+\right\|\left(X_{(g, h)}-X_{\left(g_{0}, h\right)}\right)\right\lrcorner d u \|_{k} \\
& \left.\quad \prec \epsilon \| X_{(g, h)}\right\lrcorner d u\left\|_{k}+\right\| X_{(g, h)}-X_{\left(g_{0}, h\right)}\left\|_{s} \cdot\right\| d u \|_{k} .
\end{aligned}
$$

Finally, using smoothness of $\chi_{\text {cont }}^{s+1}$ as we did in equation (4.5.9) above we can bound the last term as follows, for $\delta$ sufficiently small:

$$
\left.\prec \epsilon \| X_{(g, h)}\right\rfloor d u\left\|_{k}+\left(\epsilon\|h\|_{s+1}\right)\right\| d u\left\|_{k} \prec \epsilon\right\| h \|_{s+1} .
$$

This concludes the proof of (4.5.12). Continuity of $D_{2} \mu$ follows from the following estimate:

$$
\begin{aligned}
& \left\|D_{2} \mu_{(g, u)}(h)-D_{2} \mu_{\left(g_{0}, u_{0}\right)}(h)\right\|_{k} \\
& \left.\left.=\|\left(X_{(g, h)}\right\lrcorner d u\right) \circ F_{g}-\left(X_{\left(g_{0}, h\right)}\right\lrcorner d u_{0}\right) \circ F_{g_{0}} \|_{k} \\
& \prec \\
& \left.\left.\left.\quad \|\left(X_{(g, h)}\right\lrcorner d u\right) \circ F_{g}-\left(X_{\left(g_{0}, h\right)}\right\lrcorner d u\right) \circ F_{g_{0}}\left\|_{k}+\right\|\left(X_{\left(g_{0}, h\right)}\right\lrcorner d u\right) \circ F_{g_{0}} \\
& \left.\quad-\left(X_{\left(g_{0}, h\right)}\right\rfloor d u_{0}\right) \circ F_{g_{0}} \|_{k} \\
& \quad \prec \epsilon\|d u\|_{k}\|h\|_{s+1}+C\left\|d\left(u-u_{0}\right)\right\|\|h\|_{s+1} \prec \epsilon\|h\|_{s+1},
\end{aligned}
$$

which holds for all $(g, u)$ with $\left\|g-g_{0}\right\|_{s+1}<\delta$, $\left\|u-u_{0}\right\|_{k+1}<\delta$.

4.6. Some a priori estimates. Theorems 4.3 .11 and 4.4 .1 state that the non-linear space of $\Gamma^{s}$ contact diffeomorphisms is a Hilbert manifold modelled on the linear space of $\Gamma^{s}$ contact vector fields. In typical applications, one would like to study the action of the space of contact diffeomorphisms on some set of structures by comparing it with the linearized action of contact vector fields. For this strategy to work, it is necessary to show that the error incurred in the linearization is quadratically small in an appropriate sense. This is the content of the next proposition, which gives a priori estimates for the quadratic error $\Psi(X)-X=B(X)(X, X)$. We require this result in $[\mathbf{B D 3}]$ to obtain normal forms for CR structures on compact three-dimensional contact manifolds.

Proposition 4.6.1. For $X \in \Gamma_{\text {cont }}^{s}(T M) \cap \mathcal{U}^{s}$,

$$
\|\Psi(X)-X\|_{s} \prec\|X\|_{s}\|X\|_{s-1} .
$$

Moreover, for all $X_{1}, X_{2} \in \Gamma_{\text {cont }}^{s}(T M) \cap \mathcal{U}^{s}$,

(ii)

$$
\begin{aligned}
\left\|\left(\Psi\left(X_{2}\right)-X_{2}\right)-\left(\Psi\left(X_{1}\right)-X_{1}\right)\right\|_{s} \prec & \left\|X_{2}-X_{1}\right\|_{s-1}\left(\left\|X_{2}\right\|_{s}+\left\|X_{1}\right\|_{s}\right) \\
& +\left\|X_{2}-X_{1}\right\|_{s}\left(\left\|X_{2}\right\|_{s-1}+\left\|X_{1}\right\|_{s-1}\right) .
\end{aligned}
$$


Our proof relies on the next two lemmas. The first is a corollary to Lemma 4.2.7 and compactness of $M$. (See Remark 4.2.8 for the definition of $Q$.)

Lemma 4.6.2. For $c>0$ sufficiently small, the following estimates hold for $\psi$ a fixed smooth $q$ form. For all $X \in \Gamma_{\text {cont }}^{s+2}(T M), s \geq 2 n+4$, such that $|X|<c$ :

$$
\begin{aligned}
\left\|F_{X}^{*} \psi\right\|_{s} & \prec\|\psi\|_{s}+\left\|\mathcal{L}_{X} \psi\right\|_{s}+\|X\|_{s}\|X\|_{s+2} \\
\left\|\left(F_{X}^{*} \psi\right) \wedge \eta\right\|_{s} & \prec\|\wedge \eta\|_{s}+\left\|\mathcal{L}_{X} \psi \wedge \eta\right\|_{s}+\|X\|_{s}\|X\|_{s+1}
\end{aligned}
$$

and, for $q \leq n$,

$$
\left\|\pi_{R}\left(F_{X}^{*} \psi\right)\right\|_{s} \prec\left\|\pi_{R} \psi\right\|_{s}+\left\|\pi_{R}\left(\mathcal{L}_{X} \psi\right)\right\|_{s}+\|X\|_{s}\|X\|_{s+1} .
$$

Moreover, for $q \leq n$,

$$
\begin{aligned}
\left\|\pi_{R}\left(Q_{\psi}\left(X_{1}\right)-Q_{\psi}\left(X_{2}\right)\right)\right\|_{s} \prec & \left\|X_{1}-X_{2}\right\|_{s}\left(\left\|X_{1}\right\|_{s+1}+\left\|X_{2}\right\|_{s+1}\right) \\
& +\left\|X_{1}-X_{2}\right\|_{s+1}\left(\left\|X_{1}\right\|_{s}+\left\|X_{2}\right\|_{s}\right)
\end{aligned}
$$

holds for any two vector fields $X_{i}, i=1,2$ such that $\left|X_{i}\right|<c$.

Proof. Choose an adapted atlas and a constant $c>0$ as in the discussion above Lemma 4.2.7. The inequalities (i) and (ii) follow from the definition of $Q_{\psi}$. To prove (iii), note that by Lemma $4.2 .7, \pi_{R} \circ Q_{\psi}$ is a smooth differential operator of contact order 1 ; the inequality then follows. The inequality (iv) follows from the smooth dependence of $Q_{\psi}(X)$ on $X$.

Lemma 4.6.3. Choose $c>0$ as in the previous lemma. The following estimates are satisfied for any $\Gamma^{s+2}$ vector fields $X, X_{i}, i=1,2$ with $|X|<c$, $\left|X_{i}\right|<c, s \geq 2 n+4$. If $n=1$ then

$$
\left\|D_{R} \pi_{R}\left(F_{X}^{*} \eta-\eta-\mathcal{L}_{X} \eta\right)\right\|_{s-2}=\left\|D_{R} \pi_{R}\left(Q_{\eta}(X)\right)\right\|_{s-2} \prec\|X\|_{s}\|X\|_{s-1}
$$

and

$$
\begin{aligned}
\left\|D_{R} \pi_{R}\left(Q_{\eta}\left(X_{1}\right)-Q_{\eta}\left(X_{2}\right)\right)\right\|_{s-2} \prec & \left(\left\|X_{1}\right\|_{s}+\left\|X_{2}\right\|_{s}\right) \cdot\left\|X_{1}-X_{2}\right\|_{s-1} \\
& +\left(\left\|X_{1}\right\|_{s-1}+\left\|X_{2}\right\|_{s-1}\right) \cdot\left\|X_{1}-X_{2}\right\|_{s} .
\end{aligned}
$$

If $n>1$ then

$$
\left\|d_{R} \pi_{R}\left(F_{X}^{*} \eta-\eta-\mathcal{L}_{X} \eta\right)\right\|_{s-1}=\left\|d_{R} \pi_{R}\left(Q_{\eta}(X)\right)\right\|_{s-1} \prec\|X\|_{s}\|X\|_{s-1}
$$

and

$$
\begin{aligned}
\left\|d_{R} \pi_{R}\left(Q_{\eta}\left(X_{1}\right)-Q_{\eta}\left(X_{2}\right)\right)\right\|_{s-1, l o c} \prec & \left(\left\|X_{1}\right\|_{s}+\left\|X_{2}\right\|_{s}\right) \cdot\left\|X_{1}-X_{2}\right\|_{s-1} \\
& +\left(\left\|X_{1}\right\|_{s-1}+\left\|X_{2}\right\|_{s-1}\right) \cdot\left\|X_{1}-X_{2}\right\|_{s} .
\end{aligned}
$$


Proof. Let $\phi=F_{X}^{*} \eta-\eta-\mathcal{L}_{X} \eta \in \Gamma^{s}\left(\Lambda^{1}(M)\right.$. By compactness of $M$, it suffices to obtain local estimates on a coordinate patch $W \Subset U$ chosen as in Lemma 4.2.7. In the notation of Lemma 4.2.7, the one form $\phi$ can be written

$$
\begin{aligned}
\phi & =Q_{i j}^{1}(X) X^{i} X^{j}+Q_{i j}^{2}(X) X^{i} d X^{j} \\
& =Q_{i j, k}(x, X(x)) X^{i}(x) X^{j}(x) d x^{k}+Q_{i j}(x, X(x)) X^{i}(x) d X^{j}(x),
\end{aligned}
$$

where $Q_{i j, k}$ and $Q_{i j}$ are smooth functions on $\left.B M\right|_{\bar{W}}$.

Suppose that $n=1$. Recall that $D_{R}\left(\pi_{R} \phi\right)$ is defined as

$$
D_{R} \pi_{R} \phi:=d(\phi+f \eta)=d \phi+d_{R} f \wedge \eta+f \wedge d \eta .
$$

where $f \in \Gamma^{s-1}(M)$ is the unique function with $\eta \wedge(d \phi+f d \eta)=0$. Because the map $h \mapsto h \eta \wedge d \eta$ is a smooth linear isomorphism, $\|f\|_{s-1} \prec\|f \eta \wedge d \eta\|_{s-1}$. We can, therefore, estimate as follows:

$$
\left\|D_{R} \pi_{R} \phi\right\|_{s-2} \prec\|d \phi\|_{s-2}+\|f\|_{s-1} \prec\|d \phi\|_{s-2}+\|d \phi \wedge \eta\|_{s-1} .
$$

Thus, we need only to estimate $\|d \phi\|_{s-2}$ and $\|d \phi \wedge \eta\|_{s-1}$ :

$$
\begin{aligned}
\|d \phi\|_{W, s-2} & =\left\|d\left(Q_{i j, k}(x, X) X^{i} X^{j} d x^{k}+Q_{i j}(x, X) X^{i} d X^{j}\right)\right\|_{W, s-2} \\
& \prec\|X\|_{W, s-1}\|X\|_{W, s}, \\
\|(d \phi \wedge \eta)\|_{W, s-1} & \prec\left\|d\left(Q_{i j, k}(x, X) X^{i} X^{j} d x^{k}+Q_{i j}(x, X) X^{i} d X^{j}\right) \wedge \eta\right\|_{W, s-1} \\
& \prec\|X\|_{W, s-1}\|X\|_{W, s} .
\end{aligned}
$$

The proof of the second inequality follows by similar reasoning.

Now suppose that $n>1$. Then

$$
\begin{aligned}
& \left\|d_{R} \pi_{R}\left(F_{X}^{*} \eta-\eta-\mathcal{L}_{X}\right)\right\|_{W, s-1} \\
& \quad=\left\|\pi_{R} d \phi\right\|_{W, s-1} \\
& \left.\quad=\| d_{R}\left(Q_{i j, k}(x, X) X^{i} X^{j} d x^{k}\right)+d_{R}\left(Q_{i j}(x, X) X^{i}\right) \wedge d_{R} X^{j}\right) \|_{W, s-1} \\
& \quad \prec\|X\|_{W, s}\|X\|_{W, s-1} .
\end{aligned}
$$

The proof of the last inequality in the statement of the lemma follows by similar reasoning.

Proof of Proposition 4.6.1. Recall the definition of $\Psi$,

$$
\Psi(X)-X=\Phi^{-1} \circ \pi \circ \Phi(X)-X=\Phi^{-1} \circ \pi \circ \Phi(X)-\Phi^{-1} \circ \Phi(X),
$$

where $\Psi$ is the map defined in Theorem 4.3.7 and where $\pi(g \oplus \alpha \oplus \omega)=g$. Because $\Phi^{-1}$ is smooth (and thus of class $C^{1}$ ), equation (4.6.4) implies the inequality

$$
\|\Psi(X)-X\|_{s} \prec\|\| \pi \circ \Phi(X)-\Phi(X)\|\|_{s}=\left\|\pi^{\perp} \Phi(X)\right\|_{s},
$$

where $\pi^{\perp}(g \oplus \alpha \oplus \omega):=\alpha \oplus \omega$ and $\||| g \oplus \alpha \oplus \omega \mid\|_{s}:=\|g\|_{s+1}+\|\alpha \oplus \omega\|_{s}$. Consequently, to prove the estimate (i), we need only to estimate the terms 
in the expansion

$$
\pi^{\perp} \Phi(X)=\left\{\begin{array}{cc}
(n+1) G_{R} \delta_{R}\left(\pi_{R} F_{X}^{*} \eta\right) & \\
\oplus\left(G_{R} D_{R}^{*} D_{R}\left(\pi_{R} F_{X}^{*} \eta\right) \oplus H_{R}\left(\pi_{R} F_{X}^{*} \eta\right)\right), & \text { for } n=1, \\
(n+1) G_{R} \delta_{R}\left(\pi_{R} F_{X}^{*} \eta\right) & \\
\oplus\left(n G_{R} \delta_{R} d_{R}\left(\pi_{R} F_{X}^{*} \eta\right) \oplus H_{R}\left(\pi_{R} F_{X}^{*} \eta\right)\right), & \text { for } n>1
\end{array}\right.
$$

given in Theorem 4.3.7. By Lemma 4.2.7 we have the local formula

$$
F_{X}^{*} \eta=\eta+\mathcal{L}_{X} \eta+Q_{i j}^{1}(x, X) X^{i} X^{j}+Q_{i j}^{2}(x, X) X^{i} d X^{j} .
$$

Therefore, because $X$ is contact and so $\pi_{R}\left(\mathcal{L}_{X} \eta\right)=0$, Lemma 4.6 .2 implies the estimate

$\left\|\pi_{R}\left(F_{X}^{*} \eta\right)\right\|_{s-1} \prec\left\|\pi_{R} \eta\right\|_{s-1}+\left\|\pi_{R}\left(\mathcal{L}_{X} \eta\right)\right\|_{s-1}+\|X\|_{s}\|X\|_{s-1}=\|X\|_{s}\|X\|_{s-1}$.

Taking into account the orders of the various operators in equation (4.6.5) and recalling Lemma 4.6.3, we can estimate as follows:

For $n \geq 1$,

$$
\begin{aligned}
& \left\|G_{R} \delta_{R} \pi_{R}\left(F_{X}^{*} \eta\right)\right\|_{s} \prec\left\|\pi_{R}\left(F_{X}^{*} \eta\right)\right\|_{s-1} \prec\|X\|_{s}\|X\|_{s-1} \\
& \left\|H_{R} \pi_{R}\left(F_{X}^{*} \eta\right)\right\|_{s} \prec\left\|\pi_{R}\left(F_{X}^{*} \eta\right)\right\|_{s-1} \prec\|X\|_{s}\|X\|_{s-1} \text {; }
\end{aligned}
$$

for $n=1$,

$$
\left\|G_{R} D_{R}^{*} D_{R} \pi_{R}\left(F_{X}^{*} \eta\right)\right\|_{s} \prec\left\|D_{R} \pi_{R}\left(F_{X}^{*} \eta\right)\right\|_{s-2} \prec\|X\|_{s}\|X\|_{s-1} ;
$$

and for $n>1$,

$$
\left\|G_{R} \delta_{R} d_{R} \pi_{R}\left(F_{X}^{*} \eta\right)\right\|_{s} \prec\left\|d_{R} \pi_{R}\left(F_{X}^{*} \eta\right)\right\|_{s-1} \prec\|X\|_{s}\|X\|_{s-1} .
$$

This concludes the proof of (i).

We now show that

$$
\begin{aligned}
\left\|\left(\Psi\left(X_{2}\right)-X_{2}\right)-\left(\Psi\left(X_{1}\right)-X_{1}\right)\right\|_{s} \prec & \left\|X_{2}-X_{1}\right\|_{s-1}\left(\left\|X_{2}\right\|_{s}+\left\|X_{1}\right\|_{s}\right) \\
& +\left\|X_{2}-X_{1}\right\|_{s}\left(\left\|X_{2}\right\|_{s-1}+\left\|X_{1}\right\|_{s-1}\right) .
\end{aligned}
$$

Set $Y_{i}:=\Psi\left(X_{i}\right)-X_{i}, i=1,2$. Differentiability of the map $\Phi^{-1}$ implies the inequality

$$
\left\|Y_{2}-Y_{1}\right\|_{s}=\left\|\left(\Psi\left(X_{2}\right)-X_{2}\right)-\left(\Psi\left(X_{1}\right)-X_{1}\right)\right\|_{s} \prec\left\|\left|\Phi\left(Y_{2}-Y_{1}\right) \|\right|_{s} ;\right.
$$

which, by adding and subtracting a term and applying the triangle inequality, implies the inequality

$$
\begin{aligned}
\left\|Y_{2}-Y_{1}\right\|_{s} \prec & \|\|\left(\pi^{\perp} \circ \Phi\left(X_{2}\right)-\pi^{\perp} \circ \Phi\left(X_{1}\right)\right)\|\|_{s} \\
& +\left\||| \Phi\left(Y_{2}-Y_{1}\right)+\left(\pi^{\perp} \circ \Phi\left(X_{2}\right)-\pi^{\perp} \circ \Phi\left(X_{1}\right)\right)\right\| \|_{s} .
\end{aligned}
$$

To conclude the proof, we need only to estimate each term on the right-hand side of (4.6.6). 
To estimate the first term, note that by Lemma 4.2.7 applied to the contact vector fields $X_{i}$, we have the local formula

$$
\pi_{R} F_{X_{i}}^{*} \eta=\pi_{R}\left(\eta+\mathcal{L}_{X_{i}} \eta+Q_{\eta}\left(X_{i}\right)\right)=\pi_{R}\left(Q_{\eta}\left(X_{i}\right)\right) .
$$

This and equation (4.6.5) imply the inequalities

$$
\begin{aligned}
\| \mid \pi^{\perp} & \circ \Phi\left(X_{2}\right)-\pi^{\perp} \circ \Phi\left(X_{1}\right) \|_{s} \\
\prec & \left\|\pi_{R}\left(Q_{\eta}\left(X_{2}\right)-Q_{\eta}\left(X_{1}\right)\right)\right\|_{s-1} \\
& \quad+\left\|D_{R} \pi_{R}\left(Q_{\eta}\left(X_{2}\right)-Q_{\eta}\left(X_{1}\right)\right)\right\|_{s-2} \text { for } n=1
\end{aligned}
$$

and

$$
\begin{aligned}
& \prec\left\|\pi_{R}\left(Q_{\eta}\left(X_{2}\right)-Q_{\eta}\left(X_{1}\right)\right)\right\|_{s-1} \\
& \quad+\left\|d_{R} \pi_{R}\left(Q_{\eta}\left(X_{2}\right)-Q_{\eta}\left(X_{1}\right)\right)\right\|_{s-1} \text { for } n>1 .
\end{aligned}
$$

Evoking Lemmas 4.6.2 and 4.6.3 then yields the estimate of the first term we need:

$$
\begin{aligned}
\left\|\mid\left(\pi^{\perp} \circ \Phi\left(X_{2}\right)-\pi^{\perp} \circ \Phi\left(X_{1}\right)\right)\right\| \|_{s} \prec & \left\|\left(X_{2}-X_{1}\right)\right\|_{s-1}\left(\left\|X_{2}\right\|_{s}+\left\|X_{1}\right\|_{s}\right) \\
& +\left\|\left(X_{2}-X_{1}\right)\right\|_{s}\left(\left\|X_{2}\right\|_{s-1}+\left\|X_{1}\right\|_{s-1}\right) .
\end{aligned}
$$

We estimate the second term in (4.6.6) as follows. We first claim that $\Phi\left(Y_{2}-Y_{1}\right)=\pi^{\perp} \circ \Phi\left(Y_{2}-Y_{1}\right)$. To see this, use the definition $\Psi(X)=$ $\Phi^{-1} \circ \pi \circ \Phi(X)$ for any vector field $X$ and the linearity of the map $\pi \circ \Phi$ to write $\pi \circ \Phi\left(Y_{i}\right)$ in the form

$$
\pi \circ \Phi\left(Y_{i}\right)=\pi \circ \Phi\left(\Psi\left(X_{i}\right)-X_{i}\right)=\pi \circ \Phi\left(\Phi^{-1} \circ \pi \circ \Phi\left(X_{i}\right)\right)-\pi \circ \Phi\left(X_{i}\right)=0 .
$$

It follows that $\pi \circ \Phi\left(Y_{2}-Y_{1}\right)=0$; hence, $\pi^{\perp} \circ \Phi\left(Y_{2}-Y_{1}\right)=\Phi\left(Y_{2}-Y_{1}\right)$. Next, since $\Psi(X)=\Phi^{-1} \circ \pi \circ \Phi(X)$, we have

$$
\pi^{\perp} \circ \Phi \circ \Psi\left(X_{i}\right)=\pi^{\perp} \pi \circ \Phi\left(X_{i}\right)=0 .
$$

Combining these two identities shows the second term in (4.6.6) can be written

$$
\begin{aligned}
& \Phi\left(Y_{2}-Y_{1}\right)+\left(\pi^{\perp} \circ \Phi\left(X_{2}\right)-\pi^{\perp} \circ \Phi\left(X_{1}\right)\right) \\
& \quad=\pi^{\perp}\left\{\Phi\left(Y_{2}-Y_{1}\right)+\left(\Phi\left(X_{2}\right)-\Phi\left(\Psi\left(X_{2}\right)\right)-\Phi\left(X_{1}\right)+\Phi\left(\Psi\left(X_{1}\right)\right)\right\} .\right.
\end{aligned}
$$

We now show that

$$
\begin{aligned}
& \pi^{\perp}\left\{\Phi\left(Y_{2}-Y_{1}\right)+\left(\Phi\left(X_{2}\right)-\Phi\left(\Psi\left(X_{2}\right)\right)-\Phi\left(X_{1}\right)+\Phi\left(\Psi\left(X_{1}\right)\right)\right\}\right. \\
& \quad=L\left(Q_{\eta}\left(Y_{2}-Y_{1}\right)-\left(Q_{\eta}\left(\Psi\left(X_{2}\right)\right)-Q_{\eta}\left(\Psi\left(X_{1}\right)\right)\right)+\left(Q_{\eta}\left(X_{2}\right)-Q_{\eta}\left(X_{1}\right)\right)\right),
\end{aligned}
$$

where $L$ is the linear operator defined by

$$
L(\phi):= \begin{cases}\left\{(n+1) G_{R} \delta_{R} \oplus\left(G_{R} D_{R}^{*} D_{R}+H_{R}\right)\right\} \circ \pi_{R}(\phi), & \text { for } n=1, \\ \left\{(n+1) G_{R} \delta_{R} \oplus\left(n G_{R} \delta_{R} d_{R}+H_{R}\right)\right\} \circ \pi_{R}(\phi), & \text { for } n>1 .\end{cases}
$$


To see this, apply Lemma 4.2 .7 with $\phi=\eta$ to the vector fields $Y_{2}-Y_{1}, X_{i}$, and $\Psi\left(X_{i}\right)$ to obtain the three expansions

$$
\begin{aligned}
F_{X_{i}}^{*} \eta & =\eta+\mathcal{L}_{X_{i}} \eta+Q_{\eta}\left(X_{i}\right), \\
F_{\Psi\left(X_{i}\right)}^{*} \eta & =\eta+\mathcal{L}_{\Psi\left(X_{i}\right)} \eta+Q_{\eta}\left(\Psi\left(X_{i}\right)\right), \\
F_{\left(Y_{2}-Y_{1}\right)}^{*} \eta & =\mathcal{L}_{Y_{2}} \eta-\mathcal{L}_{Y_{1}} \eta+Q_{\eta}\left(Y_{2}-Y_{1}\right) .
\end{aligned}
$$

Substituting these expressions into Equation (4.6.5) and collecting terms reveals that the terms involving the Lie derivative of $\eta$ cancel to yield the identity (4.6.7). Thus, by the triangle inequality, to estimate the second term in (4.6.6), we need only to estimate each term in the sum

$$
\begin{aligned}
& \left\|\left|L\left(Q_{\eta}\left(Y_{2}-Y_{1}\right)\right)\right|\right\|_{s}+\left\||| L\left(Q_{\eta}\left(\Psi\left(X_{2}\right)\right)-Q_{\eta}\left(\Psi\left(X_{1}\right)\right)\right)\right\|_{s} \\
& \quad+\left\|L\left(Q_{\eta}\left(X_{2}\right)-Q_{\eta}\left(X_{1}\right)\right)\right\|_{s} .
\end{aligned}
$$

For each term, we use the estimates

$$
\|L(\phi)\| \|_{s} \prec \begin{cases}\left\|\pi_{R} \phi\right\|_{s-1}+\left\|D_{R} \pi_{R} \phi\right\|_{s-2}+\left\|H_{R} \pi_{R} \phi\right\|_{s}, & \text { for } n=1, \\ \left\|\pi_{R} \phi\right\|_{s-1}+\left\|d_{R} \pi_{R} \phi\right\|_{s-1}+\left\|H_{R} \pi_{R} \phi\right\|_{s}, & \text { for } n>1 .\end{cases}
$$

Noting the degree of the various linear operators in the definition of $L$ and employing Lemmas 4.6.2 and 4.6.3 shows that

$$
\begin{aligned}
\left\|L\left(Q_{\eta}\left(Y_{2}-Y_{1}\right)\right)\right\|_{s} \prec & \left(\left\|Y_{2}-Y_{1}\right\|_{s}\right)\left\|Y_{2}-Y_{1}\right\|_{s-1}, \\
\|\| L\left(Q_{\eta}\left(\Psi\left(X_{2}\right)\right)-Q_{\eta}\left(\Psi\left(X_{1}\right)\right)\right) \|_{s} \prec & \left(\left\|\Psi\left(X_{1}\right)\right\|_{s}+\left\|\Psi\left(X_{2}\right)\right\|_{s}\right) \cdot \| \Psi\left(X_{2}\right) \\
& -\Psi\left(X_{1}\right) \|_{s-1}+\left(\left\|\Psi\left(X_{1}\right)\right\|_{s-1}\right. \\
& \left.+\left\|\Psi\left(X_{2}\right)\right\|_{s-1}\right) \cdot\left\|\Psi\left(X_{2}\right)-\Psi\left(X_{1}\right)\right\|_{s},
\end{aligned}
$$

and

$$
\begin{aligned}
\left\|L L\left(Q_{\eta}\left(X_{2}\right)-Q_{\eta}\left(X_{1}\right)\right)\right\| \|_{s} \prec & \left(\left\|X_{1}\right\|_{s}+\left\|X_{2}\right\|_{s}\right) \cdot\left\|X_{2}-X_{1}\right\|_{s-1} \\
& +\left(\left\|X_{1}\right\|_{s-1}+\left\|X_{2}\right\|_{s-1}\right) \cdot\left\|X_{2}-X_{1}\right\|_{s} .
\end{aligned}
$$

To conclude the estimate of (4.6.6), note that since $\Psi$ is $C^{1}$, we can replace $\Psi\left(X_{i}\right)$ by $X_{i}$ and $Y_{2}-Y_{1}$ by $X_{2}-X_{1}$ everywhere in the first two of the previous three inequalities.

\section{References}

[ABE] S. Anco, J. Bland and M. Eastwood, Some Penrose transforms in complex differential geometry, Sci. China Ser. A 49 (2006), 1599-1610.

[Arn] V.I. Arnold, Mathematical methods of classical mechanics, Springer-Verlag, New York, Heidelberg, Berlin, 1978.

[B] J. Bland, Contact geometry and CR-structures on $S^{3}$, Acta Math. 172 (1994), $1-49$. 
[BD1] J. Bland and T. Duchamp, Deformation theory for the hyperplane line bundle on $\mathbf{P}^{1}$, in 'CR-Geometry and Overdetermined Systems (Osaka 1994)', Adv. Stud. Pure Mathematics 25, Math. Soc. Japan, Tokyo, 1997, 41-59.

[BD2] J. Bland and T. Duchamp, Anisotropic Estimates for Sub-elliptic Operators, Science in China, Ser. A: Mathematics 51, (Science Press/Springer-Verlag), 2008, 509-522.

[BD3] J. Bland and T. Duchamp, The space of Cauchy-Riemann structures on 3-D compact contact manifolds, J. Diff. Geom. 88 (2011), 189-230.

[BlEp] J. Bland and C.L. Epstein, Embeddable CR-structures and deformations of pseudoconvex surfaces, Part I: Formal deformations, J. Algebra Geom. 5 (1996), $277-368$.

[Bi] O. Biquard, Metriques autoduales sur la boule, Invent. Math. 148 (2002), 545-607.

[CL] J.-H. Cheng and J.M. Lee, A local slice theorem for 3-dimensional CR structures, Amer. J. Math. 117 (1995), 1249-1298.

[E] D. Ebin, The manifold of Riemannian metrics, in 'Global Analysis', eds. S.S. Chern and S. Smale, Proc. Symp. in Pure Mathematics, Amer. Math. Soc. XV, 1970, 11-40.

[EM] D. Ebin and J. Marsden, Groups of diffeomorphisms and the motion of an incompressible fluid, Ann. Math. 92 (1970), 102-163.

[EH] C.L. Epstein and G. Henkin, Extension of CR-structures for 3-dimensional pseudoconcave manifolds, in 'Multidimensional Complex Analysis and Partial Differential Equations', Contemp. Math., 205, Amer. Math. Soc. 1997, 51-67.

[FS] G.B. Folland and E.M. Stein, Estimates for the $\bar{\partial}_{b}$ complex and analysis on the Heisenberg group, Commun. Pure Appl. Math. 27 (1974), 429-522.

[G] J. Gray, Some global properties of contact structures, Ann. Math. 69 (1959), 421-450.

[Ham] R. Hamilton, The inverse function theorem of Nash and Moser, Bull. AMS 7 (1982), 65-222.

[Kir] G.K. Kiremidjian, A direct extension method for CR structures, Math. Ann. 242 (1979), 1-19.

[Ku] M. Kuranishi, Application of $\bar{\partial}_{b}$ to deformation of isolated singularities, Proc. Symp. Pure Mathematics, 30 (1977), 97-106.

[O1] H. Omori, On the group of diffeomorphisms on a compact manifold, in 'Global Analysis', eds. S.S. Chern and S. Smale, Proc. Symp. Pure Mathematics, Amer. Math. Soc., Vol. XV, 1970, 167-183.

[O2] H. Omori, Infinite dimensional lie transformations groups, Lecture Notes in Mathematics, 427, Springer-Verlag, Berlin, Heidelberg, New York, 1974.

[Miy1] K. Miyajima, CR construction of the flat deformations of normal isolated singularities, J. Algebra Geom. 8 (1999), 403-470.

[Miy2] K. Miyajima, A note on the closed rangeness of vector bundle valued tangential Cauchy-Riemann operator, in "Analysis and Geometry in Several Complex Variables", eds. G. Komatsu and M. Kuranishi, Trends in Mathematics, Birkhauser, Boston, 1999, 193-208.

[Pal] R. Palais, Foundations of Global non-linear analysis, W.A. Benjamin, Inc., New York, 1968. 
[R] M. Rumin, Formes differentielles sur les varietes de contact, J. Diff. Geom. 39 (1994), 281-330.

UnIVERSITY OF TORONTO

E-mail address: bland@math.toronto.edu

UNIVERSITY OF WASHINGTON

E-mail address: duchamp@math.washington.edu

Received 05/10/2011, accepted 02/12/2013

John Bland was partially supported by an NSERC grant. Tom Duchamp was partially supported by an NSF grant. 